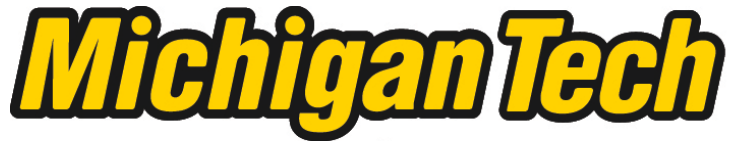 \\ Michigan Technological University Create the Future Digital Commons @ Michigan Tech
}

Dissertations, Master's Theses and Master's Reports - Open

Dissertations, Master's Theses and Master's

Reports

2006

Optimal beam forming for laser beam propagation through random media

Baoyong Liu

Michigan Technological University

Follow this and additional works at: https://digitalcommons.mtu.edu/etds

Part of the Electrical and Computer Engineering Commons

Copyright 2006 Baoyong Liu

\section{Recommended Citation}

Liu, Baoyong, "Optimal beam forming for laser beam propagation through random media", Dissertation, Michigan Technological University, 2006.

https://doi.org/10.37099/mtu.dc.etds/78

Follow this and additional works at: https://digitalcommons.mtu.edu/etds

Part of the Electrical and Computer Engineering Commons 


\title{
OPTIMAL BEAM FORMING FOR LASER BEAM PROPAGATION THROUGH RANDOM MEDIA
}

By

BAOYONG LIU

\author{
A DISSERTATION \\ Submitted in partial fulfillment of the requirements \\ for the degree of \\ DOCTOR OF PHILOSOPHY \\ (Electrical and Computer Engineering) \\ MICHIGAN TECHNOLOGICAL UNIVERSITY \\ 2006 \\ Copyright (C) Baoyong Liu 2006
}


This dissertation, "Optimal Beam Forming for Laser Beam Propagation through Random Media," is hereby approved in partial fulfillment of the requirements for the degree of DOCTOR OF PHILOSOPHY in the field of Electrical and Computer Engineering.

DEPARTMENT:

Electrical and Computer Engineering

Signatures:

Dissertation Advisor

Timothy J. Schulz

Committee

Michael C. Roggemann

Jeffrey B. Burl

Mark S. Gockenbach

Department Chair

Timothy J. Schulz

Date 
To My Parents 


\begin{abstract}
Focusing optical beams on a target through random propagation media is very important in many applications such as free space optical communications and laser weapons. Random media effects such as beam spread and scintillation can degrade the optical system's performance severely. Compensation schemes are needed in these applications to overcome these random media effects. In this research, we investigated the optimal beams for two different optimization criteria: one is to maximize the concentrated received intensity and the other is to minimize the scintillation index at the target plane. In the study of the optimal beam to maximize the weighted integrated intensity, we derive a similarity relationship between pupil-plane phase screen and extended Huygens-Fresnel model, and demonstrate the limited utility of maximizing the average integrated intensity. In the study of the optimal beam to minimize the scintillation index, we derive the firstand second-order moments for the integrated intensity of multiple coherent modes. Hermite-Gaussian and Laguerre-Gaussian modes are used as the coherent modes to synthesize an optimal partially coherent beam. The optimal beams demonstrate evident reduction of scintillation index, and prove to be insensitive to the aperture averaging effect.
\end{abstract}




\section{Acknowledgement}

First and foremost, I would like to thank my advisor Dr. Timothy Schulz, for his support, guidance, and encouragement in the Ph.D program. He showed me the way to approach research problems and taught me how to express my ideas. I am deeply impressed by his inspiring and encouraging way to guide me to a deeper understanding of knowledge. I am grateful for his advise and comments in this dissertation.

I also would like to thank the rest of my thesis committee: Dr. Michael Roggemann, Dr. Jeffrey Burl, and Dr. Mark Gockenbach, for their kind help and valuable comments on this work. Thanks also go to my friends and colleagues, who give me help and have fun with me. I really enjoy the life with them in the beautiful Keweenaw Peninsula.

Last, I wish to thank my parents, my sister, and my brother, for their continued support and encouragement. I am also very grateful to my wife Ling, for her love and patience during the Ph.D period. 


\section{Contents}

Abstract iv

Acknowledgement $\quad$ V

List of Figures xiv

List of Tables $\quad$ xvi

1 Introduction 1

1.1 Motivation . . . . . . . . . . . . . . . . 1

1.2 Research Goals . . . . . . . . . . . . . . . . 5

1.3 Dissertation Organization . . . . . . . . . . . . 7

2 Background $\quad 8$

2.1 Reasons for Optical Disturbance in Random Medium . . . . . 8

2.2 Waves in Random Media . . . . . . . . . . . . . . . . 11

2.3 Turbulence Effects on Beam Propagation . . . . . . . . . . . . 12

2.4 Adaptive Optics . . . . . . . . . . . . . . . . . . . . 14

3 Mathematical Models $\quad 16$

3.1 Scalar Diffraction in Homogeneous Medium . . . . . . . . 16

3.2 Optical Beams . . . . . . . . . . . . . . . . . 19 
3.2.1 Paraxial waves and Gaussian beam . . . . . . . . . . 19

3.2.2 Hermite-Gaussian beams . . . . . . . . . . . . . . 21

3.2.3 Laguerre-Gaussian beams . . . . . . . . . . . . 22

3.3 Optical Coherence . . . . . . . . . . . . . 25

3.3.1 Mutual coherence function . . . . . . . . . . . 25

3.3.2 Propagation of coherence . . . . . . . . . . . . . 28

3.3.3 Coherent mode representation . . . . . . . . . . . 29

3.4 Statistical Model of Atmospheric Turbulence . . . . . . . . . . 30

3.4.1 Kolmogorov turbulence model . . . . . . . . . . . . . 31

3.4.2 Power spectrum of refractive index fluctuations . . . . 32

3.5 Wave Propagation through Turbulence . . . . . . . . . . . 34

3.5.1 Inhomogeneous wave equation . . . . . . . . . . 34

3.5.2 Born approximation . . . . . . . . . . . . 36

3.5.3 Rytov approximation . . . . . . . . . . . 37

3.5.4 Extended Huygens-Fresnel principle . . . . . . . . . . . 39

3.5.5 Turbulence layer model . . . . . . . . . . . . . . . . . . 41

3.6 Performance Metrics _. . . . . . . . . . . . . . . . . . . . . 42

3.6.1 Maximization of the integrated intensity . . . . . . 43

3.6.2 Minimization of the scintillation index . . . . . . . 43

4 Optimal Beam to Maximize the Integrated Intensity 45

4.1 Optimal Beam in Free Space . . . . . . . . . . . . . . . . 49

4.2 Optimal Beam to Maximize the Average Integrated Intensity . 50

4.3 Averaged Kernel in Turbulence . . . . . . . . . . . . . . . 52 
4.3.1 Averaged kernel for the phase screen model . . . . . . 52

4.3.2 Averaged kernel for the extended Huygens-Fresnel principle model . . . . . . . . . . . . . . . . 56

4.4 Performance of the Optimal Beams . . . . . . . . . . . . 60

4.4.1 One dimensional experiment . . . . . . . . . . . 61

4.4.2 Two dimensional experiment . . . . . . . . . . . 64

4.4 .3 Performance analysis . . . . . . . . . . . . . . . 64

5 Optimal Beam to Minimize the Scintillation Index $\quad 67$

5.1 Optimal Pupil Plane Mutual Coherence . . . . . . . . . . . . 68

5.2 Optimal Beams in Random Phase Screen Model . . . . . . . . 71

5.2.1 First moment of the integrated intensity . . . . . . 72

5.2.2 Second moment of the integrated intensity . . . . . . 72

5.3 Optimal Beams in the extended Huygens-Fresnel principle model 73

5.3.1 First moment of the integrated intensity . . . . . . . 74

5.3.2 Second moment of the integrated intensity . . . . . 75

5.4 Selection of coherent modes . . . . . . . . . . . 77

5.4.1 Scintillation reduction by using Hermite-Gaussian beams 78

5.4.2 Scintillation reduction by using Laguerre-Gaussian beams 81

5.5 Monte-Carlo Simulation . . . . . . . . . . . . . . 85

5.5.1 Phase screen generation . . . . . . . . 86

5.5.2 Pupil plan phase screen simulation . . . . . . . 88

5.5.3 Multiple phase screen simulation . . . . . . . . . 92

5.6 Aperture Averaging of Scintillation . . . . . . . . . . . 94 
6 Summary

6.1 Conclusions . . . . . . . . . . . . . . . . . . . . . . . . 99

6.2 Future work . . . . . . . . . . . . . . . . . 101

$\begin{array}{ll}\text { Appendices } & 102\end{array}$

$\begin{array}{ll}\text { A Glossary of Symbols } & 102\end{array}$

$\begin{array}{ll}\text { Bibliography } & 105\end{array}$ 


\section{List of Figures}

3.1 The scalar diffraction of optical wave from the aperture plane to the target plane. . . . . . . . . . . . . 17

3.2 Hermite Gaussian beams $f_{l, m}(x, y)$ with $l=0,1,2,3$ for columns from left to right and $m=0,1,2,3$ for rows from top to bottom . 23

3.3 Laguerre Gaussian beams $f_{p, m}(r, \theta)$ with $p=0,1,2,3$ for rows from top to bottom and $m=0,1,2,3$ for columns from left to right. . . . . . . . . . . . . . . . 24

3.4 Propagation of optical coherence. . . . . . . . . . . . . 28

3.5 Turbulence layer propagation model: (a) Pupil plane phase screen model. (b) Multiple phase screen model. . . . . . . . . 42

4.1 Free space diffraction of focused field from circular aperture: $\lambda=10^{-6}, d=10^{4}, D=0.2$, all units in meters. (a) Transmitting aperture $a(\vec{u})$. (b) Normalized amplitude of the received field $g(\vec{v}) . \ldots \ldots \ldots \ldots \ldots \ldots$ 
4.2 Airy pattern: (a)Normalized Airy pattern slice, (b)Fractional integrated intensity $\mathcal{I} / \mathcal{I}_{0}$ vs. normalized target plane radius $(r D) /(\lambda d) \ldots \ldots \ldots \ldots \ldots \ldots \ldots$

4.3 Kernel $\mathcal{H}_{0}\left(u, u^{\prime}\right)$ for one dimensional propagation in free space with rectangular weighting function. . . . . . . . . 51

4.4 Averaged kernel $\left\langle\mathcal{H}\left(u, u^{\prime}\right)\right\rangle$ for one dimensional propagation through turbulence with rectangular weighting function and $r_{0}=10 \mathrm{~cm} \ldots \ldots \ldots \ldots \ldots \ldots \ldots$

4.5 Weighting function changes: (a) Original weighting function. (b) New weighting function in turbulence. . . . . . . . . . 56

4.6 Optimal beams and integrated intensity for one dimensional propagation in free space and random medium: (a) Optimal beam $\hat{f}(u)$ at transmitter aperture, (b) Fractional integrated intensity $\mathcal{I} / \mathcal{I}_{0}$ vs. receiver plane normalized radius $(r D) /(\lambda d) .63$

4.7 Optimal beams and integrated intensity for two dimensional propagation in free space and random medium: (a) A center slice of the optimal beam $\hat{f}(\vec{u})$ at transmitter aperture, (b) Fractional integrated intensity $\mathcal{I} / \mathcal{I}_{0}$ vs. receiver plane normalized radius $(r D) /(\lambda d)$ for focused beam and optimal beam. 65

5.1 Amplitude of one dimensional Hermite-Gaussian beams $f_{m}(x) .79$ 
5.2 Scintillation index for transmitting Hermite-Gaussian modes: (a) Scintillation index for single modes. (b) Reduction of the optimal scintillation index when the number of HermiteGaussian modes increased. . . . . . . . . . . . . . . 81

5.3 One dimensional Laguerre-Gaussian beams $f_{p, m}(x)$ with $m=0.83$

5.4 Scintillation index by transmitting Laguerre-Gaussian modes: (a) Scintillation index by transmitting single modes. (b) Reduction of the optimal scintillation index when the number of Laguerre-Gaussian modes increased . . . . . . . . . . . . . 84

5.5 Theoretical and Monte-Carlo simulation of the phase structure function $D_{\phi}(\Delta u)$ and $\left\langle\exp \left[\phi\left(u_{1}\right)-\phi\left(u_{2}\right)\right]\right\rangle$ of the random phase screen . . . . . . . . . . . . . . . 87

5.6 Monte-Carlo simulation by transmitting single one dimensional Hermite-Gaussian modes $f_{m}(x)$ : (a) Scintillation index, (b) average integrated intensity. . . . . . . . . . . . . 88

5.7 Monte-Carlo simulation by transmitting the optimal coherence composed of multiple one dimensional Hermite-Gaussian modes $f_{m}(x)$ : (a) Scintillation index, (b) average integrated intensity. . . . . . . . . . . . . . . . . 89

5.8 Monte-Carlo simulation by transmitting single one dimensional Laguerre-Gaussian modes $f_{m, 0}(x)$ : (a) Scintillation index, (b) average integrated intensity. . . . . . . . . . . . . 90 
5.9 Monte-Carlo simulation by transmitting the optimal coherence composed of multiple one dimensional Laguerre-Gaussian modes $f_{m, 0}(x)$ : (a) Scintillation index, (b) average integrated intensity. . . . . . . . . . . . . . . . 99

5.10 Monte-Carlo simulated performance of transmitting single two dimensional Hermite-Gaussian beam: (a) Scintillation index, (b) average integrated intensity. . . . . . . . . . . . . . 91

5.11 Monte-Carlo simulated performance of transmitting the optimal pupil plane coherence composed of multiple two dimensional Hermite-Gaussian beams: (a) Scintillation index, (b) average integrated intensity. . . . . . . . . . . . . . 92

5.12 Monte-Carlo simulated performance of transmitting single two dimensional Laguerre-Gaussian beams: (a) scintillation index, (b) average integrated intensity. . . . . . . . . . . . . 93

5.13 Monte-Carlo simulated performance of the optimal coherence composed of multiple two dimensional Laguerre-Gaussian modes: (a) scintillation index, (b) associated average integrated inten-

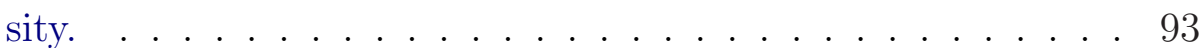

5.14 Monte-Carlo simulated performance of transmitting single two dimensional Hermite-Gaussian beams through multiple phase screens: (a) scintillation index, (b) average integrated intensity. 95 
5.15 Monte-Carlo simulated performance of transmitting optimal partial coherence synthesized by two dimensional HermiteGaussian beams through multiple phase screens: (a) scintillation index, (b) average integrated intensity. . . . . . . . . . . 95

5.16 Monte-Carlo simulated performance of transmitting single two dimensional Laguerre-Gaussian beams through multiple phase screens: (a) scintillation index, (b) average integrated intensity. 96

5.17 Monte-Carlo simulated performance of transmitting optimal partial coherence synthesized by two dimensional LaguerreGaussian beams through multiple phase screens: (a) scintillation index, (b) average integrated intensity. . . . . . . . . . 96

5.18 Aperture averaging effect in the pupil plane phase screen model: (a) Scintillation index vs. the normalized receiver radius for single Hermite Gaussian modes $f_{m}(x)$. (b) Optimal scintillation index vs. the normalized receiver radius for optimal beams synthesized by $N$ Hermite-Gaussian modes. . . . . . . . 98 


\section{List of Tables}

4.1 Physical parameters used in the one dimensional propagation simulation. . . . . . . . . . . . . . . 63

5.1 Propagation parameters used in the one dimensional simulation. 79

5.2 Fractional integrated intensity $\xi_{m}$ in free space, fractional average integrated intensity $\mu_{m}$ through turbulence, and the scintillation index $S_{m}$ of the received fields by transmitting single Hermite-Gaussian modes $f_{m}(u) \ldots \ldots$. . . . . . 80

5.3 Mutual intensity $\left\langle\mathcal{I}_{m} \mathcal{I}_{n}\right\rangle$ by using Hermite Gaussian beams. . 80

5.4 Optimal weighting $\alpha_{m}$, scintillation index $S_{I}$, and the fractional average integrated intensity $\mu$ when the first $N$ HermiteGaussian modes $f_{m}(x)$ are used to generate the optimal pupil plane partially coherence. . . . . . . . . . . . . . 82

5.5 Fractional integrated intensity $\xi_{p}$ in free space, fractional average integrated intensity $\mu_{p}$ through turbulence, and the scintillation index $S_{p}$ of the received fields by transmitting single Laguerre-Gaussian modes $f_{p, 0}(x) \ldots \ldots \ldots$. . . . . . 84 
5.6 Optimal weighting $\alpha_{p}$, scintillation index $S_{\mathcal{I}}$, and the fractional average integrated intensity $\mu$ when the first $N$ LaguerreGaussian modes $f_{p, 0}(u)$ are used to generate the optimal pupil plane partially coherence.

5.7 Mean square error $\epsilon^{2}$ of the Monte-Carlo simulation for different performance values: $\mu_{m}, S_{m}$ and $\left\langle\mathcal{I}_{m} \mathcal{I}_{n}\right\rangle \ldots \ldots$. . . . 89

5.8 Parameters used in the Monte-Carlo simulation of laser beams propagate through multiple random phase screens. . . . . . . . 94 


\section{Chapter 1}

\section{Introduction}

\subsection{Motivation}

Propagation of optical waves through random media such as the atmosphere, the ocean, and biological matter, is very important in many applications such as optical communications and astronomical imaging. In these situations, the temporally and spatially varying media change the amplitude and phase of the propagating optical fields in both time and space. These effects can cause attenuation or loss of the transmitted information and energy.

Because of their directionality and high energy concentration, laser beams are widely used in modern optical communications, remote sensing, and laser weapon systems. The performance of all these systems is limited by turbulence effects. Following is an introduction to some important applications of wave propagation through random medium. 


\section{Free space optical communications}

Because of the increasing need for bandwidth and high data rates, optical communication and network systems continue to be more and more important. Free space optical communications using laser beams have been developed for line-of-sight information transmission, such as in satellite communications. Systems with infrared, visible, and ultraviolet carrier frequencies are all included in optical communications. There are many advantages of optical communications compared with an RF system, such as wider bandwidth and higher power density in an extremely narrow beam [27]. But the detrimental effects of the propagation media form the main drawback of the system, because optical wavelengths are commensurate with molecule and particle sizes. Even in clear sky situations, laser beams are subject to turbulence, scattering and absorption through the atmosphere. In bad weather such as fog, rain and snow, the received optical power can be decreased and the bit error rate increased significantly.

\section{Laser radar system}

Laser radar, or lidar, is a short wave extension of radar, which uses radiation at wavelengths that are 10,000 to 100,000 times shorter than those used by conventional radar. Radiation scattered by a target is collected and processed to yield information about the target and the path to the target. By using higher frequency laser beams, higher accuracy and resolving power can be approached with lidar systems. Phase and amplitude fluctuations of the laser 
beam due to turbulent media can, however, disrupt lidar's functions such as target tracking, imaging and ranging.

\section{Astronomical imaging}

Astronomers have known for many years that the Earth's atmospheric propagation path causes phenomena such as image jitter and star twinkling. The phase distortions induced by propagation in turbulence cause degradation problems in imaging systems such as image blurring and image jitter which both contribute to a loss of resolution. This is the main reason that the Hubble Space Telescope was placed in space. Long exposure images from groundbased telescopes are severely blurred due to the much broader point spread function (PSF), a result of the average effects of turbulence. The Earth's turbulent atmosphere can also degrade radio astronomy systems. Although longer wavelength and different receivers (radio-telescopes) are used in radio astronomy, atmospheric turbulence has similar effects on optical and radio astronomy because both light and radio waves can be corrupted by random media.

\section{Laser guide star}

Reference beacons are needed in adaptive optics systems because wavefront compensation requires measurement of the turbulent propagation path. Two main requirements for the reference source are that it have enough brightness and it is located within the isoplanatic patch of the desired view angle [33]. Because there are few natural stars or objects that satisfy these requirements, 
man-made sources such as laser guide stars are developed to provide the required references. In the laser guide star system, high power laser beams are used to generate an artificial beacon in the sky by Rayleigh/Raman scattering or by sodium resonance scattering. Due to turbulent propagation paths, the generated laser beacon is subject to problems, such as broadening and reduced brightness.

\section{Remote sensing}

Remote sensing is now the principal technology used to observe, measure, and interpret an object without coming into direct contact with it. By utilizing sensing instruments, radiation of different wavelengths reflected or emitted from distant objects is detected and measured. Spatially distributed information about the object, such as spectral and physical properties, are acquired or measured within the sensing instrument. Laser beams are used in remote sensing to probe the properties of turbulent plasmas, hydrodynamic flows and the earth's atmosphere. Knowledge of meteorological parameters such as wind velocity and the strength of turbulence has become increasingly important in the study of optical waves propagation through atmospheric turbulence [66].

\section{Diffraction tomography}

Wave propagation through random media can also find applications in the three-dimensional reconstruction of absorbing and scattering objects. The accuracy of geometrical-radiation based computed tomography suffers from 
the effects of refraction and diffraction. When the sizes of inhomogeneities in the object become comparable to or smaller than the wavelength, it is not appropriate to use geometric propagation based ray theory. Tomographic reconstruction approaches based on Fourier diffraction theory are developed when the interaction of an object and a field is described by the inhomogeneous wave equation. This method is the so called diffraction tomography. In diffraction tomography, acoustic and electromagnetic waves do not travel along straight rays and the projections are not line integrals [40]. Diffraction and scattering of optical fields in the inhomogeneous medium must be involved.

\subsection{Research Goals}

It is well known that the propagation of waves in homogeneous media can be described by the wave equation, which is a second order linear differential equation. Optical fields in any position inside a homogeneous medium can be determined by the source fields in the aperture and the wave equation. However, for the problem of wave propagation in an inhomogeneous medium, there is no direct analytic solutions for the wave equation. In practice, approximate formalisms of the homogeneous medium wave propagation are widely used in the cases of weak inhomogeneities. Born and Rytov approximations are well known approximate methods [37]. Many other propagation models have been developed recently, including linear system representations and simulation schemes $[23,24]$. 
Historically, most of the original work on optical propagation deals with uniform plane or spherical waves. Recently, however, the propagation of other beams, such as truncated Gaussian beams, through random media have been widely studied [47]. But the problem of what type of optical beam profile will perform better through random media is seldom studied. By saying "perform better", we mean that the optical beam is less sensitive to turbulence effects such as beam spreading and scintillation. To be more precise, specific criteria are needed to evaluate the beam performance.

In this research, we will study the statistical properties of the optical beams after propagation through turbulent media. The effects of both source coherence and random media are investigated. The goal of this research is to compensate the random media effects in beam propagation by changing the transmitted optical fields or choosing the optical source coherence for certain performance metrics. Compensated beams are expected to concentrate more power in the target region or reduce the beam scintillation effectively.

In the study of the optimal beam to maximize the weighted integrated intensity, we derive a similarity relationship between pupil-plane phase screen and extended Huygens-Fresnel model, and demonstrate the limited utility of maximizing the average integrated intensity. In the study of the optimal beam to minimize the scintillation index, we derive the first- and second-order moments for the integrated intensity of multiple coherent modes. HermiteGaussian and Laguerre-Gaussian modes are used as the coherent modes to synthesize an optimal partially coherent beam. The optimal beams demon- 
strate evident reduction of scintillation index, and prove to be insensitive to the aperture averaging effect.

\subsection{Dissertation Organization}

The rest of this dissertation is organized as follows. In Chapter 2, I will describe the background of laser beam propagation through random media. Mathematic models of beam propagation, statistical random media, and optimization criterions are introduced in Chapter 3. The optimal beams to maximize the concentrated power and to minimize the scintillation index are investigated in Chapter 4 and Chapter 5 respectively. A summary and the conclusions of this research are given in Chapter 6 . 


\section{Chapter 2}

\section{Background}

Because of its importance in many applications, wave propagation through random media has been studied by many researchers $[73,37]$. In this chapter, we give an overview of the turbulence effects on beam propagation.

\subsection{Reasons for Optical Disturbance in Ran- dom Medium}

Random media may be grouped into three categories: random scatters, random continua, and rough surfaces [37]. Random scatters are a random distribution of many discrete scatters such as rain, fog, aerosols and blood cells. Random continuum refers to a medium whose index of refraction varies randomly and continuously in time and space, such as tropospheric and ionospheric turbulence, planetary atmospheres, solar corona, clear air turbulence and biological media.

The interaction of light with random media causes absorption, scattering, and distortions induced by random fluctuations of the refractive index. These 
are the most important causes of all types of random media effects in wave propagation and may happen all at once in one propagation case.

\section{Absorption}

When laser radiation is propagated in an absorbing medium, heating of the medium takes place and causes temperature gradients in the media. This effect will in turn cause media density changes and result in refractive index gradients being formed across the beam. These gradients act as a distributed lens which distorts the beam and decreases the peak intensity at the focal point [39]. This self-induced distortion and spreading of the beam is called thermal blooming, which is commonly met in high power laser beam propagation [70, 54].

\section{Scattering}

Scattering is the direction redistribution phenomenon caused by rough surface or small particles in the media. Rayleigh scattering, Mie scattering, and nonselective scattering are common situations associated with different sizes of the scatter.

The scattering effect of particles of matter was first studied by Lord Rayleigh, who studied the single scattering of a plane wave with a dielectric sphere in a vacuum and deduced that the color of the sky is the result of stronger scattering in the radiation of shorter wavelengths $[55,46]$. Rayleigh's scattering theory was extended by Mie, who considered light scattering by spheres of different sizes, comparable with the wavelength of light, 
and included the effect of absorption in his study [51]. Schuster studied the radiation in foggy atmospheres and initiated the radiative transport theory [37]. He is the first to consider multiple scattering, since single scattering theory becomes inadequate when the particle density is high.

\section{Turbulence}

Optical turbulence is a kind of random continuum with continually varying refractive index. People knew very early that star twinkling at night was caused by atmosphere turbulence, the density fluctuations caused by atmospheric temperature fluctuations. The induced random variations of the index-of-refraction produces phase distortion of the wavefront and continues to distort the amplitude for further propagation. Turbulence effects such as image blurring, laser beam spreading and scintillation can degrade the optical system performance severely.

Random optical turbulence can be described by its statistical properties, which are important in the study of wave propagation. Kolmogorov proposed a widely accepted model based on the mechanical structure of the turbulence $[44,45]$. From this turbulence model, power spectra of refractive index fluctuations are developed and modified with a wide range of usefulness in inhomogeneous wave propagation.

In this study, we will focus on the turbulence effects on optical beam propagation. It is assumed that atmospheric turbulence is a non-dissipative medium for the propagating wave, which means that absorption and ther- 
mal blooming can be ignored and there is no energy lost due to scattering turbulence eddies. Accordingly, optical effects arising out of absorption and scattering will not be discussed here. The approaches in this study treat the turbulence and its induced effects, such as the index of refractive fluctuations, as a continuum.

\subsection{Waves in Random Media}

The problem of wave propagation through random media has been studied by many researchers and a great deal of progress has been made in the past several decades $[12,73,72]$. Many propagation models and compensation schemes have been proposed and applied. Most of these schemes can be used in both image compensation and adaptive beam control.

Optical field propagation through turbulence can be described by the inhomogeneous wave equation with a randomly changing index of refraction. Because there is no analytic solution of the inhomogeneous wave equation, many approximate solutions are developed by using the small perturbation method. Earliest studies of this problem employed the geometric optics approximation [9], which is of very limited utility. A new method based on the Rytov approximation was developed by Tatarski in the late 1950's [73, 12] and has been adopted as the basic tool in this area by most researchers $[61,36]$.

The Rytov method has proved to agree with experimental data pretty well in weak turbulence cases. But in strong turbulence and long distance prop- 
agation cases, the experimental data does not match the predictions made using the Rytov approximation $[32,35]$. New theories has been developed for these situations, such as the diagrammatic techniques [11, 18, 17], transport methods [22], coherence theory [8, 52], Markov approximation [74] and several other approaches. Linear system representations of the inhomogeneous propagation were also developed [49].

The properties of wave propagation in random media and many important results obtained in recent history have been summarized in numerous reviews and books $[23,24,37,25,30,59,80,7,1,2]$.

\subsection{Turbulence Effects on Beam Propagation}

For laser beams propagating in turbulence, the cumulative effect of the refractive index fluctuation can cause significant wavefront and intensity distortions. Beam wander, beam spreading and scintillation are the main beam distortions resulting from turbulent media.

\section{Beam wander}

When the optical beam deviates from its original path, this is called beam wander. The phase front distortion induced by the random fluctuations in phase velocity alters the direction of beam propagation. The statistics of this angle of arrival fluctuations have been widely studied $[17,36]$. Measured data of beam wander under different conditions show that it is nearly independent of wavelength $[80,13,43]$. This random displacement of the beam spot 
centroid is caused by large eddies in the turbulence and the detected intensity for a detector appears to follow a Rayleigh distribution of probability in some cases [23].

\section{Beam spreading}

Beam spreading means that the optical beam size spreads beyond the dimensions predicted by diffraction theory, which is sometimes also called beam broadening. In the absence of turbulence, a laser beam exiting from an aperture of diameter $D$ would, in the far field, have an angular spread $\theta$ of order $\lambda / D$. The beam spreading is induced by small scale turbulence eddies in the propagation media and is usually wavelength dependent. Beam width or beam radius is commonly used as a measure of the beam spreading [19].

Beam spreading and beam wander are the beam counterparts to image blurring and image dancing. Beam wander is important for beam pointing and tracking considerations, while the short-time broadening is important for pulse propagation and high-energy laser systems [7].

\section{Scintillation}

Propagation of distorted wave fronts causes beam intensity fluctuations. Random deflection and interference between different portions of the wavefront lead to an internal breaking up of the beam spot into smaller "hot spots". This intensity fluctuation is described as scintillation, which includes both the temporal and spatial variations in received intensity within the receiver aperture. The scintillation phenomenon can be easily observed with 
the naked eye: bright stars close to the horizon twinkle strongly and change color on a time scale of seconds. The temporal frequency of the intensity fluctuations in atmospheric turbulence recorded at a fixed point within the beam usually varies between 1 and $100 \mathrm{~Hz}$.

Due to the importance of scintillation in optical detection, previous research has led to extensive theoretical calculations [73, 36] and experimental data $[42,20]$. Most of the theoretical studies are based on the Rytov method and use the scintillation index as a measurement. Then scintillation index is defined as the normalized variance of the intensity fluctuations, which is a function of the optical receiver size. As the diameter of the receiving aperture is increased, the magnitude of the fluctuation in the received power decreases. This is the so called aperture averaging effect which has been widely studied by many researchers $[26,43,34,14,3]$.

\subsection{Adaptive Optics}

Because turbulence effects severely degrade the system performance in applications such as imaging and optical communications, many compensation schemes based on adaptive optics have been proposed in recent history. The concept of adaptive optics was first proposed by Babcock in the 1950s as a possible method of compensating atmospheric turbulence in telescope imaging [4]. The core of adaptive optics is to measure the aberrations of an incoming wavefront and then cancel these in real time by introducing an electronically controlled phase shift. In the late 1960s, the first adaptive 
optics systems for laser beam control were developed to compensate for atmospheric turbulence in real time. As well as applications in astronomical imaging, adaptive optics has also been widely studied and used in retinal imaging and vision correction. Many surveys and reviews in this area have been summarized $[5,58,75,33]$.

There are three main components in a conventional adaptive optics system: wavefront sensing, electronic controller, and wavefront compensator. Turbulence properties can be measured from the distorted wavefront by using wavefront sensing devices such as the shear-interferometer and Hartmann wavefront sensors. According to this wavefront measurement, the controller sends out actuator positioning control signals to wavefront compensators such as deformable mirrors. The whole process is expected to perform at a higher rate than the rate of turbulence changing since this is a real time compensation.

Adaptive optics also have important applications in laser beam propagation through turbulence, such as the target-in-the-loop (TIL) problem. In TIL, laser beams are projected toward a target through random media and propagated back after surface scattering at the target. This problem has been involved in many applications such as laser target designation, remote sensing, and laser weapon systems [77]. In this dissertation, we will focus on this laser beam projection problem. 


\section{Chapter 3}

\section{Mathematical Models}

In this chapter, we describe the basic mathematic models that are used in this research for beam propagation, optical coherence, and atmosphere turbulence. Some terms and optimization metrics are also defined for beam performance measurement.

\subsection{Scalar Diffraction in Homogeneous Medium}

The scalar wave function $f(\vec{r}, t)$ propagating in a homogeneous medium must satisfy the scalar wave equation [10]

$$
\nabla^{2} f(\vec{r}, t)-\frac{1}{c^{2}} \frac{\partial^{2} f(\vec{r}, t)}{\partial t^{2}}=0
$$

where $\vec{r}=(x, y, z)$ is the position vector, $\nabla^{2}$ is the Laplacian operator:

$$
\nabla^{2}=\frac{\partial^{2}}{\partial x^{2}}+\frac{\partial^{2}}{\partial y^{2}}+\frac{\partial^{2}}{\partial z^{2}},
$$




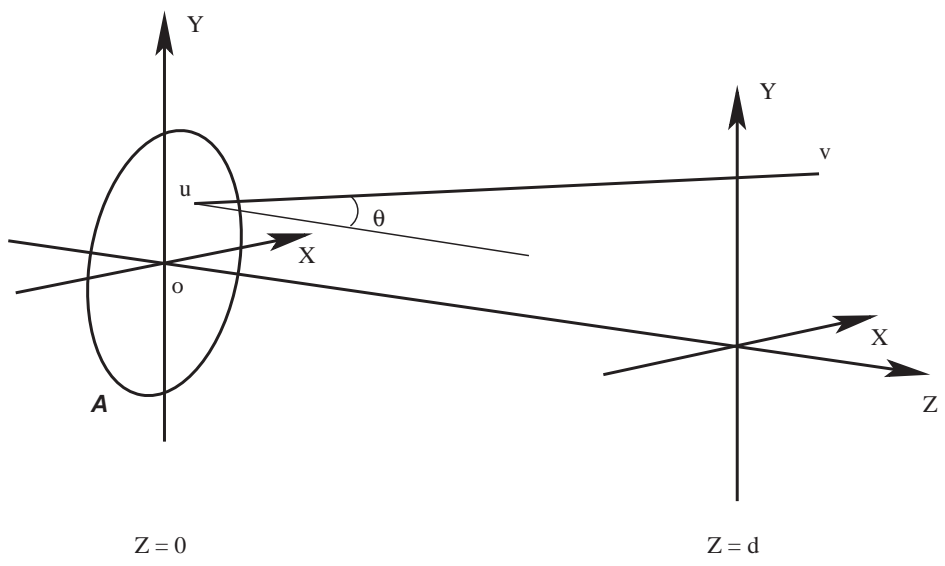

Figure 3.1: The scalar diffraction of optical wave from the aperture plane to the target plane.

and $c$ is the propagation speed of the wave in the medium. For monochromatic waves, the complex wave function $f(\vec{r}, t)$ can be written in the form

$$
f(\vec{r}, t)=f(\vec{r}) e^{j 2 \pi \nu t}
$$

where $f(\vec{r})$ is the complex amplitude and satisfies the Helmholtz equation:

$$
\left(\nabla^{2}+k^{2}\right) f(\vec{r})=0
$$

where $k=2 \pi / \lambda$ is the wave number. For homogeneous media, the wavelength and corresponding wave number is constant. For propagation in inhomogeneous medium, the wave number can be expressed as a function of the position $k(\vec{r})$.

Consider the wave propagation from aperture plane $z=0$ to plane $z=d$ as shown in Figure 3.1. By applying the integral theorem of Helmholtz and Kirchhoff, the wave diffraction by an aperture $\mathcal{A}$ in an infinite opaque screen 
yields the Huygens-Fresnel diffraction [31]

$$
g(\vec{v})=\int_{\mathcal{A}} h(\vec{v}, \vec{u}) f(\vec{u}) \mathrm{d} \vec{u}
$$

where $\vec{u}=(x, y)$ and $\vec{v}=(x, y)$ are position vectors in the transverse planes $z=0$ and $z=d, f(\vec{u})$ is the optical field at the aperture $\mathcal{A}$ and $h(\vec{v}, \vec{u})$ is the system impulse response [31]

$$
h(\vec{v}, \vec{u})=\frac{1}{j \lambda} \frac{\exp (j k r)}{r} \cos \theta
$$

where $r=\sqrt{\|\vec{u}+\vec{v}\|^{2}+d^{2}}$ is the distance from point $(\vec{u}, 0)$ to point $(\vec{v}, d)$.

The scalar diffraction can also be analyzed in the Fourier domain. Various spatial Fourier components of the complex field can be identified as plane waves traveling in different directions [31]. It has been shown that wave propagation is a linear shift invariant system when the direction cosines $(\alpha, \beta)$ satisfy

$$
\alpha^{2}+\beta^{2}<1,
$$

where

$$
\alpha=\lambda \nu_{x}, \quad \beta=\lambda \nu_{y} .
$$

Then the propagation of angular spectrum can be described as a linear spatial filter with the transfer function

$$
H\left(\nu_{x}, \nu_{y}\right)=\left\{\begin{array}{ll}
\exp \left[j k d \sqrt{1-\left(\lambda \nu_{x}\right)^{2}-\left(\lambda \nu_{y}\right)^{2}}\right] & \nu_{x}^{2}+\nu_{y}^{2}<\frac{1}{\lambda^{2}} \\
0 & \text { otherwise }
\end{array},\right.
$$

where $\nu_{x}$ and $\nu_{y}$ are the spatial frequencies. 
When the propagation distance $d$ is much greater than the maximum linear dimension of both the aperture $\mathcal{A}$ and the observe region $\mathcal{R}$, and only a finite region around the $z$ axis is of interest, the following approximations can be used

$$
\begin{aligned}
\cos \theta & \approx 1 \\
\frac{1}{r} & \approx \frac{1}{d} \\
\exp (j k r) & \approx \exp \left[j k\left(d+\|\vec{v}-\vec{u}\|^{2} /(2 d)\right)\right] .
\end{aligned}
$$

Then we get the Green's function of Fresnel diffraction as

$$
h(\vec{v}, \vec{u}) \simeq \frac{e^{j k d}}{j \lambda d} e^{j \frac{\pi}{\lambda d}\|\vec{v}-\vec{u}\|^{2}}
$$

\subsection{Optical Beams}

\subsubsection{Paraxial waves and Gaussian beam}

We are interested primarily in the paraxial waves because they form beamlike optical waves. The complex amplitude of a paraxial wave can be expressed as

$$
f(\vec{u}, z)=a(\vec{u}, z) e^{j k z}
$$

where $a(\vec{u}, z)$ is a slowly varying function and $\vec{u}=(x, y)$ is the position vector in plane of constant $z$. Substituting the paraxial wave function into the Helmholtz equation, and making the assumption that

$$
\frac{\partial a}{\partial z} \ll k a, \quad \frac{\partial^{2} a}{\partial^{2} z} \ll k^{2} a
$$


we find that paraxial waves satisfy the following paraxial Helmhotz equation

$$
\nabla_{T}^{2} a-j 2 k \frac{\partial a}{\partial z}=0
$$

where $\nabla_{T}^{2}=\partial^{2} / \partial x^{2}+\partial^{2} / \partial y^{2}$ is the transverse Laplacian operator.

There are many solutions of the paraxial Helmhotz equation. A common example is a uniform spherical wave diverging from the origin with complex amplitude

$$
a(\vec{u}, z)=\frac{1}{z} \exp \left(-j k \frac{\|\vec{u}\|^{2}}{2 z}\right)
$$

where $\|\vec{u}\|^{2}=x^{2}+y^{2}$. Replacing the radius of curvature $z$ by the complex radius $q(z)=z+j z_{0}$ in the above equation, we get the common Gaussian beam:

$$
a(\vec{u}, z)=\frac{1}{q(z)} \exp \left(-j k \frac{\|\vec{u}\|^{2}}{2 q(z)}\right)
$$

where $z_{0}$ is the Rayleigh range. By separating the real and imaginary part of $1 / q(z)$ as

$$
\frac{1}{q(z)}=\frac{1}{R(z)}-j \frac{\lambda}{\pi w^{2}(z)}
$$

the Gaussian beam can be expressed as [60]:

$$
u(\vec{u}, z)=\frac{1}{j z_{0}} \frac{w_{0}}{w(z)} \exp \left(-\frac{\|\vec{u}\|^{2}}{w^{2}(z)}\right) \exp \left[-j k z-j k \frac{\|\vec{u}\|^{2}}{2 R(z)}+j \zeta(z)\right]
$$


where

$$
\begin{aligned}
w(z) & =w_{0}\left[1+\left(\frac{z}{z_{0}}\right)^{2}\right]^{1 / 2}, \\
R(z) & =z\left[1+\left(\frac{z_{0}}{z}\right)^{2}\right], \\
\zeta(z) & =\tan ^{-1} \frac{z}{z_{0}} \\
w_{0} & =\left(\frac{\lambda z_{0}}{\pi}\right)^{1 / 2} .
\end{aligned}
$$

The Gaussian beam is the lowest order solution in an infinite solution family of the free space paraxial wave equation. There are higher order solutions sets for the paraxial Helmhotz equation, such as the Hermite-Gaussian beams in rectangular coordinates and the Laguerre-Gaussian beam in cylindrical coordinates.

\subsubsection{Hermite-Gaussian beams}

The Hermite-Gaussian beams are the most widely used solution set of the paraxial wave equation in rectangular coordinates. The complex amplitude for Hermite-Gaussian beams can be expressed as [67]

$$
\begin{aligned}
f_{l, m}(x, y, z)= & a_{l, m} \frac{w_{0}}{w(z)} H_{l}\left(\sqrt{2} \frac{x}{w(z)}\right) H_{m}\left(\sqrt{2} \frac{y}{w(z)}\right) \\
& \times \exp \left[-\frac{r^{2}}{w^{2}(z)}-j k \frac{r^{2}}{2 R(z)}-j k z+j(l+m+1) \zeta(z)\right],
\end{aligned}
$$

where $r=\sqrt{x^{2}+y^{2}}, H_{m}$ is the Hermite polynomial of order $m$ :

$$
H_{m+1}(x)=2 x H_{m}(x)-2 m H_{m-1}(x),
$$


with

$$
H_{0}(x)=1, \quad \text { and } \quad H_{1}(x)=2 x \text {. }
$$

A plot of the transverse amplitude of the Hermit-Gaussian beams is shown in Figure 3.2, for $l=0,1,2,3$ and $m=0,1,2,3$.

\subsubsection{Laguerre-Gaussian beams}

The set of Laguerre-Gaussian beams is another complete solution of the paraxial wave equation expanded in cylindrical coordinates. The complex amplitude of Laguerre-Gaussian modes take the form [67]

$$
\begin{aligned}
f_{p, m}(r, \theta, z)= & \sqrt{\frac{2 p !}{\left(1+\delta_{0 m}\right) \pi(m+p) !}} \frac{\exp \left[j(2 p+m+1)\left(\zeta(z)-\zeta_{0}\right)\right]}{w(z)} \\
& \times\left(\frac{\sqrt{2} r}{w(z)}\right)^{m} L_{p}^{m}\left(\frac{2 r^{2}}{w^{2}(z)}\right) \exp \left[-j k \frac{r^{2}}{2 q(z)}+i m \theta\right],
\end{aligned}
$$

where

$$
L_{p}^{m}(x)=\sum_{k=0}^{p} \frac{(p+m) !}{(m+k) !(p-k) ! k !}(-x)^{k}
$$

is the generalized Laguerre polynomials and $1 / q(z)=\sqrt{2} / w(z)$. In the above solution, the integer $p \geq 0$ is the radial index and the integer $m$ is the azimuthal mode index. A plot of the transverse distribution of the LaguerreGaussian beams is shown in Figure 3.3, for $p=0,1,2,3$ and $m=0,1,2,3$. 

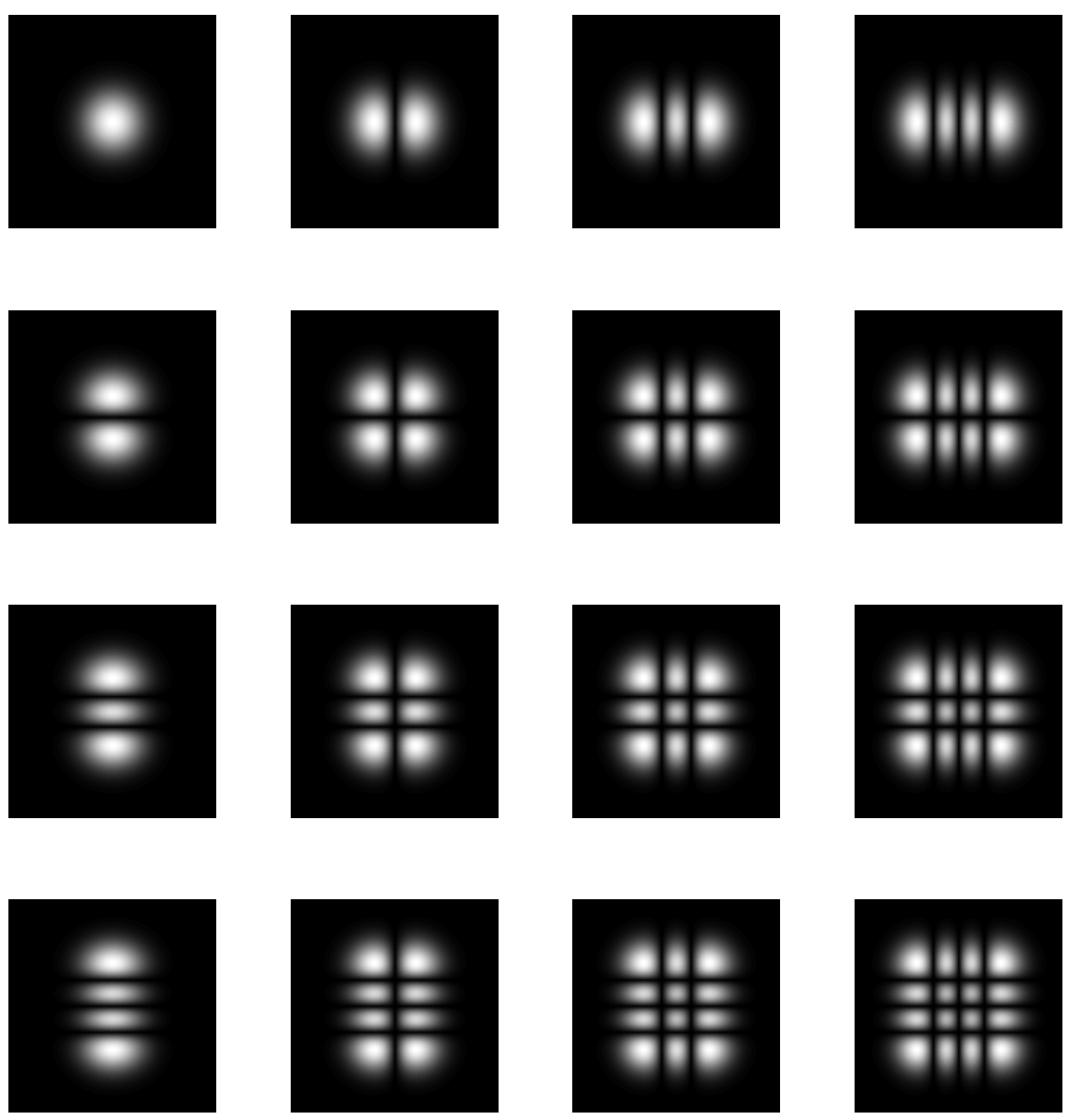

Figure 3.2: Hermite Gaussian beams $f_{l, m}(x, y)$ with $l=0,1,2,3$ for columns from left to right and $m=0,1,2,3$ for rows from top to bottom . 

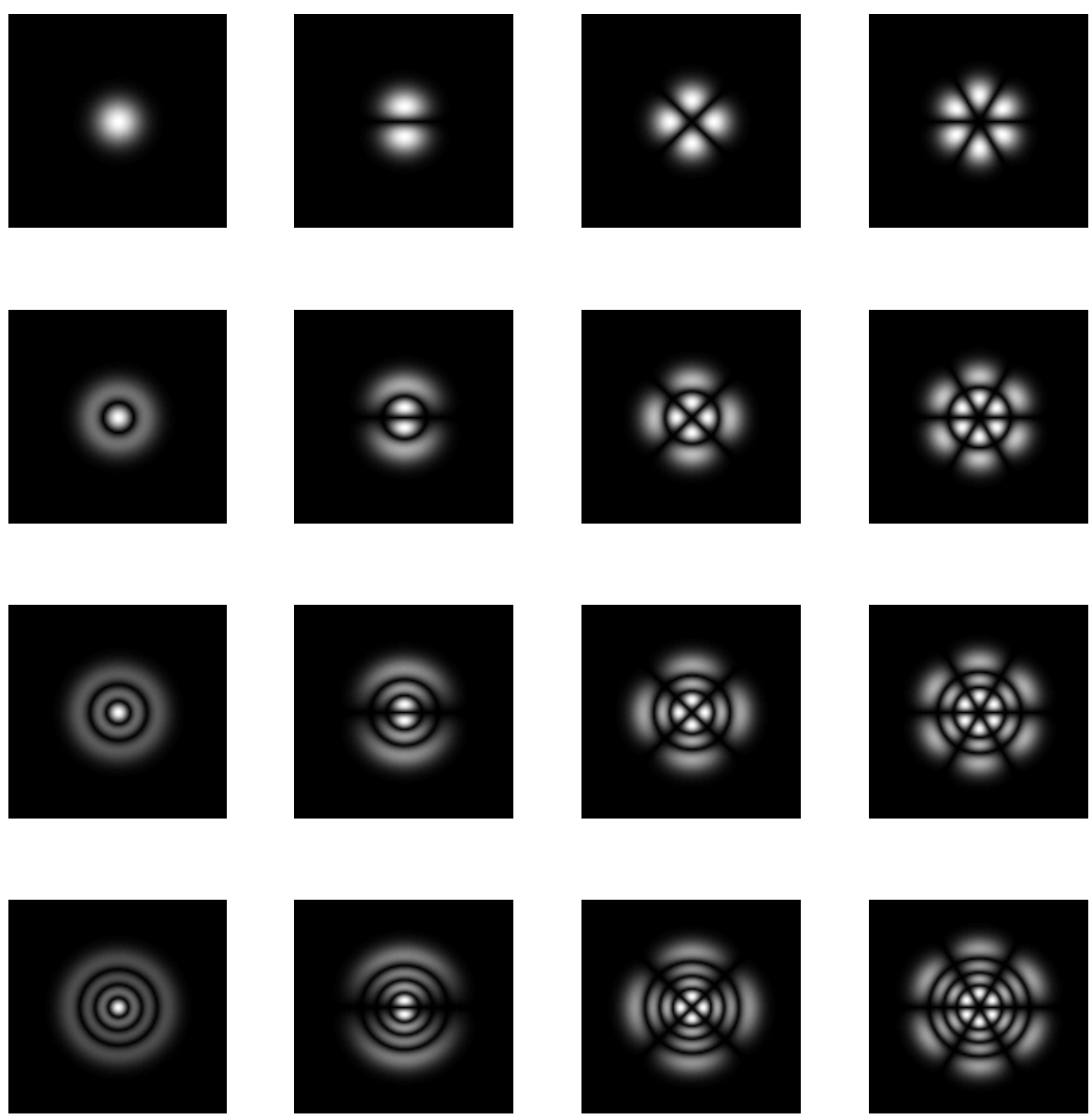

Figure 3.3: Laguerre Gaussian beams $f_{p, m}(r, \theta)$ with $p=0,1,2,3$ for rows from top to bottom and $m=0,1,2,3$ for columns from left to right. 


\subsection{Optical Coherence}

The optical coherence theory is used to describe the statistical fluctuations of an optical field by using coherence functions, and is very important in the study of optical beam propagation through turbulence. Basic concepts and methods of coherent optics are introduced in this section. We follow the notations in [50].

\subsubsection{Mutual coherence function}

Perhaps the most important concept in statistical optics is the mutual coherence, which is defined as the cross correlation function of two optical fields

$$
\Gamma\left(\vec{r}_{1}, \vec{r}_{2} ; t_{1}, t_{2}\right)=\left\langle f^{*}\left(\vec{r}_{1}, t_{1}\right) f\left(\vec{r}_{2}, t_{2}\right)\right\rangle
$$

where ${ }^{*}$ denotes the complex conjugate and $\langle\cdot\rangle$ denotes ensemble average. For stationary fields, the mutual coherence becomes

$$
\Gamma\left(\vec{r}_{1}, \vec{r}_{2} ; \tau\right)=\left\langle f^{*}\left(\vec{r}_{1}, t\right) f\left(\vec{r}_{2}, t+\tau\right)\right\rangle
$$

There are two types of coherence, temporal coherence characterized by $\Gamma(\vec{r}, \vec{r}, \tau)$ and spatial coherence characterized by $\Gamma\left(\vec{r}_{1}, \vec{r}_{2}, 0\right)$. The average field intensity is expressed in terms of the coherence as

$$
i(\vec{r}, t)=\Gamma(\vec{r}, \vec{r}, 0)=\left\langle f^{*}(\vec{r}, t) f(\vec{r}, t)\right\rangle
$$

From the relationship between correlation functions of complex analytic random processes, the cross correlation function $\Gamma\left(\vec{r}_{1}, \vec{r}_{2} ; \tau\right)$ of an analytic func- 
tion $f(\vec{r}, t)$ is also an analytic function and its real and imaginary part form a Hilbert transform pair [50]:

$$
\operatorname{Im} \Gamma\left(\vec{r}_{1}, \vec{r}_{2} ; \tau\right)=\frac{1}{\pi} P \int_{-\infty}^{\infty} \frac{\operatorname{Re} \Gamma\left(\vec{r}_{1}, \vec{r}_{2} ; \tau^{\prime}\right)}{\tau^{\prime}-\tau} \mathrm{d} \tau^{\prime}
$$

and

$$
\operatorname{Re} \Gamma\left(\vec{r}_{1}, \vec{r}_{2} ; \tau\right)=-\frac{1}{\pi} P \int_{-\infty}^{\infty} \frac{\operatorname{Im} \Gamma\left(\vec{r}_{1}, \vec{r}_{2} ; \tau^{\prime}\right)}{\tau^{\prime}-\tau} \mathrm{d} \tau^{\prime}
$$

where $P$ denotes the Cauchy principle value of the integrals at $\tau^{\prime}=\tau$.

A field's mutual intensity is defined as its correlation function at a time lag of zero:

$$
J\left(\vec{r}_{1}, \vec{r}_{2}\right)=\Gamma\left(\vec{r}_{1}, \vec{r}_{2}, 0\right)
$$

To measure a field's degree of coherence, the complex degree of coherence is defined as the normalized mutual coherence function

$$
\gamma\left(\vec{r}_{1}, \vec{r}_{2} ; \tau\right)=\frac{\Gamma\left(\vec{r}_{1}, \vec{r}_{2} ; \tau\right)}{\left[\Gamma\left(\vec{r}_{1}, \vec{r}_{1} ; 0\right)\right]^{1 / 2}\left[\Gamma\left(\vec{r}_{2}, \vec{r}_{2} ; 0\right)\right]^{1 / 2}}
$$

It can be seen that

$$
0 \leq\left|\gamma\left(\vec{r}_{1}, \vec{r}_{2} ; \tau\right)\right| \leq 1
$$

Mutual coherence can also be measured in the frequency domain. The cross spectral density (or cross power spectrum) is defined as the correlation of the spectral amplitudes at frequency $\nu$ :

$$
W\left(\vec{r}_{1}, \vec{r}_{2} ; \nu\right)=\left\langle F^{*}\left(\vec{r}_{1}, \nu\right) F\left(\vec{r}_{2}, \nu\right)\right\rangle
$$


where $F(\vec{r}, \nu)$ is the Fourier transform of field $f(\vec{r}, t)$ :

$$
f(\vec{r}, t)=\int_{0}^{\infty} F^{*}(\vec{r}, \nu) e^{-j 2 \pi \nu t} \mathrm{~d} \nu .
$$

Then the cross spectral density and the mutual coherence function form a Fourier transform pair

$$
\begin{aligned}
\Gamma\left(\vec{r}_{1}, \vec{r}_{2}, \tau\right) & =\int_{0}^{\infty} W\left(\vec{r}_{1}, \vec{r}_{2} ; \nu\right) e^{-j 2 \pi \nu \tau} \mathrm{d} \nu, \\
W\left(\vec{r}_{1}, \vec{r}_{2}, \nu\right) & =\int_{-\infty}^{\infty} \Gamma\left(\vec{r}_{1}, \vec{r}_{2} ; \tau\right) e^{j 2 \pi \nu \tau} \mathrm{d} \tau .
\end{aligned}
$$

The spectral density (or power spectrum) $S(\vec{r}, \nu)$ of light is a special case of the cross spectral density $W\left(\vec{r}_{1}, \vec{r}_{2}, \nu\right)$ at $\vec{r}_{1}=\vec{r}_{2}$

$$
S(\vec{r}, \nu)=W(\vec{r}, \vec{r}, \nu)
$$

The spectral degree of coherence is defined as the normalized cross spectral density function

$$
\mu\left(\vec{r}_{1}, \vec{r}_{2} ; \nu\right)=\frac{W\left(\vec{r}_{1}, \vec{r}_{2} ; \nu\right)}{\left[W\left(\vec{r}_{1}, \vec{r}_{1} ; \nu\right)\right]^{1 / 2}\left[W\left(\vec{r}_{2}, \vec{r}_{2} ; \nu\right)\right]^{1 / 2}}
$$

The degree of coherence can be measured by the value of $\left|\gamma\left(\vec{r}_{1}, \vec{r}_{2} ; \tau\right)\right|$. Fully coherent and fully incoherent are two special cases that occur when the value of $\left|\gamma\left(\vec{r}_{1}, \vec{r}_{2} ; \tau\right)\right|$ approaches its two extremes: 1 and 0 , respectively. Other states of coherence are called partially coherent. 


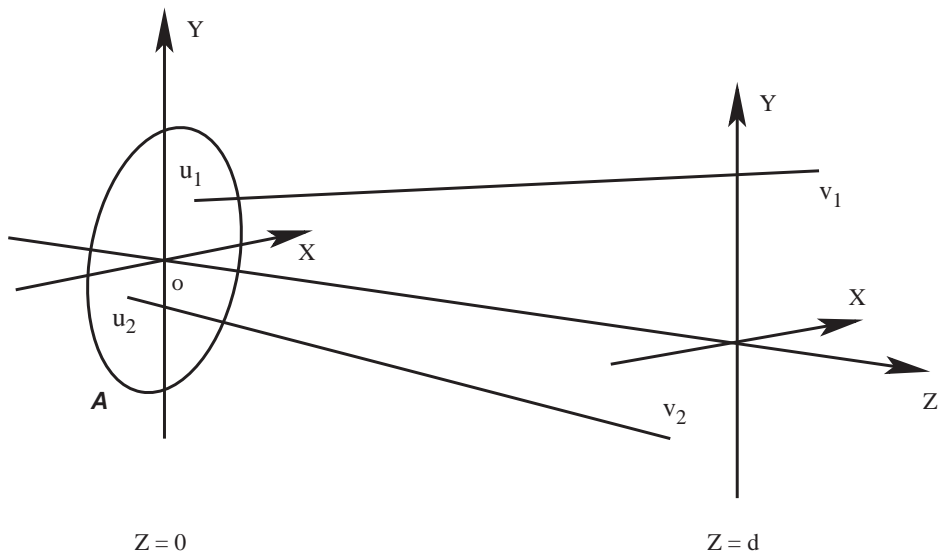

Figure 3.4: Propagation of optical coherence.

\subsubsection{Propagation of coherence}

To express the state changes of the optical coherence along propagation, the wave equations for mutual coherence propagation in free space were developed by Wolf [50]

$$
\begin{aligned}
& \nabla_{1}^{2} \Gamma\left(\vec{r}_{1}, \vec{r}_{2} ; \tau\right)=\frac{1}{c^{2}} \frac{\partial^{2}}{\partial \tau^{2}} \Gamma\left(\vec{r}_{1}, \vec{r}_{2} ; \tau\right) \\
& \nabla_{2}^{2} \Gamma\left(\vec{r}_{1}, \vec{r}_{2} ; \tau\right)=\frac{1}{c^{2}} \frac{\partial^{2}}{\partial \tau^{2}} \Gamma\left(\vec{r}_{1}, \vec{r}_{2} ; \tau\right),
\end{aligned}
$$

where $\nabla_{1}^{2}$ and $\nabla_{2}^{2}$ are the Laplacian operators taken with respect to $\vec{r}_{1}$ and $\vec{r}_{2}$. Taking the Fourier transform of the above equations yields the Helmholtz equations for monochromatic waves

$$
\begin{aligned}
& \left(\nabla_{1}^{2}+k^{2}\right) W\left(\vec{r}_{1}, \vec{r}_{2} ; \nu\right)=0, \\
& \left(\nabla_{2}^{2}+k^{2}\right) W\left(\vec{r}_{1}, \vec{r}_{2} ; \nu\right)=0 .
\end{aligned}
$$

Consider the propagation scheme as shown in Figure 3.4. For propagation 
from finite surfaces with small diffraction angle, the mutual coherence and the cross spectral density in the far field can be derived as

$$
\begin{aligned}
W\left(\vec{v}_{1}, \vec{v}_{2} ; \nu\right) & \approx \iint_{\mathcal{A}^{2}} W\left(\vec{u}_{1}, \vec{u}_{2} ; \nu\right) h^{*}\left(\vec{v}_{1}, \vec{u}_{1} ; \nu\right) h\left(\vec{v}_{2}, \vec{u}_{2} ; \nu\right) \mathrm{d} \vec{u}_{1} \mathrm{~d} \vec{u}_{2},(3 \\
\Gamma\left(\vec{v}_{1}, \vec{v}_{2} ; \tau\right) & \approx \iint_{\mathcal{A}^{2}} \Gamma\left(\vec{v}_{1}, \vec{v}_{2} ; \tau\right) h^{*}\left(\vec{v}_{1}, \vec{u}_{1}\right) h\left(\vec{v}_{2}, \vec{u}_{2}\right) \mathrm{d} \vec{u}_{1} \mathrm{~d} \vec{u}_{2}
\end{aligned}
$$

where $h(\vec{v}, \vec{u})$ is the impulse response function of wave propagation and $\tau=$ $\left(d_{1}-d_{2}\right) / c$. When $\tau=0$, Zernike's propagation law [50] for the mutual intensity is derived as

$$
J\left(\vec{v}_{1}, \vec{v}_{2}\right)=\iint_{\mathcal{A}^{2}} J\left(\vec{u}_{1}, \vec{u}_{2}\right) h^{*}\left(\vec{v}_{1}, \vec{u}_{1}\right) h\left(\vec{v}_{2}, \vec{u}_{2}\right) \mathrm{d} \vec{u}_{1} \mathrm{~d} \vec{u}_{2}
$$

For a planar and spatially incoherent source at $\mathcal{A}$, assuming that Fresnel approximation is valid, the mutual intensity in the far field can be derived as

$$
J\left(\vec{v}_{1}, \vec{v}_{2}\right)=\left(\frac{1}{\lambda d}\right)^{2} e^{-j \frac{\pi}{\lambda d}\left(\left|\vec{v}_{2}\right|^{2}-\left|\vec{v}_{1}\right|^{2}\right)} \int_{\mathcal{A}} i(\vec{u}) e^{-j k\left(\vec{v}_{2}-\vec{v}_{1}\right) \cdot \vec{u}} \mathrm{~d} \vec{u}
$$

This is the so called van Citter-Zernike theorem [30].

\subsubsection{Coherent mode representation}

We know that for a stationary optical field $f(\vec{r}, t)$ in some finite closed domain $D$ in free space, its mutual coherence function $\Gamma\left(\vec{r}_{1}, \vec{r}_{2}, \tau\right)$ and the cross spectral density function $W\left(\vec{r}_{1}, \vec{r}_{2}, \nu\right)$ form Fourier transform pairs

$$
W\left(\vec{r}_{1}, \vec{r}_{2}, \nu\right)=\int_{-\infty}^{\infty} \Gamma\left(\vec{r}_{1}, \vec{r}_{2}, \tau\right) e^{2 \pi \nu \tau} \mathrm{d} \tau
$$


It has been shown that the cross-spectral density function $W\left(\vec{r}_{1}, \vec{r}_{2}, \nu\right)$ is a Hilbert-Schmidt, Hermitian and non-negative definite kernel [50], which means that it has the Mercer expansion as [16]

$$
W\left(\vec{r}_{1}, \vec{r}_{2}, \nu\right)=\sum_{n} \alpha_{n}(\nu) \psi_{n}^{*}\left(\vec{r}_{1}, \nu\right) \psi_{n}\left(\vec{r}_{2}, \nu\right)
$$

where the functions $\psi_{n}(\vec{r}, \nu)$ are the eigenfunctions and $\alpha_{n}(\nu)$ are the eigenvalues of the integral equation

$$
\int_{D} W\left(\vec{r}_{1}, \vec{r}_{2}, \nu\right) \psi_{n}\left(\vec{r}_{1}, \nu\right) \mathrm{d} \vec{r}_{1}=\alpha_{n}(\nu) \psi_{n}\left(\vec{r}_{2}, \nu\right)
$$

Because the kernel $W\left(\vec{r}_{1}, \vec{r}_{2}, \nu\right)$ is Hermitian and non-negative definite, all its eigenvalues $\alpha_{n}(\nu)$ are real and non-negative and the eigenfunctions $\psi_{n}(\vec{r}, \nu)$ form an orthonormal set.

\subsection{Statistical Model of Atmospheric Turbu- lence}

Because of its nonlinear, non-closure (more variables than equations) and random nature [7], the turbulence phenomenon is very difficult to describe mathematically and can best be investigated using statistical methods. In this section, we introduce the most commonly used statistical models for atmospheric turbulence. 


\subsubsection{Kolmogorov turbulence model}

Kolmogorov investigated the mechanical structure of turbulence, and proposed a widely accepted model on the basis of a set of hypotheses [44, 45]. In Kolmogorov's model, the energy dissipation in fluid medium is from source to large structures, and then to small structures. For example, in the atmospheric turbulence, energy moves from the sun heating, through large scale disturbances with outer scale $L_{0}$, which then break down into smaller and smaller structures. These disturbances with different scale sizes are modeled as "eddies" with statistically varying index of refraction. The continuing kinetic energy transformation (energy cascade) stops and the kinetic energy dissipates into heat when the scale size of eddies approach the inner scale $l_{0}$, where the Reynolds number drops below its critical value and the turbulence dies away.

For eddies with scale size $l$ within the inertial subrange (ie., $l_{0} \leq l \leq L_{0}$ ), Kolmogorov showed that the structure function of turbulence conforms to a universal "two-third law":

$$
D_{f}(\tau)=C_{f}^{2} \tau^{2 / 3},
$$

where the structure function $D_{f}(\tau)$ of a random process $f(t)$ was introduced by Kolmogorov as

$$
D_{f}(\tau)=\left\langle[f(t+\tau)-f(t)]^{2}\right\rangle .
$$

The structure function $D_{f}(\tau)$ can filter the slow fluctuations of the random 
process. The fluctuations about the varying mean $f(t+\tau)-f(t)$ can be modeled as a stationary process (when $\tau$ is not too large) even though $f(t)$ is not. This is referred to as possessing stationary increments in the time domain, or locally homogeneous in the spatial domain. For locally homogenous random processes, the spectrum is related to the structure function by the generalized Wiener-Khinchine theorem

$$
D_{f}(\vec{r})=2 \int_{-\infty}^{\infty} \Phi_{f}(\vec{\kappa})[1-\cos (\vec{\kappa} \cdot \vec{r})] \mathrm{d} \vec{\kappa}
$$

where $\vec{\kappa}$ is the vector wave number. For isotropic processes we have

$$
D_{f}(r)=8 \pi \int_{0}^{\infty} \Phi_{f}(\kappa)\left[1-\frac{\sin (\kappa r)}{\kappa r}\right] \kappa^{2} \mathrm{~d} \kappa
$$

and

$$
\Phi_{f}(\kappa)=\frac{1}{4 \pi^{2} \kappa^{2}} \int_{0}^{\infty} \frac{\sin (\kappa r)}{\kappa r} \frac{\mathrm{d}}{\mathrm{d} r}\left[r^{2} \frac{\mathrm{d} D_{f}(r)}{\mathrm{d} r}\right] \mathrm{d} r .
$$

\subsubsection{Power spectrum of refractive index fluctuations}

As we have discussed, refractive index fluctuations induced by solar heating are the main reason for atmospheric turbulence effects on optical beam propagation. The refractive index of the atmosphere can be expressed as [53]

$$
n=1+a_{d}(\lambda) \frac{P_{d}}{T}+a_{w}(\lambda) \frac{P_{w}}{T}
$$

where $a_{d}(\lambda)$ is a wavelength dependent function and $P_{d}$ is artial pressure (in mbar) of the dry air. $a_{w}(\lambda)$ and $P_{w}$ are those for water vapor, and $T$ is the absolute temperature in Kelvin. According to Kolmogorov's theory, the 
structure function of refractive index can be expressed as

$$
D_{n}(r)=C_{n}^{2} r^{2 / 3}
$$

where $C_{n}^{2}$ is the structure constant of the index of refraction. This is the critical parameter for describing optical turbulence. Substituting this model into the Winer-Khinchine theorems yields the spectrum within the inertial range as

$$
\Phi_{n}(\kappa)=\frac{5}{18 \pi} C_{n}^{2} \kappa \int_{l_{0}}^{L_{0}} r^{-1 / 3} \sin (\kappa) r \mathrm{~d} r .
$$

In the case that $2 \pi / L_{0} \ll \kappa \ll 2 \pi / l_{0}$, with the integration limits approximated from 0 to $\infty$, we obtain the Kolmogorov spectrum of the refractive index fluctuations

$$
\Phi_{n}(\kappa)=0.033 C_{n}^{2} \kappa^{-11 / 3}
$$

To resolve the deficiency with large wave numbers in the above form, Tatarski [73] modified it as

$$
\Phi_{n}(\kappa)=0.033 C_{n}^{2} \kappa^{-11 / 3} \exp \left(-\frac{\kappa^{2}}{\kappa_{m}^{2}}\right)
$$

where $\kappa_{m}=5.92 / l_{0}$ is an inner scale related constant. However the singularity at $\kappa=0$ is still there. This problem is solved in the modified von Karman spectrum with improved range of usefulness

$$
\Phi_{n}(\kappa)=\frac{0.033 C_{n}^{2}}{\left(\kappa^{2}+\kappa_{0}^{2}\right)^{11 / 6}} e^{-\kappa^{2} / \kappa_{m}^{2}},
$$


where $\kappa_{0}=2 \pi / L_{0}$ and $\kappa_{m}=5.92 / l_{0}$. It should be noted that these modifications are for computational, but not physical, reasons.

\subsection{Wave Propagation through Turbulence}

Turbulence causes spatial and temporal random fluctuations in the index of refraction which distorts the Green's function for a propagation field. To model the propagation of optical waves through turbulence, researchers developed many methods to provide appropriate statistical descriptions of the propagated field. In this section, we will introduce the models that are used in our investigation of the optimal beam through random media.

\subsubsection{Inhomogeneous wave equation}

As mentioned in Section 3.1, scalar field propagation in homogeneous media is governed by the Helmholtz equation

$$
\left(\nabla^{2}+k^{2}\right) f(\vec{r})=0
$$

For propagation in inhomogeneous media, the wave number $k$ is a function of the position $\vec{r}$, and the above equation can be modified to form the inhomogeneous wave equation as

$$
\left(\nabla^{2}+k^{2}(\vec{r})\right) f(\vec{r})=0
$$


The wave number $k(\vec{r})$ can be expressed as a scalar function of the refractive index $n(\vec{r})$

$$
k(\vec{r})=k_{0} n(\vec{r})
$$

where $k_{0}$ represents the average wave number of the medium and $n(\vec{r})$ is the refractive index of the medium given by

$$
n(\vec{r})=\sqrt{\frac{\mu(\vec{r}) \epsilon(\vec{r})}{\mu_{0} \epsilon_{0}}},
$$

where $\mu$ and $\epsilon$ are the magnetic permeability and dielectric constant of the medium, and $\mu_{0}$ and $\epsilon_{0}$ are corresponding average values. The refractive index $n(\vec{r})$ is a random variable and can be written in the average and fluctuation terms as

$$
n(\vec{r})=\langle n\rangle+n_{s}(\vec{r})
$$

In general cases such as propagation in the atmosphere, we can assume that $\langle n\rangle=1$. Then the wave equation becomes

$$
\nabla^{2} f(\vec{r})+k_{0}^{2}\left[1+\delta_{n}(\vec{r})\right] f(\vec{r})=0
$$

where

$$
\delta_{n}(\vec{r})=2 n_{s}(\vec{r})+n_{s}^{2}(\vec{r}) .
$$

It is difficult to solve this inhomogeneous wave equation analytically. Several approximations have been developed for certain turbulence cases and solutions for these approximations can be derived. 


\subsubsection{Born approximation}

In the Born approximation, the optical field $f(\vec{v})$ is expressed as a sum of the field in free space, $f_{0}(\vec{v})$, and the perturbation term, $f_{s}(\vec{v})$,

$$
f(\vec{v})=f_{0}(\vec{v})+f_{s}(\vec{v}) .
$$

Substituting the above representation into the wave equation (3.71), we have

$$
\left(\nabla^{2}+k_{0}^{2}\right) f_{s}(\vec{v})=-k_{0}^{2} \delta n(\vec{v}) f(\vec{v})
$$

This equation can not be solved directly, but the solution can be written in terms of the Green's function as

$$
f_{s}(\vec{v})=\int k_{0}^{2} \delta_{n}(\vec{u}) G(\vec{v}-\vec{u}) f(\vec{u}) \mathrm{d} \vec{u}
$$

where $G(\vec{v}-\vec{u})$ is the Green's function of the wave equation (3.74), a solution of the differential equation

$$
\left(\nabla^{2}+k_{0}^{2}\right) G(\vec{v}, \vec{u})=-\delta(\vec{v}-\vec{u}) .
$$

It can be shown that the Green's function is only a function of the difference $(\vec{v}-\vec{u})$. When the perturbation $f_{s}(\vec{v})$ is small compared with $f_{0}(\vec{v})$, the integral (3.75) can be approximated as

$$
f_{s}(\vec{v}) \simeq f_{B}(\vec{v})=\int k_{0}^{2} \delta_{n}(\vec{u}) G(\vec{v}-\vec{u}) f_{0}(\vec{u}) \mathrm{d} \vec{u} .
$$


An iterative method can then be taken to approach the final solution and the $i$ th order Born field can be derived as

$$
f_{B}^{(i+1)}(\vec{v})=\int k_{0}^{2} \delta_{n}(\vec{u}) G(\vec{v}-\vec{u})\left[f_{B}^{(i)}(\vec{u})+f_{0}(\vec{u})\right] \mathrm{d} \vec{u}
$$

In weak turbulence case, the first order Born approximation is valid and is widely taken because of its simplicity in calculation.

\subsubsection{Rytov approximation}

The Rytov method states that the optical fields in inhomogeneous media can be represented in the complex exponential form as

$$
f(\vec{r})=e^{\psi(\vec{r})},
$$

Substituting this into the inhomogeneous wave equation (3.71), we obtain a nonlinear first order differential equation for $\nabla \psi$, which is called the Riccati equation:

$$
\nabla^{2} \psi+\nabla \psi \cdot \nabla \psi+k^{2}\left(1+\delta_{n}\right)=0
$$

The complex phase $\psi$ can be written as

$$
\psi=\psi_{0}+\psi_{s}
$$

where

$$
f_{0}(\vec{r})=e^{\psi_{0}(\vec{r})}
$$

is the solution of the wave equation in the absence of turbulence and $\psi_{s}$ accounts for the random fluctuations. The Riccati equation (3.5.3) can be 
rewritten as

$$
\nabla^{2} \psi_{s}+2 \nabla \psi_{0} \cdot \nabla \psi_{s}+\left(\nabla \psi_{s}\right)^{2}+k_{0}^{2} \delta_{n}=0
$$

The solution of the fluctuation part $\psi_{s}$ can be found to be [37]

$$
\psi_{s}(\vec{r})=\frac{1}{f_{0}(\vec{r})} \int G(\vec{r}-\vec{u})\left[\left(\nabla \psi_{s}\right)^{2}+k^{2} \delta_{n}(\vec{u})\right] f_{0}(\vec{u}) \mathrm{d} \vec{u} .
$$

As in the Born approximation, this equation can be solved by iteration, yielding a sequence of solutions. In weak turbulence cases, assuming that

$$
\left(\nabla \psi_{s}\right)^{2} \ll k^{2} \delta_{n}(\vec{u})
$$

the first Rytov solution is derived:

$$
\psi_{s}(\vec{r})=\frac{1}{f_{0}(\vec{r})} \int G(\vec{r}-\vec{u}) k^{2} \delta_{n}(\vec{u}) f_{0}(\vec{u}) \mathrm{d} \vec{u}
$$

It has been shown that the conditions for the Rytov approximation to be valid are less restrictive than that for Born approximation [41]. For small perturbations, the Rytov approximation provides nearly the same results as the Born approximation.

The wave fields can also be expressed in the form as

$$
f(\vec{r})=a(\vec{r}) e^{j \phi(\vec{r})}
$$

and

$$
f_{0}(\vec{r})=a_{0}(\vec{r}) e^{j \phi_{0}(\vec{r})}
$$


We then get

$$
\psi_{s}(\vec{r})=\chi+j \phi_{s}=\ln \left(\frac{a}{a_{0}}\right)+j\left(\phi-\phi_{0}\right),
$$

where $\chi$ denotes the log-amplitude fluctuations and $\phi_{s}$ is the phase fluctuations. It will be seen that the following statistical properties of $\chi$ and $\phi_{s}$ are important:

$$
\begin{aligned}
& B_{\chi}\left(\vec{r}_{1}, \vec{r}_{2}\right)=\left\langle\chi\left(\vec{r}_{1}\right) \chi\left(\vec{r}_{2}\right)\right\rangle \\
& B_{\phi}\left(\vec{r}_{1}, \vec{r}_{2}\right)=\left\langle\phi_{s}\left(\vec{r}_{1}\right) \phi_{s}\left(\vec{r}_{2}\right)\right\rangle,
\end{aligned}
$$

and

$$
B_{\chi \phi}\left(\vec{r}_{1}, \vec{r}_{2}\right)=\left\langle\chi\left(\vec{r}_{1}\right) \phi_{s}\left(\vec{r}_{2}\right)\right\rangle
$$

These properties can be obtained by using the autocorrelation function of the index of refraction of the turbulent media

$$
B_{n}\left(\vec{r}_{1}, \vec{r}_{2}\right)=\left\langle n\left(\vec{r}_{1}\right) n\left(\vec{r}_{2}\right)\right\rangle,
$$

or the corresponding power spectrum of the index of refraction fluctuations. Since it simplifies the calculations and also provides another point of view, the spectral method is commonly used in practice.

\subsubsection{Extended Huygens-Fresnel principle}

In strong turbulence and long path propagation cases, the Born and Rytov approximations cannot be used. The extended Huygens-Fresnel (EHF) principle proposed by Lutomirski and Yura [49] demonstrated that the solution 
of the inhomogeneous wave equation can be expressed in a linear system representation. In contrast with previous work, the properties of the random medium appear only in the mutual coherence function of a spherical wave.

Applying the extended Huygens-Fresnel principle, the received field at distance $d$ is expressed as

$$
g(\vec{v})=\int_{\mathcal{A}} h(\vec{v}, \vec{u}) f(\vec{u}) \mathrm{d} \vec{u}
$$

where $h(\vec{v}, \vec{u})$ is the spatial impulse response of the system. Reciprocity of the response [64] implies that $h(\vec{v}, \vec{u})$ is symmetric with respect to the source point and the observer point. Therefore, a point source at $\vec{u}$ will produce at $\vec{v}$ the same effect that a point source of equal intensity at $\vec{v}$ will produce at $\vec{u}$. The impulse response in random media takes the form

$$
h(\vec{v}, \vec{u})=\frac{1}{j \lambda d} \exp \left(j \frac{2 \pi}{\lambda d}\right) \exp \left[j \frac{\pi}{\lambda d}\|\vec{v}-\vec{u}\|^{2}+\psi(\vec{v}, \vec{u})\right]
$$

where

$$
\psi(\vec{v}, \vec{u})=\chi(\vec{v}, \vec{u})+j \phi(\vec{v}, \vec{u})
$$

is the random part of the complex phase of a spherical wave propagating in the random medium from point $(\vec{u}, 0)$ to point $(\vec{v}, d)$. Then the intensity at target position $\vec{v}$ can be derived as

$$
\begin{aligned}
i(\vec{v})= & |g(\vec{v})|^{2} \\
= & \left(\frac{1}{\lambda d}\right)^{2} \iint_{\mathcal{A}^{2}} \exp \left[j \frac{\pi}{\lambda d}\left(\left\|\vec{v}-\vec{u}_{2}\right\|^{2}-\left\|\vec{v}-\vec{u}_{1}\right\|^{2}\right)\right] \\
& \times \exp \left[\psi^{*}\left(\vec{v}, \vec{u}_{1}\right)+\psi\left(\vec{v}, \vec{u}_{2}\right)\right] f^{*}\left(\vec{u}_{1}\right) f\left(\vec{u}_{2}\right) \mathrm{d} \vec{u}_{1} \mathrm{~d} \vec{u}_{2} .
\end{aligned}
$$


It can be seen that the ensemble average $\left\langle\exp \left[\psi^{*}\left(\vec{v}, \vec{u}_{1}\right)+\psi\left(\vec{v}, \vec{u}_{2}\right)\right]\right\rangle$ is needed to compute the mean irradiance $\langle i(\vec{v})\rangle$ from a finite diffracting aperture. Similarly, higher moments of $i(\vec{v})$ can be computed from a knowledge of terms of the form $\left\langle\exp \left[\psi^{*}\left(\vec{v}_{1}, \vec{u}_{1}\right)+\psi\left(\vec{v}_{1}, \vec{u}_{2}\right)+\psi^{*}\left(\vec{v}_{2}, \vec{u}_{3}\right)+\psi\left(\vec{v}_{2}, \vec{u}_{4}\right)\right]\right\rangle$, etc.

\subsubsection{Turbulence layer model}

In some applications, the random media can be modeled by thin turbulence layers between the transmitter and receiver. These turbulence layers can be modeled as thin screens, and the field that passes through each screen can be expressed as

$$
f(\vec{u})=f_{0}(\vec{u}) t_{s}(\vec{u})
$$

where $f_{0}(\vec{u})$ is the wave function before the phase screen and $t_{s}(\vec{u})$ is the screen transmittance. In general, $t_{s}(\vec{u})$ includes random changes in both phase and amplitude. Sometimes only phase perturbations are considered. Then we have the random phase screen model as

$$
f(\vec{u})=f_{0}(\vec{u}) e^{j \phi(\vec{u})}
$$

where $\phi(\vec{u})$ is the random phase change. Because of its mathematical simplification, the random phase screen model is widely used in areas such as wave propagation through the ionosphere, scattering from rough surfaces, and optical communications between the Earth and satellites.

The turbulence layer model is a geometrical optics model for wave propagation. It is assumed that there is no refraction inside the very thin tur- 


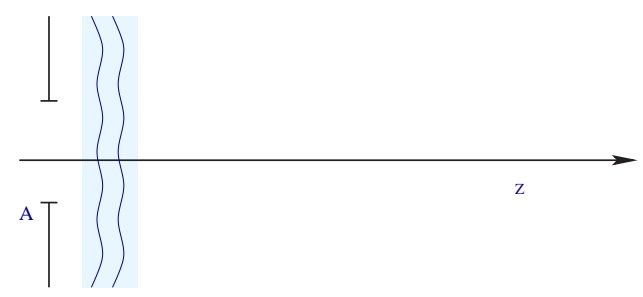

(a)

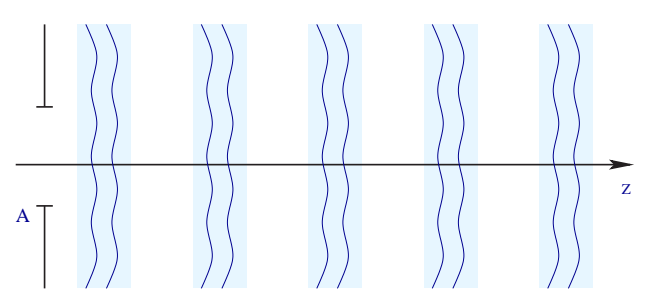

(b)

Figure 3.5: Turbulence layer propagation model: (a) Pupil plane phase screen model. (b) Multiple phase screen model.

bulence layer. The turbulence effect on wave propagation can be simulated by the pupil plane phase screen model or the multiple phase screens model. In the pupil plane phase screen model, the turbulence of the whole propagation path is modeled as a random phase screen at the transmitter pupil plane, as shown in Figure 3.5 (a). The pupil plane phase screen model is a simplified propagation model that is only appropriate for certain cases. For more complicated situations, multiple phase screens are used to simulate the turbulence effect as shown in Figure 3.5 (b).

\subsection{Performance Metrics}

To overcome the random media effects such as beam spread and intensity fluctuations, compensation schemes are investigated by modifying the transmitted fields. Various optimization criteria have been defined to evaluate the optical beam performance. In this study, two optimization criteria are introduced to measure the beam spread and scintillation effect. 


\subsubsection{Maximization of the integrated intensity}

For this criterion, the goal is to concentrate more power or energy at the receiver region through the propagation media. We define the integrated intensity at the receiver as the performance metric

$$
\mathcal{I}=\int w(\vec{v})|g(\vec{v})|^{2} \mathrm{~d} \vec{v}
$$

where $w(\vec{v})$ is the weighting function at the receiver region, and $g(\vec{v})$ is the received field at the target diffracted from the transmitted source field $f(\vec{u})$ at pupil aperture $\mathcal{A}$

$$
g(\vec{v})=\int_{\mathcal{A}} h(\vec{v}, \vec{u}) f(\vec{u}) \mathrm{d} \vec{u} .
$$

Accordingly, the optimal beam is defined to be the input field that maximizes this integrated intensity for a particular weighting function $w(\vec{v})$ :

$$
\hat{f}(\vec{u})=\arg \max _{\|f\|^{2}=\mathcal{I}_{0}} \mathcal{I}
$$

where $\|f\|^{2}=\mathcal{I}_{0}$ is the energy constraint for the source field:

$$
\|f\|^{2}=\int_{\mathcal{A}}|f(\vec{u})|^{2} \mathrm{~d} \vec{u} .
$$

\subsubsection{Minimization of the scintillation index}

To reduce the random fluctuations of the received fields, the "scintillation index" has been widely used in laser beam compensation [2]. The scintillation index of the received field has been defined as the normalized variance of 
intensity fluctuations:

$$
S_{\mathcal{I}}=\frac{\operatorname{var}[\mathcal{I}]}{(\mathrm{E}[\mathcal{I}])^{2}}=\frac{\mathrm{E}\left[\mathcal{I}^{2}\right]}{(\mathrm{E}[\mathcal{I}])^{2}}-1,
$$

where $\mathcal{I}$ is the integrated intensity at the receiver as defined in equation (3.100). Accordingly, the optimal beam has been defined as the transmitted optical field to minimize this scintillation index:

$$
\hat{f}(\vec{u})=\arg \min _{\|f\|^{2}=\mathcal{I}_{0}} S_{\mathcal{I}}
$$

There are several other metrics for laser beam performance measurement in random medium, such as beam width and on axis Strehl ratio. In this study, we will use the two criteria we introduced and discuss the optimal beams that corespond to them respectively. 


\section{Chapter 4}

\section{Optimal Beam to Maximize the Integrated Intensity}

In many optical applications, the goal is to concentrate the greatest percentage of the transmitted energy or power on the receiver. For example in laser weapon systems high power laser beams are used to disable or destroy military targets at far distances. In free space laser communication systems using direct detection, the detection process can be considered as a simple energy collection process in the receiver photodetector. In situations with random propagation media, system performance is degraded by the turbulence effect. In this chapter, we will discuss beams that are used to maximize the integrated energy or power through turbulence.

As shown in equation (3.102), the optimal beam is defined as the transmitted field $f(\vec{u})$ that maximizes the integrated weighted intensity $\mathcal{I}$ in the receiver region for a particular weighting function $w(\vec{v})$,

$$
\hat{f}(\vec{u})=\arg \max _{\|f\|^{2}=\mathcal{I}_{0}} \mathcal{I}
$$




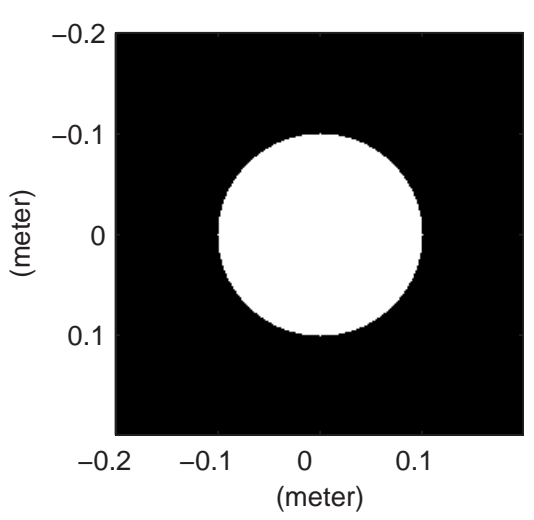

(a)

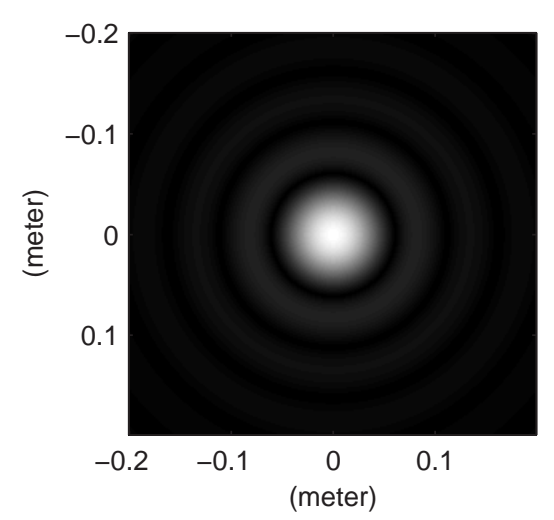

(b)

Figure 4.1: Free space diffraction of focused field from circular aperture: $\lambda=10^{-6}, d=10^{4}, D=0.2$, all units in meters. (a) Transmitting aperture $a(\vec{u})$. (b) Normalized amplitude of the received field $g(\vec{v})$.

where the weighted integrated intensity $\mathcal{I}$ has been defined in equation (3.100) as

$$
\mathcal{I}=\int w(\vec{v})|g(\vec{v})|^{2} \mathrm{~d} \vec{v}
$$

When the Fresnel approximation is valid, the received field $g(\vec{v})$ can be derived as

$$
g(\vec{v})=\int_{\mathcal{A}} h(\vec{v}, \vec{u}) f(\vec{u}) \mathrm{d} \vec{u}
$$

where $h(\vec{v}, \vec{u})$ is the propagation impulse function

$$
h(\vec{v}, \vec{u})=\frac{1}{j \lambda d} \exp \left(j \frac{2 \pi}{\lambda} d\right) \exp \left(j \frac{\pi}{\lambda d}\|\vec{v}-\vec{u}\|^{2}\right) .
$$

For example, consider the free space diffraction from a circular aperture 
$\mathcal{A}$ with aperture function

$$
a(\vec{u})=\left\{\begin{array}{ll}
1 & \|\vec{u}\| \leq D / 2 \\
0 & \|\vec{u}\|>D / 2
\end{array},\right.
$$

where $D$ is the diameter of the aperture. It is well known that when a focused beam is transmitted,

$$
f(\vec{u})=\exp \left(-j \frac{\pi}{\lambda d}\|\vec{u}\|^{2}\right)
$$

the intensity of the received field is the Airy pattern

$$
\begin{aligned}
|g(\vec{v})|^{2} & =\left(\frac{1}{\lambda d}\right)^{2}\left|\int a(\vec{u}) \exp \left(-j \frac{2 \pi}{\lambda d} \vec{v} \cdot \vec{u}\right) \mathrm{d} \vec{u}\right|^{2} \\
& =\left(\frac{1}{\lambda d}\right)^{2}\left|A\left(\frac{\vec{v}}{\lambda d}\right)\right|^{2}
\end{aligned}
$$

where $A(\vec{\nu})$ is the Fourier transform of the aperture function $a(\vec{u})$

$$
A(\vec{\nu})=\left(\frac{D}{2}\right)^{2} \frac{2 J_{1}(\pi D|\vec{\nu}|)}{D|\vec{\nu}|} .
$$

Illustrations of the transmitting aperture and the received field are shown in Figure 4.1.

To measure the integrated intensity inside a circular region $\mathcal{R}$ in the receiver, we define the weighting function as

$$
w(\vec{v})=\left\{\begin{array}{ll}
1 & \|\vec{v}\| \leq r \\
0 & \|\vec{v}\|>r
\end{array},\right.
$$

where $r$ is the radius of $\mathcal{R}$. Then the integrated intensity inside the receiver 


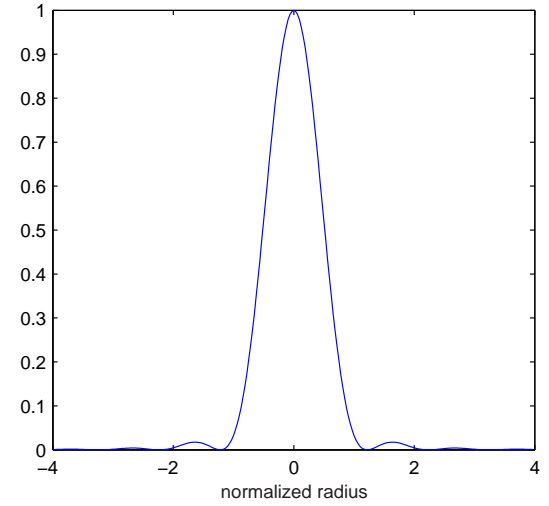

(a)

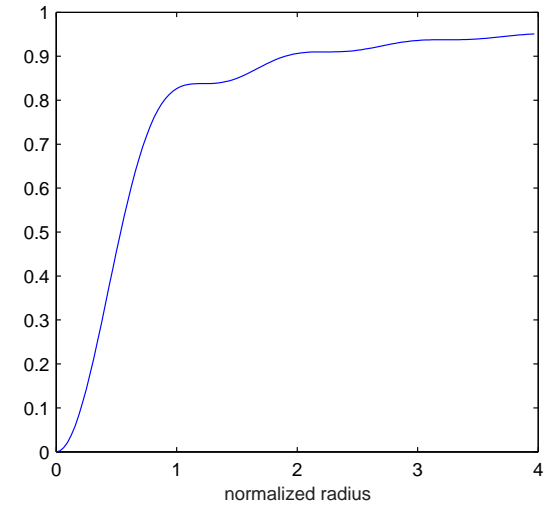

(b)

Figure 4.2: Airy pattern: (a)Normalized Airy pattern slice, (b)Fractional integrated intensity $\mathcal{I} / \mathcal{I}_{0}$ vs. normalized target plane radius $(r D) /(\lambda d)$

region $\mathcal{R}$ can be expressed as

$$
\begin{aligned}
\mathcal{I} & =\int w(\vec{v}) i(\vec{v}) \mathrm{d} \vec{v} \\
& =\int_{0}^{2 \pi} \int_{0}^{r} \frac{D^{2} J_{1}^{2}[\pi(D / \lambda d)|\rho|]}{4|\rho|^{2}} \rho \mathrm{d} \rho \mathrm{d} \theta .
\end{aligned}
$$

The normalized integrated intensity can be derived as [50]

$$
\frac{\mathcal{I}}{\mathcal{I}_{0}}=1-J_{0}^{2}\left(\pi \frac{D r}{\lambda d}\right)-J_{1}^{2}\left(\pi \frac{D r}{\lambda d}\right)
$$

where $J_{0}$ and $J_{1}$ are the first kind of Bessel functions of order 0 and 1, and $\mathcal{I}_{0}$ is the total transmitted intensity

$$
\mathcal{I}_{0}=\int a(\vec{u})|f(\vec{u})|^{2} \mathrm{~d} \vec{u}=\frac{\pi D^{2}}{4}
$$

A plot of the Airy pattern center slice and the integrated intensity is shown in Figure 4.2 


\subsection{Optimal Beam in Free Space}

The maximization of the fractional integrated intensity at the receiver is a radiation transportation problem that has been widely studied as a case of an "apodization" problem $[71,48]$. Apodization theory is concerned with the determination of the distribution of light over the exit pupil of an optical system required in order to achieve a desired distribution of illuminance over a given plane in the image field [68]. Most often the goal is suppression of the side lobes of the diffraction pattern. It is sometimes also called "tapering", a mathematical technique used to reduce the Gibbs phenomenon "ringing" that is produced in a spectrum obtained from a truncated interferogram [81]. A comprehensive review of the apodization problem has been given in reference [38].

The integrated intensity at the receiver can be expressed as

$$
\begin{aligned}
\mathcal{I} & =\int w(\vec{v})|g(\vec{v})|^{2} \mathrm{~d} \vec{v} \\
& =\int w(\vec{v})\left|\int_{\mathcal{A}} h(\vec{v}, \vec{u}) f(\vec{u}) \mathrm{d} \vec{u}\right|^{2} \mathrm{~d} \vec{v} \\
& =\iint f^{*}\left(\vec{u}_{1}\right) \mathcal{H}\left(\vec{u}_{1}, \vec{u}_{2}\right) f\left(\vec{u}_{2}\right) \mathrm{d} \vec{u}_{1} \mathrm{~d} \vec{u}_{2},
\end{aligned}
$$

where

$$
\mathcal{H}\left(\vec{u}_{1}, \vec{u}_{2}\right)=a^{*}\left(\vec{u}_{1}\right) \int w(\vec{v}) h^{*}\left(\vec{v}, \vec{u}_{1}\right) h\left(\vec{v}, \vec{u}_{2}\right) \mathrm{d} \vec{v} a\left(\vec{u}_{2}\right)
$$

This optimal beam has the same mathematical formalization as the apodization problem discussed in [68]. Two apodization problems in free space are 
discussed and complete solutions are given. Slepian [68] shows that the solution is the largest eigenvalue's eigenfunction for the integral equation

$$
\lambda \xi\left(\vec{u}_{1}\right)=\int \mathcal{H}\left(\vec{u}_{1}, \vec{u}_{2}\right) \xi\left(\vec{u}_{2}\right) \mathrm{d} \vec{u}_{2}
$$

In free space propagation with the Fresnel approximation, the kernel $\mathcal{H}_{0}\left(\vec{u}_{1}, \vec{u}_{2}\right)$ takes the form

$$
\begin{aligned}
\mathcal{H}_{0}\left(\vec{u}_{1}, \vec{u}_{2}\right) & =a^{*}\left(\vec{u}_{1}\right) \int h^{*}\left(\vec{v}, \vec{u}_{1}\right) h\left(\vec{v}, \vec{u}_{2}\right) w(\vec{v}) \mathrm{d} \vec{v} a\left(\vec{u}_{2}\right) \\
& =\frac{e^{-j \frac{\pi}{\lambda d}\left(\left\|\vec{u}_{1}\right\|^{2}-\left\|\vec{u}_{2}\right\|^{2}\right)}}{(\lambda d)^{2}} a^{*}\left(\vec{u}_{1}\right) a\left(\vec{u}_{2}\right) \int w(\vec{v}) e^{-j \frac{2 \pi}{\lambda d}\left(\vec{u}_{2}-\vec{u}_{1}\right) \cdot \vec{v}} \mathrm{~d} \vec{v} \\
& =\left.\left(\frac{1}{\lambda d}\right)^{2} e^{-j \frac{\pi}{\lambda d}\left(\left\|\vec{u}_{1}\right\|^{2}-\left\|\vec{u}_{2}\right\|^{2}\right)} a^{*}\left(\vec{u}_{1}\right) a\left(\vec{u}_{2}\right) W(\vec{\nu})\right|_{\vec{\nu}=\frac{\vec{u}_{2}-\vec{u}_{1}}{\lambda d}}
\end{aligned}
$$

where $W(\vec{\nu})$ is the spatial spectrum of the weighting function $w(\vec{v})$ evaluated at frequency $\vec{\nu}$. With circular transmitter aperture $\mathcal{A}$ and circle binary weighting function at the receiver region $\mathcal{R}$, the optimal beam is proven by Slepian $[69,68]$ to be the Prolate spheroidal function, which is the principle eigenfunction of $\mathcal{H}_{0}\left(\vec{u}_{1}, \vec{u}_{2}\right)$ associated with the largest eigenvalue. A plot of $\mathcal{H}_{0}\left(u, u^{\prime}\right)$ is shown in Figure 4.3 for one dimensional propagation with rectangular weighting function.

\subsection{Optimal Beam to Maximize the Average Integrated Intensity}

When a beam propagates through random media such as the atmospheric turbulence, the impulse response $h(\vec{v}, \vec{u})$ and the kernel $\mathcal{H}\left(\vec{u}_{1}, \vec{u}_{2}\right)$ are random. 


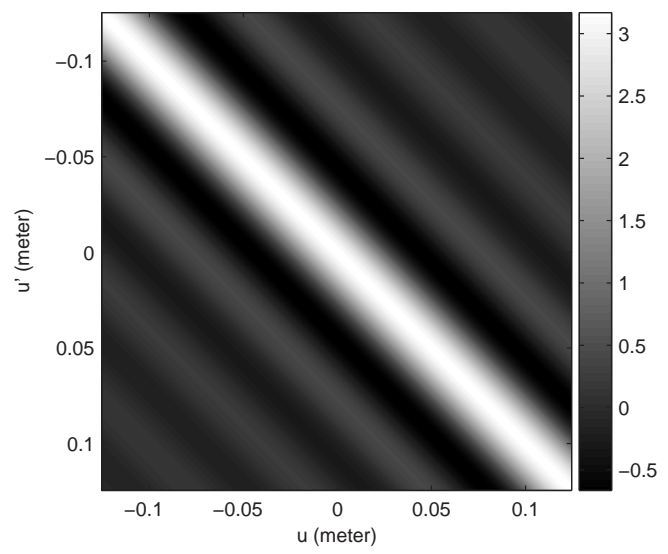

Figure 4.3: Kernel $\mathcal{H}_{0}\left(u, u^{\prime}\right)$ for one dimensional propagation in free space with rectangular weighting function.

Accordingly, eigenvalues and eigenfunctions of the kernel are subject to random perturbations. To evaluate the turbulence effects on the optimal beam, statistical properties of the eigenvalues and eigenfunctions of the perturbed kernel $\mathcal{H}\left(\vec{u}_{1}, \vec{u}_{2}\right)$ are needed. Unfortunately, it is not easy to get analytic results of these statistical properties for a randomly perturbed kernel. To overcome the difficulties existing in the above problem, we investigate the optimal beams that can compensate the beam spread statistically within a long exposure time.

In the case of propagation in random media, the average weighted intensity over turbulence at the target plane can be shown to be:

$$
\langle\mathcal{I}\rangle=\iint f^{*}\left(\vec{u}_{1}\right)\left\langle\mathcal{H}\left(\vec{u}_{1}, \vec{u}_{2}\right)\right\rangle f\left(\vec{u}_{2}\right) \mathrm{d} \vec{u}_{1} \mathrm{~d} \vec{u}_{2}
$$


where $\left\langle\mathcal{H}\left(\vec{u}_{1}, \vec{u}_{2}\right)\right\rangle$ is the averaged kernel over turbulence:

$$
\left\langle\mathcal{H}\left(\vec{u}_{1}, \vec{u}_{2}\right)\right\rangle=a^{*}\left(\vec{u}_{1}\right) \int w(\vec{v})\left\langle h^{*}\left(\vec{v}, \vec{u}_{1}\right) h\left(\vec{v}, \vec{u}_{2}\right)\right\rangle \mathrm{d} \vec{v} a\left(\vec{u}_{2}\right)
$$

Then the optimal beam can be defined to be the transmitted field that maximizes the average integrated intensity $\langle\mathcal{I}\rangle$ :

$$
\hat{f}(\vec{u})=\arg \max _{\|f\|^{2}=\mathcal{I}_{0}}\langle\mathcal{I}\rangle
$$

It is evident that the optimal beam is the largest eigenvalue's eigenfunction for the averaged Hermitian kernel $\left\langle\mathcal{H}\left(\vec{u}_{1}, \vec{u}_{2}\right)\right\rangle$. The eigenvalues and eigenvectors of $\left\langle\mathcal{H}\left(\vec{u}_{1}, \vec{u}_{2}\right)\right\rangle$ depend on the statistical properties of the random media.

\subsection{Averaged Kernel in Turbulence}

It is easy to see that the averaged kernel $\left\langle\mathcal{H}\left(\vec{u}_{1}, \vec{u}_{2}\right)\right\rangle$ over turbulence is important in this problem. In this section, we will discuss how the kernel $\mathcal{H}\left(\vec{u}_{1}, \vec{u}_{2}\right)$ has been changed in turbulence and the corresponding effects on its eigenvalues and eigenvectors.

\subsubsection{Averaged kernel for the phase screen model}

Consider the geometry of a thin turbulence layer near the pupil and take the standard assumption that the refractive index is a Gaussian random process. The optical length variations cause Gaussian phase perturbations at the pupil 
plane as

$$
\phi(\vec{u})=k \int_{z}^{z+\Delta z} n(\vec{u}, z) \mathrm{d} z,
$$

where $k$ is the optical wave number and $n(\vec{u}, z)$ is the index of refraction variation as a function of positions. The transmittance of such a phase screen takes the form

$$
t_{s}(\vec{u})=\exp [j \phi(\vec{u})]
$$

where the random phase shift $\phi(\vec{u})$ introduced at position $\vec{u}$ is assumed to be wide sense stationary (WSS). The averaged kernel in this case follows

$$
\left\langle\mathcal{H}\left(\vec{u}_{1}, \vec{u}_{2}\right)\right\rangle=\mathcal{H}_{0}\left(\vec{u}_{1}, \vec{u}_{2}\right)\left\langle e^{j \Delta \phi\left(\vec{u}_{1}, \vec{u}_{2}\right)}\right\rangle,
$$

where $\mathcal{H}_{0}\left(\vec{u}_{1}, \vec{u}_{2}\right)$ is the free space kernel and

$$
\Delta \phi\left(\vec{u}_{1}, \vec{u}_{2}\right)=\phi\left(\vec{u}_{1}\right)-\phi\left(\vec{u}_{2}\right)
$$

is the phase difference between two positions $\vec{u}_{1}$ and $\vec{u}_{2}$ at the pupil plane. The random phase $\phi(\vec{u})$ can be modeled as a zero mean Gaussian random process [58]. Then the phase difference $\Delta \phi\left(\vec{u}_{1}, \vec{u}_{2}\right)$ is also a zero mean Gaussian process with following statistical properties

$$
\left\langle\Delta \phi\left(\vec{u}_{1}, \vec{u}_{2}\right)\right\rangle=0
$$

and

$$
\begin{aligned}
\sigma_{\Delta \phi}^{2} & =\left\langle\left[\phi\left(\vec{u}_{1}\right)-\phi\left(\vec{u}_{2}\right)\right]^{2}\right\rangle \\
& =D_{\phi}\left(\vec{u}_{1}, \vec{u}_{2}\right)
\end{aligned}
$$


where $D_{\phi}\left(\vec{u}_{1}, \vec{u}_{2}\right)$ is the structure function of the random process $\phi(\vec{u})$. For Gaussian random phase, we have

$$
\left\langle e^{j \Delta \phi\left(\vec{u}_{1}, \vec{u}_{2}\right)}\right\rangle=\exp \left[-\frac{1}{2} D_{\phi}\left(\vec{u}_{1}, \vec{u}_{2}\right)\right]
$$

By using the Kolmogorov power spectral density to characterize the index of refraction fluctuations, the structure function takes the form

$$
D_{\phi}\left(\vec{u}_{1}, \vec{u}_{2}\right)=6.88\left(\frac{\left\|\vec{u}_{1}-\vec{u}_{2}\right\|}{r_{0}}\right)^{5 / 3}
$$

where $r_{0}$ is the Fried parameter of the turbulence [58]. So the averaged kernel takes the form

$$
\begin{aligned}
\left\langle\mathcal{H}\left(\vec{u}_{1}, \vec{u}_{2}\right)\right\rangle & =\mathcal{H}_{0}\left(\vec{u}_{1}, \vec{u}_{2}\right)\left\langle e^{j \Delta \phi\left(\vec{u}_{1}, \vec{u}_{2}\right)}\right\rangle \\
& =\frac{e^{-j \frac{\pi}{\lambda d}\left(\left\|\vec{u}_{1}\right\|^{2}-\left\|\vec{u}_{2}\right\|^{2}\right)}}{(\lambda d)^{2}} a^{*}\left(\vec{u}_{1}\right) a\left(\vec{u}_{2}\right) W\left(\frac{\vec{u}_{1}-\vec{u}_{2}}{\lambda d}\right) e^{-\frac{1}{2} D_{\phi}\left(\vec{u}_{1}-\vec{u}_{2}\right)}
\end{aligned}
$$

The principle eigenvector of this kernel is the optimal beam to maximize $\langle\mathcal{I}\rangle$. An image of the averaged kernel $\left\langle\mathcal{H}\left(u, u^{\prime}\right)\right\rangle$ for one dimensional propagation is shown in Figure 4.4 .

Notice that both the structure function $D_{\phi}\left(\vec{u}_{1}, \vec{u}_{2}\right)$ and the spectrum $W\left(\vec{u}_{1}, \vec{u}_{2}\right)$ are functions of $\Delta u=\left\|\vec{u}_{1}-\vec{u}_{2}\right\|$. Therefore, we define a new weighting function in the frequency domain as

$$
W_{n}\left(\frac{\vec{u}_{1}-\vec{u}_{2}}{\lambda d}\right)=\exp \left[-\frac{1}{2} D_{\phi}\left(\vec{u}_{1}-\vec{u}_{2}\right)\right] W\left(\frac{\vec{u}_{1}-\vec{u}_{2}}{\lambda d}\right) .
$$

Then we use the inverse Fourier transform to obtain the new weighting func- 


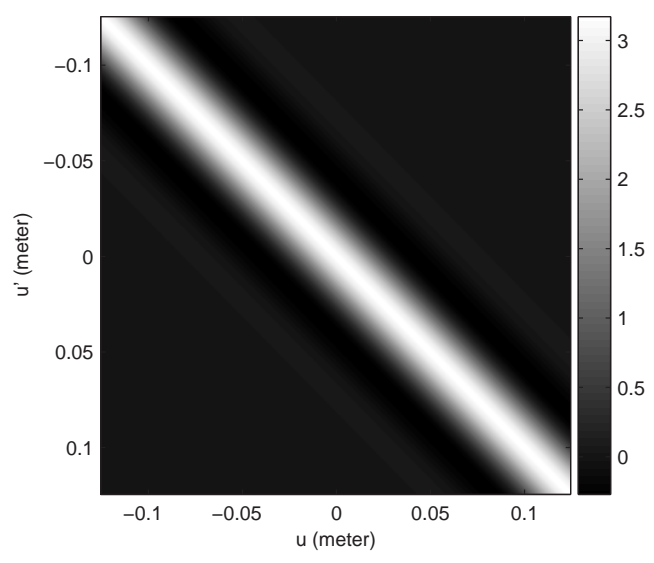

Figure 4.4: Averaged kernel $\left\langle\mathcal{H}\left(u, u^{\prime}\right)\right\rangle$ for one dimensional propagation through turbulence with rectangular weighting function and $r_{0}=10 \mathrm{~cm}$.

tion in the space domain

$$
w_{n}(\vec{v})=\mathcal{F}^{-1}\left\{\exp \left[-\frac{1}{2} D_{\phi}\left(\vec{u}_{1}-\vec{u}_{2}\right)\right] W\left(\frac{\vec{u}_{1}-\vec{u}_{2}}{\lambda d}\right)\right\}
$$

The averaged kernel can then be expressed as

$$
\left\langle\mathcal{H}\left(\vec{u}_{1}, \vec{u}_{2}\right)\right\rangle=\frac{e^{-j \frac{\pi}{\lambda d}\left(\left\|\vec{u}_{1}\right\|^{2}-\left\|\vec{u}_{2}\right\|^{2}\right)}}{(\lambda d)^{2}} a^{*}\left(\vec{u}_{1}\right) a\left(\vec{u}_{2}\right) W_{n}\left(\frac{\vec{u}_{1}-\vec{u}_{2}}{\lambda d}\right) .
$$

and the average integrated intensity can be expressed as

$$
\langle\mathcal{I}\rangle=\int w_{n}(\vec{v})|g(\vec{v})|^{2} \mathrm{~d} \vec{v}
$$

where $g(\vec{v})$ is the received field in free space. An example of the changes of weighting function is shown in Figure 4.5.

Equation (4.32) indicates that the average integrated intensity weighted by $w(\vec{v})$ at target through turbulence equals the integrated intensity through free space weighted by another function $w_{n}(\vec{v})$. So the optimal beam with 


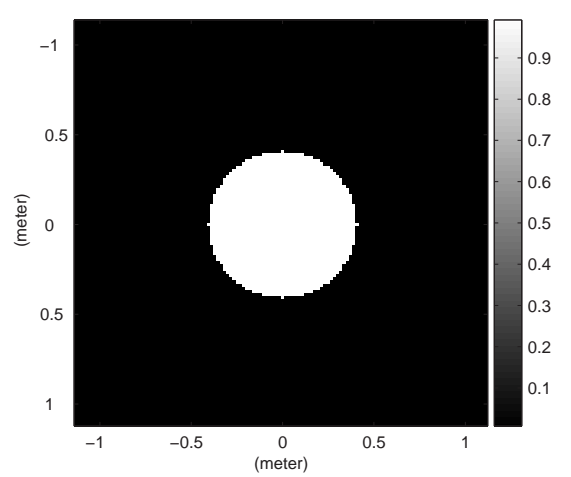

(a)

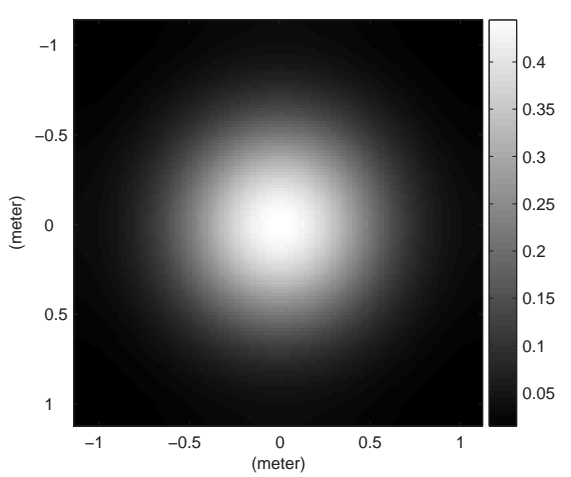

(b)

Figure 4.5: Weighting function changes: (a) Original weighting function. (b) New weighting function in turbulence.

this new weighting function in free space should also be the optimal beam to maximize the average integrated intensity through turbulence with the original weighting function $w(\vec{v})$.

It is easy to see qualitatively from this new weighting function how much power is lost inside the receiver due to turbulence compared with free space propagation. The definition of $w_{n}(\vec{v})$ has also been used in the numerical computation of the optimal beam to simplify the simulation complexity in this study. By using $w_{n}(\vec{v})$, there is no more need to generate random phase screens and propagate optical beams through them in the iterative algorithm.

\subsubsection{Averaged kernel for the extended Huygens-Fresnel principle model}

From Section 3.5.4 we know that according to the extended Huygens-Fresnel principle, the impulse function of propagation through turbulence takes the 
form

$$
h(\vec{v}, \vec{u})=\frac{1}{j \lambda d} \exp \left(j \frac{2 \pi}{\lambda} d\right) \exp \left(j \frac{\pi}{\lambda d}\|\vec{v}-\vec{u}\|^{2}+\psi(\vec{v}, \vec{u})\right)
$$

where $\psi(\vec{v}, \vec{u})$ is the random part of the complex phase. So the averaged kernel becomes

$$
\begin{aligned}
\left\langle\mathcal{H}\left(\vec{u}_{1}, \vec{u}_{2}\right)\right\rangle= & a^{*}\left(\vec{u}_{1}\right) \int w(\vec{v})\left\langle h^{*}\left(\vec{v}, \vec{u}_{1}\right) h\left(\vec{v}, \vec{u}_{2}\right)\right\rangle \mathrm{d} \vec{v} a\left(\vec{u}_{2}\right) \\
= & a^{*}\left(\vec{u}_{1}\right) a\left(\vec{u}_{2}\right) \frac{1}{(\lambda d)^{2}} e^{-j \frac{\pi}{\lambda d}\left(\left\|\vec{u}_{1}\right\|^{2}-\left\|\vec{u}_{2}\right\|^{2}\right)} \\
& \times \int w(\vec{v}) e^{j \frac{2 \pi}{\lambda d} \cdot\left(\vec{u}_{1}-\vec{u}_{2}\right)}\left\langle\exp \left[\psi^{*}\left(\vec{v}, \vec{u}_{1}\right)+\psi\left(\vec{v}, \vec{u}_{2}\right)\right]\right\rangle \mathrm{d} \vec{v} .
\end{aligned}
$$

The second order moment of the extended Huygens Fresnel principle had been studied in [82] and the modulation transfer function (MTF) of the turbulence was shown to be

$$
\begin{aligned}
M\left(\vec{v}_{1}, \vec{v}_{2}, \vec{u}_{1}, \vec{u}_{2}\right) & =\left\langle\exp \left[\psi\left(\vec{v}_{1}, \vec{u}_{1}\right)+\psi^{*}\left(\vec{v}_{2}, \vec{u}_{2}\right)\right]\right\rangle \\
& =\exp \left\{-\left[\left\langle\left|\psi_{1}\right|^{2}\right\rangle-\left\langle\psi_{1}\left(\vec{v}_{1}, \vec{u}_{1}\right) \psi_{1}^{*}\left(\vec{v}_{2}, \vec{u}_{2}\right)\right\rangle\right]\right\}
\end{aligned}
$$

where $\psi_{1}=f_{1} / f_{0}, f_{0}$ is the field in the absence of turbulence and $f_{1}$ is the first order Born approximation, and

$$
\left\langle\left|\psi_{1}\right|^{2}\right\rangle=2 \pi k^{2} d \int \Phi_{n}(\vec{\kappa}) \mathrm{d} \vec{\kappa}
$$

This expression is valid through terms of the second order in refractive index perturbation $n_{s}$. Experimental evidence and theoretical arguments indicate that $\psi$ is a Gaussian random variable, which means that the above 
equation is correct to all orders in $n_{s}$. For a spherical wave with paraxial approximation and local homogeneous random media, Yura [82] shows that

$$
\left\langle\psi_{1}\left(\vec{v}_{1}, \vec{u}_{1}\right) \psi_{1}^{*}\left(\vec{v}_{2}, \vec{u}_{2}\right)\right\rangle=4 \pi^{2} k^{2} d \int \vec{\kappa} \Phi_{n}(\vec{\kappa}) \mathrm{d} \vec{\kappa} \int_{0}^{1} J_{0}[|t \Delta v+(1-t) \Delta u| \vec{\kappa}] \mathrm{d} t
$$

where $J_{0}$ is the first kind of Bessel function of zero order and

$$
\Delta u=\left\|\vec{u}_{1}-\vec{u}_{2}\right\|, \quad \Delta v=\left\|\vec{v}_{1}-\vec{v}_{2}\right\|
$$

Then the MTF can be expressed as

$$
\begin{aligned}
M(\Delta v, \Delta u) & =\exp \left\{-\left[\left\langle\left|\psi_{1}\right|^{2}\right\rangle-\left\langle\psi_{1}\left(\vec{v}, \vec{u}_{1}\right) \psi_{1}^{*}\left(\vec{v}, \vec{u}_{2}\right)\right\rangle\right]\right\} \\
& =\exp \left\{-4 \pi^{2} k^{2} d \int_{0}^{1} \mathrm{~d} t \int \vec{\kappa} \Phi_{n}(\vec{\kappa})\left[1-J_{0}(|t \Delta v+(1-t) \Delta u| \vec{\kappa})\right] \mathrm{d} \vec{\kappa}\right\}
\end{aligned}
$$

For Kolmogorov spectrum characterized turbulence with $\Delta v \ll L_{0}$, it has been shown that [82]

$$
\begin{aligned}
M(\Delta v, \Delta u) & =\exp \left[-\frac{2.91}{2} C_{n}^{2} k^{2} d \int_{0}^{1} \mathrm{~d} t|t \Delta v+(1-t) \Delta u|^{5 / 3}\right] \\
& =\exp \left\{-\frac{2.91}{2} C_{n}^{2} k^{2} d\left[\left(\frac{3}{8}\right) \frac{\left(\Delta v^{8 / 3}-\Delta u^{8 / 3}\right)}{|\Delta v-\Delta u|}\right]\right\} .
\end{aligned}
$$

In the case of intensity fluctuations of a point at the target $\Delta v=0$, this equation becomes

$$
M(\Delta v=0, \Delta u)=\exp \left(-0.545 C_{n}^{2} k^{2} d \Delta u^{5 / 3}\right) .
$$


The transverse coherence length has been defined as

$$
\rho_{0}=\left(0.545 k^{2} C_{n}^{2} d\right)^{-3 / 5}
$$

so that

$$
M(\Delta v=0, \Delta u)=\exp \left[-\left(\frac{\Delta u}{\rho_{0}}\right)^{5 / 3}\right] .
$$

Finally, we get the averaged kernel for the extended Huygens-Fresnel propagation as

$$
\begin{aligned}
\left\langle\mathcal{H}\left(\vec{u}_{1}, \vec{u}_{2}\right)\right\rangle= & \mathcal{H}_{0}\left(\vec{u}_{1}, \vec{u}_{2}\right) \exp \left[-\left(\frac{\Delta u}{\rho_{0}}\right)^{5 / 3}\right] \\
= & a^{*}\left(\vec{u}_{1}\right) a\left(\vec{u}_{2}\right) \frac{1}{(\lambda d)^{2}} e^{-j \frac{\pi}{\lambda d}\left(\left\|\vec{u}_{1}\right\|^{2}-\left\|\vec{u}_{2}\right\|^{2}\right)} \\
& \times \int w(\vec{v}) \exp \left[j \frac{2 \pi}{\lambda d} \vec{v} \cdot\left(\vec{u}_{1}-\vec{u}_{2}\right)\right] \exp \left[-\left(\frac{\Delta u}{\rho_{0}}\right)^{5 / 3}\right] \mathrm{d} \vec{v} .
\end{aligned}
$$

Notice that the averaged kernels in different propagation models have very similar form. The extended Huygens-Fresnel principle includes random effects from both the transmitter and receiver sides for strong turbulence cases. But the second order moment of the field perturbation in this model does not differ much from the one in the phase screen model. This is because only perturbations in the far field have evident effects on the second order moment of the received perturbation. Another reason is that, because of the homogeneous and isotropic random media assumption, receiver region effects vanish when $\vec{v}_{1}=\vec{v}_{2}$. So when we deal with the second order moment of the field perturbation in $\left\langle\mathcal{H}\left(\vec{u}_{1}, \vec{u}_{2}\right)\right\rangle$, we get similar results for the extended Huygens-Fresnel principle and the phase screen model. 
This result is not unexpected because most of the intensity fluctuations are induced by turbulence far from the receiver aperture. Near aperture turbulence contributes mainly to the phase distortions but not the field amplitude $(\Delta v=0)$. So the average kernel $\left\langle H\left(\vec{u}_{1}, \vec{u}_{2}\right)\right\rangle$ is affected mainly by the second order statistics of turbulence far from the receiver.

The MTF of propagation in the extended Huygens-Fresnel principle model has also been expressed in the structure function form as $[6,24]$

$$
\begin{aligned}
M(\Delta v, \Delta u) & =\left\langle\exp \left[\psi^{*}\left(\vec{v}_{1}, \vec{u}_{1}\right)+\psi\left(\vec{v}_{2}, \vec{u}_{2}\right)\right]\right\rangle \\
& =\exp \left[-D_{\psi}(\Delta v, \Delta u) / 2\right]
\end{aligned}
$$

where

$$
D_{\psi}(\Delta v, \Delta u)=2.91 C_{n}^{2} k^{2} d\left[\left(\frac{3}{8}\right) \frac{\left(\Delta v^{8 / 3}-\Delta u^{8 / 3}\right)}{|\Delta v-\Delta u|}\right]
$$

is the structure function of the complex phase $\psi(\vec{v}, \vec{u})$. We will use this expression later to be consistent with the discussion in random phase screen model.

\subsection{Performance of the Optimal Beams}

To maximize the average integrated intensity, the optimal beam is the largest eigenvalue's eigenvector of $\left\langle\mathcal{H}\left(\vec{u}_{1}, \vec{u}_{2}\right)\right\rangle$, which establishes an upper bound for the performance of different compensation schemes. We will compute the optimal beams and the corresponding maximum integrated intensities for different propagation cases in this section. Because the two turbulence 
models - extended Heygens-Fresnel principle and phase screen model - have similar results on the second moment of the received field, we will use the Fried parameter to characterize the turbulence strength in our experiments.

\subsubsection{One dimensional experiment}

We begin with the optimal beam in the one dimensional case because of computational simplicity. To evaluate the performance of the optimal beams, we introduce one dimensional free space propagation first. Consider the Fresnel diffraction from a one dimensional rectangular slit aperture $\mathcal{A}$,

$$
a(u)=\left\{\begin{array}{ll}
1 & |u| \leqslant D / 2 \\
0, & |u|>D / 2
\end{array},\right.
$$

where $D$ is the width of the slit. The free space received field at distance $d$ can be expressed as

$$
g(v)=\int_{\mathcal{A}} h(v, u) f(u) \mathrm{d} u
$$

where

$$
h(v, u)=\frac{1}{\sqrt{j \lambda d}} \exp \left(j \frac{2 \pi}{\lambda} d\right) \exp \left(j \frac{\pi}{\lambda d}|v-u|^{2}\right)
$$

is the impulse function of one dimensional Fresnel propagation. When the focused beam is transmitted from the pupil,

$$
f(u)=\exp \left(-j \frac{\pi}{\lambda d}|u|^{2}\right)
$$


the received intensity is

$$
\begin{aligned}
|g(v)|^{2} & =\frac{1}{\lambda d}\left|\int a(u) \exp \left(-j \frac{2 \pi}{\lambda d} u v\right)\right|^{2} \\
& =\frac{1}{\lambda d}\left|A\left(\frac{v}{\lambda d}\right)\right|^{2}
\end{aligned}
$$

where $A(\nu)$ is the Fourier transform of the aperture function $a(u)$

$$
A(\nu)=D \operatorname{sinc}(D \nu)
$$

The sinc function in the above equation is defined as

$$
\operatorname{sinc}(u)=\left\{\begin{array}{ll}
\frac{\sin (\pi u)}{\pi u} & u \neq 0 \\
1 & u=0
\end{array} .\right.
$$

For a rectangular receiver region $\mathcal{R}$ :

$$
w(v)= \begin{cases}1 & |v| \leqslant r \\ 0 & |v|>r\end{cases}
$$

and the integrated intensity can be derived as

$$
\begin{aligned}
\mathcal{I} & =\int w(v)|g(v)|^{2} \mathrm{~d} v \\
& =\frac{2 D^{2}}{\lambda d} \int_{0}^{r} \operatorname{sinc}^{2}\left(\frac{D v}{\lambda d}\right) \mathrm{d} v .
\end{aligned}
$$

As mentioned before, the optimal beams in free space are the prolate functions. In the case of one dimensional propagation, we can compute the optimal beams and integrated intensities through the eigen decomposition of the kernel $\mathcal{H}\left(u_{1}, u_{2}\right)$ directly. A plot of the optimal beams and correspond- 


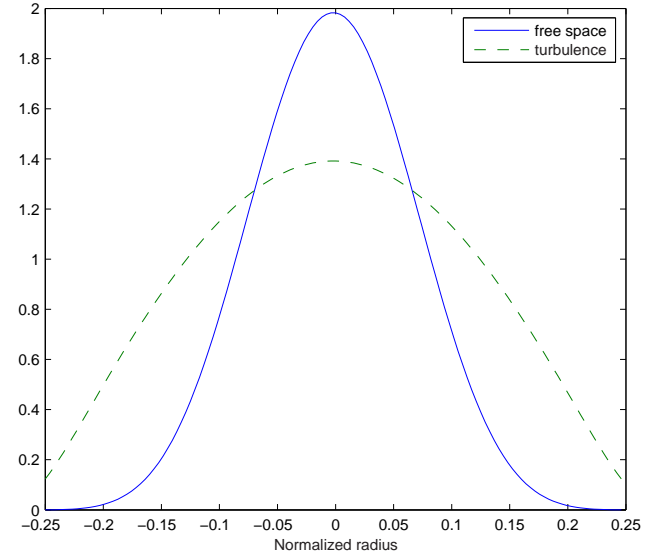

(a)

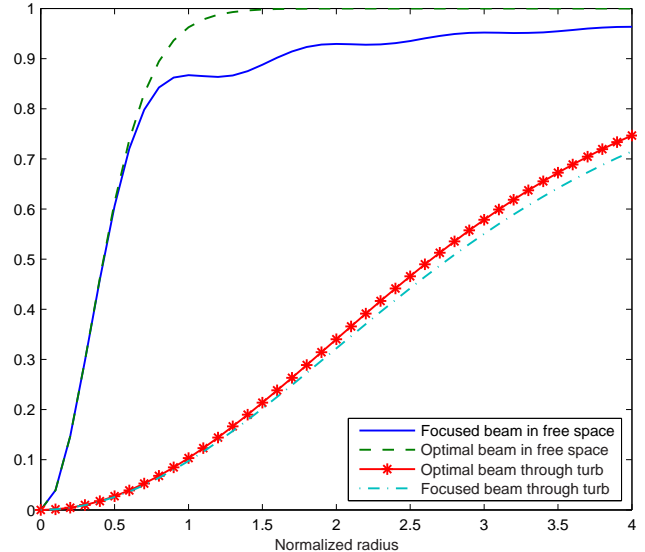

(b)

Figure 4.6: Optimal beams and integrated intensity for one dimensional propagation in free space and random medium: (a) Optimal beam $\hat{f}(u)$ at transmitter aperture, (b) Fractional integrated intensity $\mathcal{I} / \mathcal{I}_{0}$ vs. receiver plane normalized radius $(r D) /(\lambda d)$.

ing integrated intensities of a one dimensional propagation experiment are shown in Figure 4.6. The physical parameters used in this one dimensional propagation experiment are shown in Table 4.1.

\begin{tabular}{cccl}
\hline \hline Physical parameter & Symbol & \multicolumn{2}{c}{ Value } \\
\hline wave length & $\lambda$ & 1.0 & $\mu \mathrm{m}$ \\
propagation distance & $\mathrm{d}$ & 20 & $\mathrm{~km}$ \\
transmitter diameter & $D$ & 0.5 & $\mathrm{~m}$ \\
Fried parameter & $r_{0}$ & 10 & $\mathrm{~cm}$ \\
\hline \hline
\end{tabular}

Table 4.1: Physical parameters used in the one dimensional propagation simulation. 


\subsubsection{Two dimensional experiment}

As we have shown, an Airy pattern is formed at the target for a focused beam transmitted from a circular aperture in free space. In the two dimensional case, it is difficult to do direct eigen decomposition of the four dimensional Hermitian kernel $\mathcal{H}\left(\vec{u}_{1}, \vec{u}_{2}\right)$. To compute the principle eigen beam of $\mathcal{H}\left(\vec{u}_{1}, \vec{u}_{2}\right)$, an iterative algorithm developed by Schulz [62] is used here. In the propagation through random medium, a new weighting function $w_{n}(\vec{v})$ are used in this algorithm and Monte-Carlo method is avoided. We set up the two dimensional propagation experiment by using similar parameters as in the one dimensional case, with aperture diameter $D=0.4 \mathrm{~m}$ and propagation distance $d=20 \mathrm{~km}$. Plots of the optimal beams and the average integrated intensities in free space and through turbulence are shown in Figure 4.7.

\subsubsection{Performance analysis}

We have seen that experiments in the one dimensional and two dimensional cases show very similar results. In free space propagation, the focused beam performs well with small target radius and the optimal beam makes improvements with large radius. In the case of propagation through random media, the average integrated intensity of the optimal beam through turbulence does not have evident improvement compared with other beams, which means that there is not much improvement space for the optical system. This can be explained from the kernel changes we have shown before.

Compare the averaged kernel in Figure 4.4 with the one in Figure 4.3 


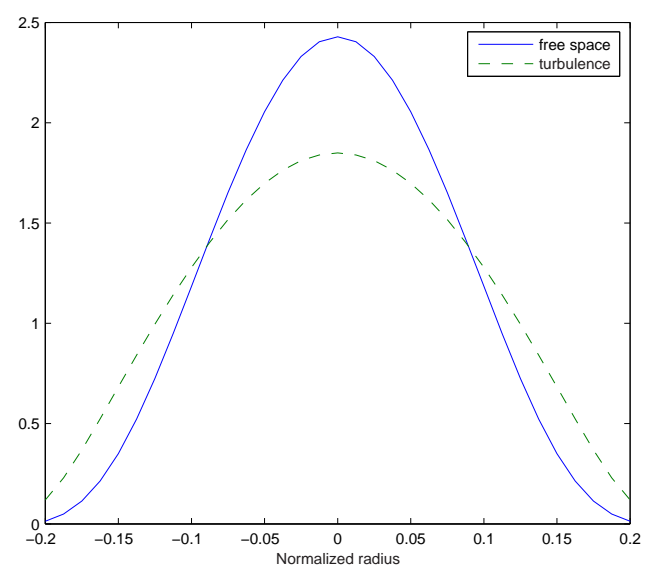

(a)

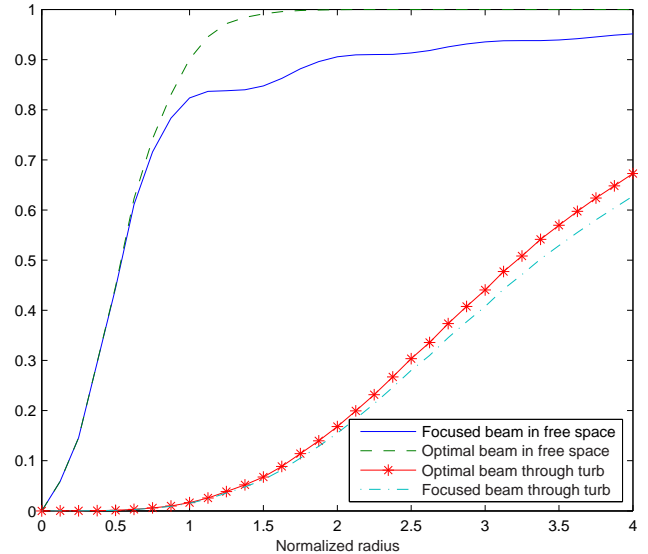

(b)

Figure 4.7: Optimal beams and integrated intensity for two dimensional propagation in free space and random medium: (a) A center slice of the optimal beam $\hat{f}(\vec{u})$ at transmitter aperture, (b) Fractional integrated intensity $\mathcal{I} / \mathcal{I}_{0}$ vs. receiver plane normalized radius $(r D) /(\lambda d)$ for focused beam and optimal beam.

for free space, we can see easily that the averaged kernel after turbulence is more like the identity matrix. This explains intuitively why the optimal beam does not improve the integrated intensity greatly. For the identity matrix, any vector can be its eigenvector with all the same eigenvalues equal to 1 . So for $\left\langle\mathcal{H}\left(\vec{u}_{1}, \vec{u}_{2}\right)\right\rangle$, which is close to the identity matrix, its eigenvalues are close in value. In our consideration, because the summation of all the eigenvalues of the kernel $\mathcal{H}\left(\vec{u}_{1}, \vec{u}_{2}\right)$ indicates the whole transmitted energy/power, evenly distributed eigenvalues implies that all the eigenvectors have nearly the same performance. So there is no beam that can perform much better than others.

This result indicates that, to compensate the average integrated intensity by changing the transmitted field does not work well since there is not much 
improvement space for it. Short term compensation schemes, compensating over a short exposure time, is preferred to maximize the integrated intensity in real time. Adaptive optics can be used to do the compensation in the pupil or the image plane. 


\section{Chapter 5}

\section{Optimal Beam to Minimize the Scintillation Index}

Laser beam scintillation is an important random-media effect that degrades

optical system performance. The scintillation index is commonly used to measure the severity of the disturbance. Whereas fully coherent beams are extremely sensitive to the random media, recent investigations show that partially coherent beams can be less sensitive to the random media [28, 56, 65]. In [63], the optimal beam to minimize the scintillation index has been proven to be partially coherent in general cases. The coherent modes and associated integrated intensities depend on the second and forth order moments of the propagation Green's function $h(\vec{v}, \vec{u})$. 


\subsection{Optimal Pupil Plane Mutual Coherence}

As shown in equation (3.104), the scintillation index has been defined as the normalized variance of the received integrated intensity

$$
S_{\mathcal{I}}=\frac{\sigma_{\mathcal{I}}^{2}}{\left(\mu_{\mathcal{I}}\right)^{2}}=\frac{\left\langle\mathcal{I}^{2}\right\rangle}{\langle\mathcal{I}\rangle^{2}}-1,
$$

where $\langle\cdot\rangle$ is the average over turbulence and $\mathcal{I}$ denotes the integrated intensity at the target plane. Consider the propagation of a source field from aperture $\mathcal{A}$ to receiver region $\mathcal{R}$. The (short term average) intensity of the received field at $\mathcal{R}$ can be expressed as

$$
i(\vec{v})=\iint_{\mathcal{A}^{2}} h^{*}\left(\vec{v}, \vec{u}_{1}\right) J\left(\vec{u}_{1}, \vec{u}_{2}\right) h\left(\vec{v}, \vec{u}_{2}\right) \mathrm{d} \vec{u}_{1} \mathrm{~d} \vec{u}_{2}
$$

where $J\left(\vec{u}_{1}, \vec{u}_{2}\right)=\left\langle f^{*}\left(\vec{u}_{1}\right) f\left(\vec{u}_{2}\right)\right\rangle$ is the mutual intensity of the transmitted field in the pupil plane. According to the analysis in section 3.3.3, $J\left(\vec{u}_{1}, \vec{u}_{2}\right)$ has the coherent mode expansion in the aperture region as

$$
J\left(\vec{u}_{1}, \vec{u}_{2}\right)=\sum_{k} \alpha_{k} \psi_{k}\left(\vec{u}_{1}\right) \psi_{k}^{*}\left(\vec{u}_{2}\right), \quad\left(\vec{u}_{1}, \vec{u}_{2}\right) \in \mathcal{A}^{2},
$$

where $\Psi=\left\{\psi_{k}(\vec{u})\right\}$ are the orthonormal coherent modes over aperture region $\mathcal{A}$, and $\left\{\alpha_{k}\right\}$ are nonnegative real numbers associated with the integrated intensity of single modes. When a single coherent mode $\psi_{k}(\vec{u})$ propagates to the target, the received field is

$$
\zeta_{k}(\vec{v})=\int_{\mathcal{A}} h(\vec{v}, \vec{u}) \psi_{k}(\vec{u}) \mathrm{d} \vec{u}
$$


and the corresponding received integrated intensity of this single mode is

$$
\mathcal{I}_{k}=\int w(\vec{v})\left|\zeta_{k}(\vec{v})\right|^{2} \mathrm{~d} \vec{v}=\int w(\vec{v})\left|\int_{\mathcal{A}} h(\vec{v}, \vec{u}) \psi_{k}(\vec{u}) \mathrm{d} \vec{u}\right|^{2} \mathrm{~d} \vec{v} .
$$

Because $\psi_{k}(\vec{v})$ is normalized, $0 \leqslant \mathcal{I}_{k} \leqslant 1$. The total received intensity from the optical source with mutual intensity $J\left(\vec{u}_{1}, \vec{u}_{2}\right)$ is

$$
\begin{aligned}
\mathcal{I} & =\int w(\vec{v}) \iint_{\mathcal{A}^{2}} h^{*}\left(\vec{v}, \vec{u}_{1}\right) J\left(\vec{u}_{1}, \vec{u}_{2}\right) h\left(\vec{v}, \vec{u}_{2}\right) \mathrm{d} \vec{u}_{1} \mathrm{~d} \vec{u}_{2} \mathrm{~d} \vec{v} \\
& =\sum_{k} \alpha_{k} \int w(\vec{v})\left|\zeta_{k}(\vec{v})\right|^{2} \mathrm{~d} \vec{v}=\sum_{k} \alpha_{k} \mathcal{I}_{k} .
\end{aligned}
$$

The statistical properties of the received intensities $\left\{\mathcal{I}_{k}\right\}$ are important in deriving the statistics of the total received intensity $\mathcal{I}$. The average received intensity of a single transmitted mode can be expressed as

$$
\mu_{k}=\left\langle\mathcal{I}_{k}\right\rangle=\iint_{\mathcal{A}^{2}} \psi_{k}^{*}\left(\vec{u}_{1}\right)\left\langle\mathcal{H}\left(\vec{u}_{1}, \vec{u}_{2}\right)\right\rangle \psi_{k}\left(\vec{u}_{2}\right) \mathrm{d} \vec{u}_{1} \mathrm{~d} \vec{u}_{2}
$$

where

$$
\left\langle\mathcal{H}\left(\vec{u}_{1}, \vec{u}_{2}\right)\right\rangle=\int w(\vec{v})\left\langle h^{*}\left(\vec{v}, \vec{u}_{1}\right) h\left(\vec{v}, \vec{u}_{2}\right)\right\rangle \mathrm{d} \vec{v}
$$

is the averaged kernel over turbulence. Then we can express the mean of the integrated intensity at the receiver as

$$
\langle\mathcal{I}\rangle=\sum_{k} \alpha_{k} \mu_{k}
$$

To calculate the scintillation index, the second order moments of the received 
intensity are needed:

$$
\left\langle\mathcal{I}^{2}\right\rangle-\langle\mathcal{I}\rangle^{2}=\sum_{k} \sum_{l} \alpha_{k} \mathrm{R}_{k l} \alpha_{l}
$$

where

$$
\mathrm{R}_{k l}=\left\langle\mathcal{I}_{k} \mathcal{I}_{l}\right\rangle-\mu_{k} \mu_{l}
$$

It has been shown in [63] that the optimal orthonormal coherence $\hat{\Psi}$ to minimize the scintillation index can be expressed as

$$
\hat{\Psi}=\arg \max \sum_{k} \sum_{l} \mu_{k}(\Psi) R_{k l}^{-1}(\Psi) \mu_{l}(\Psi),
$$

with associated integrated intensities $\hat{\alpha_{k}}$ as

$$
\hat{\alpha_{k}}=\sum_{l} R_{k l}^{-1}(\hat{\Psi}) \mu_{l}(\hat{\Psi})
$$

where $R_{k l}^{-1}$ is defined as:

$$
\sum_{l} R_{k l}^{-1}(\Psi) R_{l m}(\Psi)= \begin{cases}1 & k=m \\ 0 & k \neq l\end{cases}
$$

Notice that we have assumed all $\hat{\alpha_{k}}$ are non-negative here. In the case there are possible negative $\hat{\alpha_{k}}$ values, a constrained optimization is used to approach the optimal modes.

It can be seen from the above equations that the optimal pupil plane coherence to minimize the scintillation index depends on the first and second moments of the received intensity $\mathcal{I}$ through turbulence. Further discussion requires more information about the wave propagation in turbulence. In the 
following two sections, we will discuss the first and second moments of the integrated intensity in two different models: the pupil plane phase screen model and the extended Huygens-Fresnel model.

\subsection{Optimal Beams in Random Phase Screen Model}

Consider wave propagation for the pupil plane random phase screen model introduced in section 3.5.5. The received optical field can be expressed as

$$
f(\vec{v})=\int_{\mathcal{A}} h(\vec{v}, \vec{u}) f(\vec{u}) e^{j \phi(\vec{u})} \mathrm{d} \vec{u} .
$$

For Gaussian distributed random process $\phi(\vec{u})$, we have noted that the averaged kernel over turbulence takes the form

$$
\begin{aligned}
\left\langle\mathcal{H}\left(\vec{u}_{1}, \vec{u}_{2}\right)\right\rangle & =\mathcal{H}_{0}\left(\vec{u}_{1}, \vec{u}_{2}\right)\left\langle e^{j\left[\phi\left(\vec{u}_{1}\right)-\phi\left(\vec{u}_{2}\right)\right]}\right\rangle \\
& =\mathcal{H}_{0}\left(\vec{u}_{1}, \vec{u}_{2}\right) e^{-\frac{1}{2} D_{\phi}\left(\vec{u}_{1}, \vec{u}_{2}\right)}
\end{aligned}
$$

where $\mathcal{H}_{0}\left(\vec{u}_{1}, \vec{u}_{2}\right)$ is the kernel in free space, and $D_{\phi}$ is the phase structure function. For local stationary and isotropic random media with refractive index fluctuation characterized by the Kolmogorov power spectral density, we have shown before that

$$
D_{\phi}\left(\vec{u}_{1}, \vec{u}_{2}\right)=6.88\left(\frac{\left\|\vec{u}_{1}-\vec{u}_{2}\right\|}{r_{0}}\right)^{5 / 3},
$$

where $r_{0}$ is the Fried parameter. 


\subsubsection{First moment of the integrated intensity}

The average received intensity for transmitting a single coherent mode $\psi_{k}(\vec{u})$ can be derived as

$$
\begin{aligned}
\mu_{k} & =\left\langle\mathcal{I}_{k}\right\rangle=\iint_{\mathcal{A}^{2}} \psi_{k}^{*}\left(\vec{u}_{1}\right) \int w(\vec{v})\left\langle\mathcal{H}\left(\vec{u}_{1}, \vec{u}_{2}\right)\right\rangle \mathrm{d} \vec{v} \psi_{k}\left(\vec{u}_{2}\right) \mathrm{d} \vec{u}_{1} \mathrm{~d} \vec{u}_{2} \\
& =\left(\frac{1}{\lambda d}\right)^{2} \iint_{\mathcal{A}^{2}} \xi_{k}^{*}\left(\vec{u}_{1}\right) W\left(\frac{\vec{u}_{1}-\vec{u}_{2}}{\lambda d}\right) \exp \left[-\frac{1}{2} D_{\phi}\left(\vec{u}_{1}, \vec{u}_{2}\right)\right] \xi_{k}\left(\vec{u}_{2}\right) \mathrm{d} \vec{u}_{1} \mathrm{~d} \vec{u}_{2},
\end{aligned}
$$

where

$$
\xi_{k}(\vec{u})=\psi_{k}(\vec{u}) e^{j \frac{\pi}{\lambda d}\|\vec{u}\|^{2}} .
$$

As we have shown in Chapter $4, \mu_{k}$ depends on the second correlation of disturbed propagation Green's function.

\subsubsection{Second moment of the integrated intensity}

The second order moment of the integrated intensity at the receiver can be derived as

$$
\begin{aligned}
\left\langle\mathcal{I}_{k} \mathcal{I}_{l}\right\rangle= & \iiint \int_{\mathcal{A}^{4}} \psi_{k}^{*}\left(\vec{u}_{1}\right) \psi_{l}^{*}\left(\vec{u}_{3}\right) \psi_{k}\left(\vec{u}_{2}\right) \psi_{l}\left(\vec{u}_{4}\right)\left\langle\mathcal{H}\left(\vec{u}_{1}, \vec{u}_{2}\right) \mathcal{H}\left(\vec{u}_{3}, \vec{u}_{3}\right)\right\rangle \mathrm{d} \vec{u}_{1} \mathrm{~d} \vec{u}_{2} \mathrm{~d} \vec{u}_{3} \mathrm{~d} \vec{u}_{4} \\
= & \iiint \int_{\mathcal{A}^{4}} \psi_{k}^{*}\left(\vec{u}_{1}\right) \psi_{l}^{*}\left(\vec{u}_{3}\right) \psi_{k}\left(\vec{u}_{2}\right) \psi_{l}\left(\vec{u}_{4}\right) \mathcal{H}_{0}\left(\vec{u}_{1}, \vec{u}_{2}\right) \mathcal{H}_{0}\left(\vec{u}_{3}, \vec{u}_{4}\right) \\
& \times\left\langle\exp \left\{j\left[\phi\left(\vec{u}_{1}\right)-\phi\left(\vec{u}_{2}\right)+\phi\left(\vec{u}_{3}\right)-\phi\left(\vec{u}_{4}\right)\right]\right\}\right\rangle \mathrm{d} \vec{u}_{1} \mathrm{~d} \vec{u}_{2} \mathrm{~d} \vec{u}_{3} \mathrm{~d} \vec{u}_{4} \\
= & \left(\frac{1}{\lambda d}\right)^{4} \iiint \int_{\mathcal{A}^{4}} \xi_{k}^{*}\left(\vec{u}_{1}\right) \xi_{k}\left(\vec{u}_{2}\right) \xi_{l}^{*}\left(\vec{u}_{3}\right) \xi_{l}\left(\vec{u}_{4}\right) W\left(\frac{\vec{u}_{1}-\vec{u}_{2}}{\lambda d}\right) W\left(\frac{\vec{u}_{3}-\vec{u}_{4}}{\lambda d}\right) \\
& \times e^{\left[-\frac{D\left(\vec{u}_{1}-\vec{u}_{2}\right)}{2}-\frac{D\left(\vec{u}_{3}-\vec{u}_{4}\right)}{2}+\Gamma\left(\vec{u}_{1}-\vec{u}_{4}\right)+\Gamma\left(\vec{u}_{2}-\vec{u}_{3}\right)-\Gamma\left(\vec{u}_{1}-\vec{u}_{3}\right)-\Gamma\left(\vec{u}_{2}-\vec{u}_{4}\right)\right]} \mathrm{d} \vec{u}_{1} \mathrm{~d} \vec{u}_{2} \mathrm{~d} \vec{u}_{3} \mathrm{~d} \vec{u}_{4}
\end{aligned}
$$


where $\Gamma\left(\vec{u}_{1}, \vec{u}_{2}\right)=\left\langle\phi\left(\vec{u}_{1}\right) \phi\left(\vec{u}_{2}\right)\right\rangle$ is the autocorrelation function of random phase. For WSS Gaussian process $\phi(\vec{u})$ with zero mean, we have

$$
\Gamma\left(\vec{u}_{1}, \vec{u}_{2}\right)=\Gamma(0)-\frac{D\left(\vec{u}_{1}-\vec{u}_{2}\right)}{2} .
$$

Then we get

$$
\begin{aligned}
\left\langle\mathcal{I}_{k} \mathcal{I}_{l}\right\rangle= & \left(\frac{1}{\lambda d}\right)^{4} \iiint \int_{\mathcal{A}^{4}} \xi_{k}^{*}\left(\vec{u}_{1}\right) \xi_{l}^{*}\left(\vec{u}_{3}\right) \xi_{k}\left(\vec{u}_{2}\right) \xi_{l}\left(\vec{u}_{4}\right) W\left(\frac{\vec{u}_{1}-\vec{u}_{2}}{\lambda d}\right) W\left(\frac{\vec{u}_{3}-\vec{u}_{4}}{\lambda d}\right) \\
& \times e^{-\frac{1}{2}\left[D\left(\vec{u}_{1}-\vec{u}_{2}\right)+D\left(\vec{u}_{3}-\vec{u}_{4}\right)+D\left(\vec{u}_{1}-\vec{u}_{4}\right)+D\left(\vec{u}_{2}-\vec{u}_{3}\right)-D\left(\vec{u}_{1}-\vec{u}_{3}\right)-D\left(\vec{v}_{2}-\vec{u}_{4}\right)\right]} \mathrm{d} \vec{u}_{1} \mathrm{~d} \vec{u}_{2} \mathrm{~d} \vec{u}_{3} \mathrm{~d} \vec{u}_{4}
\end{aligned}
$$

The above equations about the first and second moments of the integrated intensity can be used to compute the optimal coherent mode set $\left(\boldsymbol{\Psi}=\left\{\psi_{k}(\vec{u})\right\}\right)$ and the associated weights $\left(\left\{\alpha_{k}\right\}\right)$ according to equation (5.12) and equation (5.13). The performance of the partially coherent source will be evaluated later.

\subsection{Optimal Beams in the extended Huygens- Fresnel principle model}

As we discussed in Section 3.5.4, the received field at distance $d$ under the extended Huygens-Fresnel principle can be expressed as

$$
g(\vec{v})=\frac{1}{j \lambda d} \exp \left(j \frac{2 \pi}{\lambda d}\right) \int_{\mathcal{A}} f(\vec{u}) \exp \left(j \frac{\pi}{\lambda d}\|\vec{v}-\vec{u}\|^{2}+\psi(\vec{v}, \vec{u})\right) \mathrm{d} \vec{u}
$$

where $\psi(\vec{v}, \vec{u})$ is the random part of the complex phase. Second and fourth order moments of this random part are required in the computation of the 
optimal pupil plane coherence. It was shown that the simulation results assuming Gaussian distribution of $\psi(\vec{v}, \vec{u})$ in this extended Huygens-Fresnel principle model agreed with experimental data [6].

\subsubsection{First moment of the integrated intensity}

The first moment of the received intensity after turbulence propagation can be expressed as

$$
\langle\mathcal{I}\rangle=\iint_{\mathcal{A}^{2}} f^{*}\left(\vec{u}_{1}\right)\left\langle\mathcal{H}\left(\vec{u}_{1}, \vec{u}_{2}\right)\right\rangle f\left(\vec{u}_{2}\right) \mathrm{d} \vec{u}_{1} \mathrm{~d} \vec{u}_{2}
$$

where the averaged kernel has been derived in Section 4.3.2 and takes the form

$$
\begin{aligned}
\left\langle\mathcal{H}\left(\vec{u}_{1}, \vec{u}_{2}\right)\right\rangle & =\mathcal{H}_{0}\left(\vec{u}_{1}, \vec{u}_{2}\right)\left\langle\exp \left[\psi^{*}\left(\vec{v}, \vec{u}_{1}\right)+\psi\left(\vec{v}, \vec{u}_{2}\right)\right]\right\rangle \\
& =\mathcal{H}_{0}\left(\vec{u}_{1}, \vec{u}_{2}\right) \exp \left[-\frac{1}{2} D_{\psi}(\Delta v, \Delta u)\right]
\end{aligned}
$$

In local homogeneous turbulence cases with Kolmogorov spectrum of the refractive index fluctuation, we have shown in section 4.3.2 that the structure function of complex phase $\psi(\vec{u}, \vec{u})$ takes the form

$$
D_{\psi}(\Delta v, \Delta u)=2.91 C_{n}^{2} k^{2} d\left[\left(\frac{3}{8}\right) \frac{\left(\Delta v^{8 / 3}-\Delta u^{8 / 3}\right)}{|\Delta v-\Delta u|}\right] .
$$

For the propagation of a single coherent mode $\psi_{k}(\vec{u})$, the average received 
intensity can be expressed as

$$
\begin{aligned}
\mu_{k} & =\left\langle\mathcal{I}_{k}\right\rangle=\iint_{\mathcal{A}^{2}} \psi_{k}^{*}\left(\vec{u}_{1}\right) \int w(\vec{v})\left\langle\mathcal{H}\left(\vec{u}_{1}, \vec{u}_{2}\right)\right\rangle \mathrm{d} \vec{v} \psi_{k}\left(\vec{u}_{2}\right) \mathrm{d} \vec{u}_{1} \mathrm{~d} \vec{u}_{2} \\
& =\left(\frac{1}{\lambda d}\right)^{2} \iint_{\mathcal{A}^{2}} \xi_{k}^{*}\left(\vec{u}_{1}\right) W\left(\frac{\vec{u}_{1}-\vec{u}_{2}}{\lambda d}\right) \exp \left[-\frac{1}{2} D_{\psi}(\Delta v=0, \Delta u)\right] \xi_{k}\left(\vec{u}_{2}\right) \mathrm{d} \vec{u} \mathrm{~d} \vec{u}_{2},
\end{aligned}
$$

where

$$
\xi_{k}(\vec{u})=\psi_{k}(\vec{u}) e^{j \frac{\pi}{\lambda d}\|\vec{u}\|^{2}} .
$$

\subsubsection{Second moment of the integrated intensity}

The second order moment of the received intensity from two transmitted coherent modes can be derived as

$$
\begin{aligned}
\left\langle\mathcal{I}_{k} \mathcal{I}_{l}\right\rangle= & \iiint \int_{\mathcal{A}^{4}} \psi_{k}^{*}\left(\vec{u}_{1}\right) \psi_{l}^{*}\left(\vec{u}_{3}\right) \psi_{k}\left(\vec{u}_{2}\right) \psi_{l}\left(\vec{u}_{4}\right) \iint_{\mathcal{R}^{2}} w\left(\vec{v}_{1}\right) h^{*}\left(\vec{v}_{1}, \vec{u}_{1}\right) h\left(\vec{v}_{1}, \vec{u}_{2}\right)(5.29) \\
& \times \Gamma_{4}\left(\vec{v}_{1}, \vec{v}_{2}, \vec{u}_{1}, \vec{u}_{2}, \vec{u}_{3}, \vec{u}_{4}\right) w\left(\vec{v}_{2}\right) h^{*}\left(\vec{v}_{2}, \vec{u}_{3}\right) h\left(\vec{v}_{2}, \vec{u}_{4}\right) \mathrm{d} \vec{v}_{1} \mathrm{~d} \vec{v}_{2} \mathrm{~d} \vec{u}_{1} \mathrm{~d} \vec{u}_{2} \mathrm{~d} \vec{u}_{3} \mathrm{~d} \vec{u}_{4}
\end{aligned}
$$

where $\Gamma_{4}\left(\vec{v}_{1}, \vec{v}_{2}, \vec{u}_{1}, \vec{u}_{2}, \vec{u}_{3}, \vec{u}_{4}\right)$ is the fourth moment of the turbulence part

$$
\begin{aligned}
& \Gamma_{4}\left(\vec{v}_{1}, \vec{v}_{2} ; \vec{u}_{1}, \vec{u}_{2}, \vec{u}_{3}, \vec{u}_{4}\right) \\
= & \left\langle\exp \left[\psi^{*}\left(\vec{v}_{1}, \vec{u}_{1}\right)+\psi\left(\vec{v}_{1}, \vec{u}_{2}\right)+\psi^{*}\left(\vec{v}_{2}, \vec{u}_{3}\right)+\psi\left(\vec{v}_{2}, \vec{u}_{4}\right)\right]\right\rangle \\
= & \exp \left(-\frac{1}{2} C\right) .
\end{aligned}
$$


The term $C$ in the above equation has been derived as [24]:

$$
\begin{aligned}
C= & D_{\psi}\left(\vec{v}_{1}, \vec{v}_{1}, \vec{u}_{1}, \vec{u}_{2}\right)+D_{\psi}\left(\vec{v}_{1}, \vec{v}_{2}, \vec{u}_{1}, \vec{u}_{4}\right)+D_{\psi}\left(\vec{v}_{1}, \vec{v}_{2}, \vec{u}_{2}, \vec{u}_{3}\right) \\
& +D_{\psi}\left(\vec{v}_{2}, \vec{v}_{2}, \vec{u}_{3}, \vec{u}_{4}\right)+D_{\psi}\left(\vec{v}_{1}, \vec{v}_{2}, \vec{u}_{2}, \vec{u}_{4}\right)+D_{\psi}\left(\vec{v}_{1}, \vec{v}_{2}, \vec{u}_{1}, \vec{u}_{3}\right) \\
& -4 B_{\chi}\left(\vec{v}_{1}, \vec{v}_{2}, \vec{u}_{2}, \vec{u}_{4}\right)-4 B_{\chi}\left(\vec{v}_{1}, \vec{v}_{2}, \vec{u}_{1}, \vec{u}_{3}\right) \\
& -j 2 D_{\chi \phi}\left(\vec{v}_{1}, \vec{v}_{2}, \vec{u}_{2}, \vec{u}_{4}\right)+j 2 D_{\chi \phi}\left(\vec{v}_{1}, \vec{v}_{2}, \vec{u}_{1}, \vec{u}_{3}\right),
\end{aligned}
$$

where

$$
\begin{aligned}
D_{\chi \phi}\left(\vec{v}_{1}, \vec{v}_{2}, \vec{u}_{1}, \vec{u}_{2}\right) & =\left\langle\left[\chi\left(\vec{v}_{1}, \vec{u}_{1}\right)-\chi\left(\vec{v}_{2}, \vec{u}_{2}\right)\right]\left[\phi\left(\vec{v}_{1}, \vec{u}_{1}\right)-\phi\left(\vec{v}_{2}, \vec{u}_{2}\right)\right]\right\rangle \\
B_{\chi}\left(\vec{v}_{1}, \vec{v}_{2}, \vec{u}_{1}, \vec{u}_{2}\right) & =\left\langle\chi\left(\vec{v}_{1}, \vec{u}_{1}\right) \chi\left(\vec{v}_{2}, \vec{u}_{2}\right)\right\rangle-\langle\chi\rangle^{2}
\end{aligned}
$$

In strong and moderately strong turbulence, it has been shown that the $B_{\chi}$ and $D_{\chi \phi}$ terms can be ignored [21]. So finally, we get

$$
\begin{aligned}
C \simeq & D_{\psi}\left(\vec{v}_{1}, \vec{v}_{1}, \vec{u}_{1}, \vec{u}_{2}\right)+D_{\psi}\left(\vec{v}_{1}, \vec{v}_{2}, \vec{u}_{1}, \vec{u}_{4}\right)+D_{\psi}\left(\vec{v}_{1}, \vec{v}_{2}, \vec{u}_{2}, \vec{u}_{3}\right) \\
& +D_{\psi}\left(\vec{v}_{2}, \vec{v}_{2}, \vec{u}_{3}, \vec{u}_{4}\right)+D_{\psi}\left(\vec{v}_{1}, \vec{v}_{2}, \vec{u}_{2}, \vec{u}_{4}\right)+D_{\psi}\left(\vec{v}_{1}, \vec{v}_{2}, \vec{u}_{1}, \vec{u}_{3}\right),(5
\end{aligned}
$$

where $D_{\psi}$ is the structure function in equation (5.26). It is clear that the second moment of the received intensity $\left\langle\mathcal{I}_{k} \mathcal{I}_{l}\right\rangle$ can be evaluated numerically from the above results. But the computation complexity exceeds the power of our workstations. 


\subsection{Selection of coherent modes}

The optimal coherent modes of $J\left(\vec{u}_{1}, \vec{u}_{2}\right)$ to minimize the scintillation index have been shown in [63] to be the fields maximizing the quadratic form in equation (5.12) as

$$
\hat{\Psi}=\arg \max _{\text {orthonormal } \Psi} \sum_{k} \sum_{l} \mu_{k}(\Psi) R_{k l}^{-1}(\Psi) \mu_{l}(\Psi) .
$$

For different turbulence cases, the optimal coherence modes $\Psi=\left\{\psi_{k}(\vec{u})\right\}$ and associated weights $\left\{\alpha_{k}\right\}$ vary with time. In practice, it is difficult to get these coherent modes for certain turbulence cases. Also, it is not reasonable to generate sets of orthogonal modes for different desired partial coherence. Fortunately, many laser modes generated by the laser resonator are beam like solutions of the paraxial wave equation. So it is natural to consider using these commonly used modes sets as the eigen modes set of $J\left(\vec{u}_{1}, \vec{u}_{2}\right)$.

There are several complete orthogonal solution families of the paraxial wave equation that are sometimes called the eigen modes of the PWE (Paraxial Wave Equation). These solution sets, such as the Hermite-Gaussian beams and the Laguerre-Gaussian beams introduced in Section 3.2, can be used as the coherent modes to generate partially coherent sources. 


\subsubsection{Scintillation reduction by using Hermite-Gaussian beams}

According to Section 3.2, the field amplitude of one dimensional HermiteGaussian beam at plane $z=0$ can be expressed as

$$
f_{m}(u)=\left(\frac{2}{\pi w_{0}^{2}}\right)^{1 / 4} \frac{1}{\sqrt{2^{m} m !}} H_{m}\left(\frac{\sqrt{2} u}{w_{0}}\right) \exp \left(-\frac{u^{2}}{w_{0}^{2}}\right),
$$

where $H_{m}(u)$ is the Hermite polynomial of order $m$, and $w_{0}$ is the spot size. The functions are normalized to represent a fixed amount of total beam power in all the modes

$$
\int_{\mathcal{A}}\left|f_{m}(u)\right|^{2} \mathrm{~d} u=1, \quad m=0,1,2, \cdots
$$

Plots of the amplitude of one dimensional Hermite-Gaussian beams $f_{m}(x)$ are shown in Figure 5.1. To focus the beams at the target plane $z=d$, a quadratic phase is included in the Hermite-Gaussian beam to form the coherent modes

$$
\psi_{m}(u)=f_{m}(u) \exp \left(-j \frac{\pi}{\lambda d}|u|^{2}\right)
$$

We set up a one dimensional propagation simulation with rectangular transmitter $\mathcal{A}$ and receiver region $\mathcal{R}$ (weighting function $w$ ) in random phase screen turbulence model to study the scintillation reduction by using partially coherent beams. The propagation parameters are shown in Table 5.1.

When a single normalized coherent mode $f_{m}(x)$ is transmitted to the target, the integrated intensity $\mathcal{I}_{m}$ through free space, the average integrated 

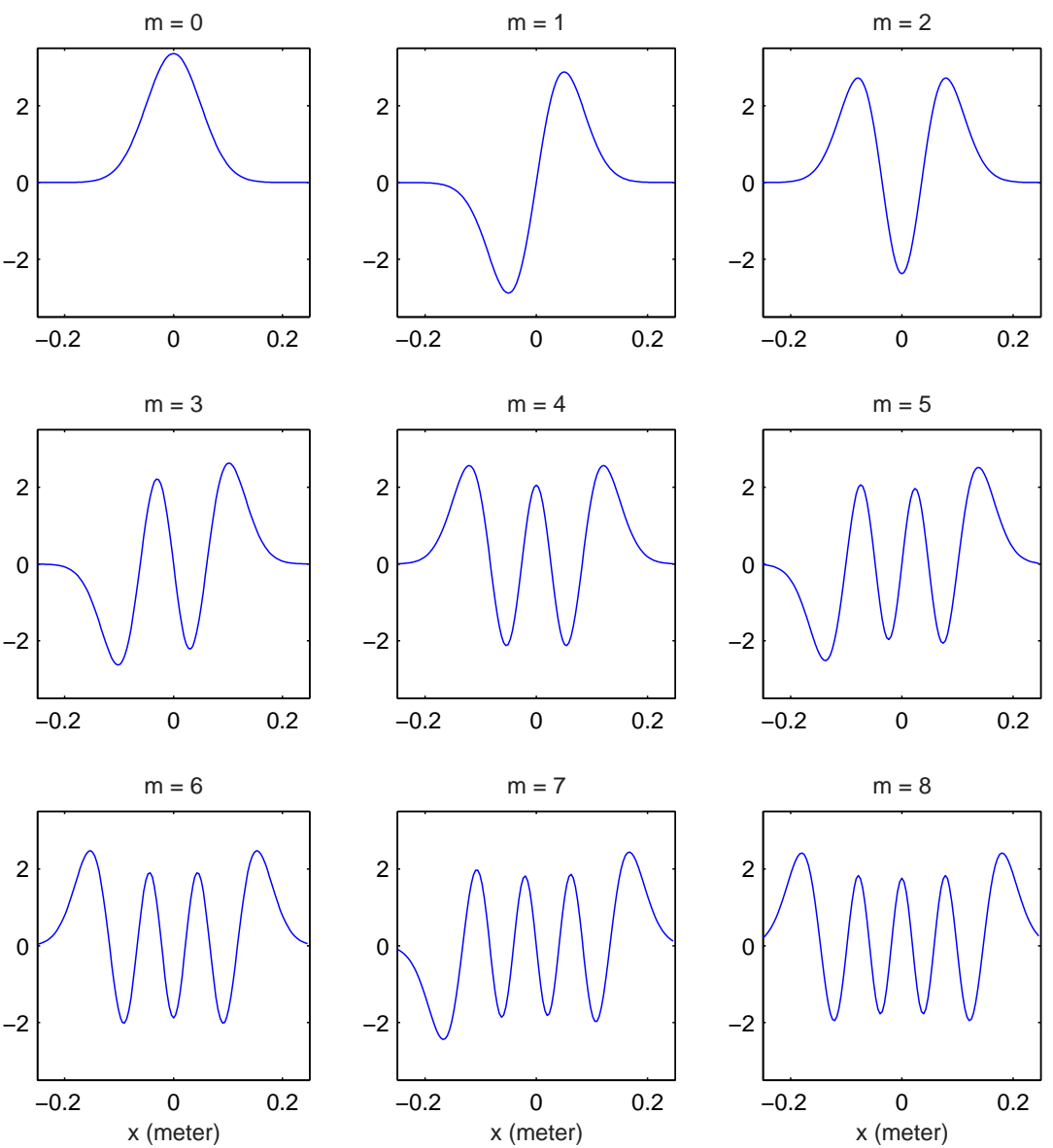

Figure 5.1: Amplitude of one dimensional Hermite-Gaussian beams $f_{m}(x)$.

\begin{tabular}{rccl}
\hline \hline Physical parameter & Symbol & \multicolumn{2}{c}{ Value } \\
\hline beam waist & $w_{0}$ & 0.05 & $\mathrm{~m}$ \\
wave length & $\lambda$ & 1.0 & $\mu \mathrm{m}$ \\
propagation distance & $d$ & 20 & $\mathrm{~km}$ \\
transmitter diameter & $D$ & 0.5 & $\mathrm{~m}$ \\
receiver radius & $r_{w}$ & 0.1 & $\mathrm{~m}$ \\
Fried parameter & $r_{0}$ & 10 & $\mathrm{~cm}$ \\
\hline
\end{tabular}

Table 5.1: Propagation parameters used in the one dimensional simulation. 


\begin{tabular}{c|c|c|c}
\hline \hline$m$ & $\mathcal{I}_{m}$ & $\mu_{m}$ & $S_{m}$ \\
\hline 0 & 0.9249 & 0.6419 & 0.1303 \\
1 & 0.6317 & 0.5513 & 0.0655 \\
2 & 0.3138 & 0.4792 & 0.0495 \\
3 & 0.2997 & 0.4222 & 0.0542 \\
4 & 0.3125 & 0.3771 & 0.0555 \\
5 & 0.2128 & 0.3411 & 0.0543 \\
6 & 0.2408 & 0.3120 & 0.0554 \\
7 & 0.2144 & 0.2878 & 0.0543 \\
8 & 0.1816 & 0.2667 & 0.0515 \\
9 & 0.2105 & 0.2463 & 0.0500 \\
\hline \hline
\end{tabular}

Table 5.2: Fractional integrated intensity $\xi_{m}$ in free space, fractional average integrated intensity $\mu_{m}$ through turbulence, and the scintillation index $S_{m}$ of the received fields by transmitting single Hermite-Gaussian modes $f_{m}(u)$.

intensity $\mu_{m}$ through turbulence and the scintillation index of the received fields have been shown in Table 5.2.

From the results in the previous section, we can derive the minimum scintillation index and corresponding optimal weighting $\alpha_{m}$ numerically when multiple Hermite-Gaussian modes are used to produce the optimal partially

\begin{tabular}{c|c|c|c|c|c|c|c|c}
\hline \hline$m$ & 0 & 1 & 2 & 3 & 4 & 5 & 6 & 7 \\
\hline 0 & 0.4657 & 0.3694 & 0.3065 & 0.2631 & 0.2325 & 0.2102 & 0.1931 & 0.1792 \\
1 & 0.3694 & 0.3239 & 0.2681 & 0.2300 & 0.2037 & 0.1837 & 0.1678 & 0.1549 \\
2 & 0.3065 & 0.2681 & 0.2410 & 0.2078 & 0.1812 & 0.1615 & 0.1469 & 0.1355 \\
3 & 0.2631 & 0.2300 & 0.2078 & 0.1880 & 0.1640 & 0.1454 & 0.1314 & 0.1202 \\
4 & 0.2325 & 0.2037 & 0.1812 & 0.1640 & 0.1501 & 0.1334 & 0.1193 & 0.1087 \\
5 & 0.2102 & 0.1837 & 0.1615 & 0.1454 & 0.1334 & 0.1227 & 0.1106 & 0.1001 \\
6 & 0.1931 & 0.1678 & 0.1469 & 0.1314 & 0.1193 & 0.1106 & 0.1027 & 0.0933 \\
7 & 0.1792 & 0.1549 & 0.1355 & 0.1202 & 0.1087 & 0.1001 & 0.0933 & 0.0873 \\
\hline \hline
\end{tabular}

Table 5.3: Mutual intensity $\left\langle\mathcal{I}_{m} \mathcal{I}_{n}\right\rangle$ by using Hermite Gaussian beams. 


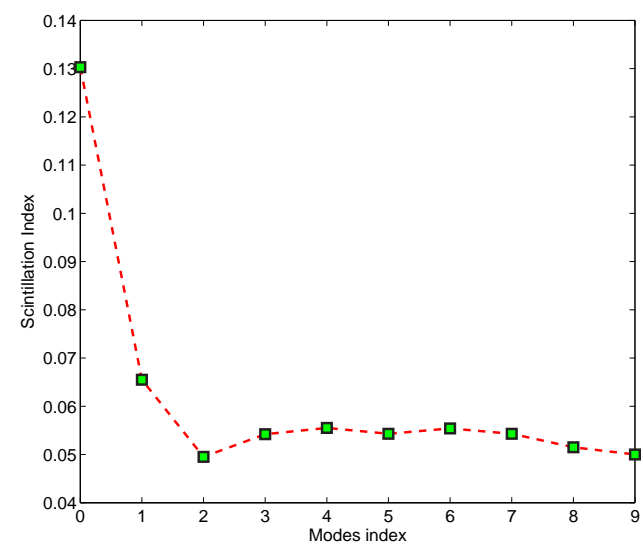

(a)

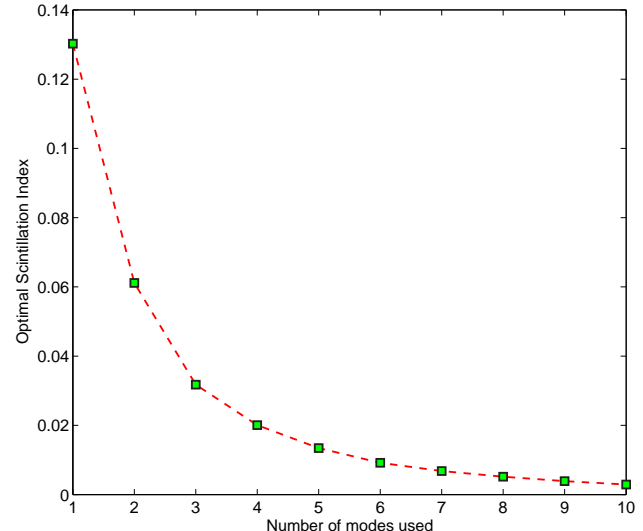

(b)

Figure 5.2: Scintillation index for transmitting Hermite-Gaussian modes: (a) Scintillation index for single modes. (b) Reduction of the optimal scintillation index when the number of Hermite-Gaussian modes increased.

coherence in the pupil plane. An evident reduction of the optimal scintillation index is shown in Figure 5.2 and the corresponding optimal parameters are shown in Table 5.4. At the same time, we note that the average integrated intensity by transmitting this optimal coherence drops down with the scintillation index reduction.

\subsubsection{Scintillation reduction by using Laguerre-Gaussian beams}

The Laguerre-Gaussian beam has been introduced in section 3.2. The corresponding one dimensional beam amplitude at transmitter plane $z=0$ can be expressed as

$$
f_{p, m}(r, \theta)=L_{p}^{m}\left(\frac{2 r^{2}}{w_{0}^{2}}\right)\left(\frac{\sqrt{2} r}{w_{0}}\right)^{m} \exp \left(-\frac{2 r^{2}}{w_{0}^{2}}\right),
$$




\begin{tabular}{c|c|c|c|c|c|c|c|c}
\hline \hline$N=$ & 1 & 2 & 3 & 4 & 5 & 6 & 7 & 8 \\
\hline$\alpha_{0}$ & 1.0000 & 0.1775 & 0.1472 & 0.1344 & 0.1305 & 0.1173 & 0.1032 & 0.0918 \\
$\alpha_{1}$ & & 0.8225 & 0.2185 & 0.2292 & 0.1544 & 0.1136 & 0.1040 & 0.1006 \\
$\alpha_{2}$ & & & 0.6343 & 0.0778 & 0.1442 & 0.1496 & 0.1309 & 0.0999 \\
$\alpha_{3}$ & & & & 0.5586 & 0.1132 & 0.1413 & 0.0959 & 0.1135 \\
$\alpha_{4}$ & & & & & 0.4577 & 0.0259 & 0.1665 & 0.1176 \\
$\alpha_{5}$ & & & & & & 0.4522 & 0.0000 & 0.0913 \\
$\alpha_{6}$ & & & & & & & 0.3995 & 0.0548 \\
$\alpha_{7}$ & & & & & & & & 0.3305 \\
\hline$\mu_{\mathcal{I}}$ & 0.6419 & 0.5674 & 0.5189 & 0.4858 & 0.4584 & 0.4333 & 0.4142 & 0.3979 \\
$S_{\mathcal{I}}$ & 0.1303 & 0.0611 & 0.0317 & 0.0201 & 0.0134 & 0.0092 & 0.0068 & 0.0052 \\
\hline \hline
\end{tabular}

Table 5.4: Optimal weighting $\alpha_{m}$, scintillation index $S_{I}$, and the fractional average integrated intensity $\mu$ when the first $N$ Hermite-Gaussian modes $f_{m}(x)$ are used to generate the optimal pupil plane partially coherence.

where $L_{p}^{m}$ is the generalized Laguerre polynomials. Plots of the normalized one dimensional Laguerre-Gaussian beam amplitude with $m=0$ have been shown in Figure 5.3.

We setup the same one dimensional propagation situation as which is used in the previous section with parameters in Table 5.1. When a single normalized coherent mode $f_{p, 0}(x)$ propagated to the target, the integrated intensity $\xi_{p}$ through free space, the average integrated intensity $\mu_{p}$ through turbulence and the scintillation index $S_{\mathcal{I}_{p}}$ of the received fields are shown in Table 5.5.

An evident reduction of the optimal scintillation index has been shown in Figure 5.4 and the corresponding optimal parameters are shown in Table 5.6. As in the case using Hermite-Gaussian modes, we noticed that the average integrated intensity drops down with the scintillation index reduction. 

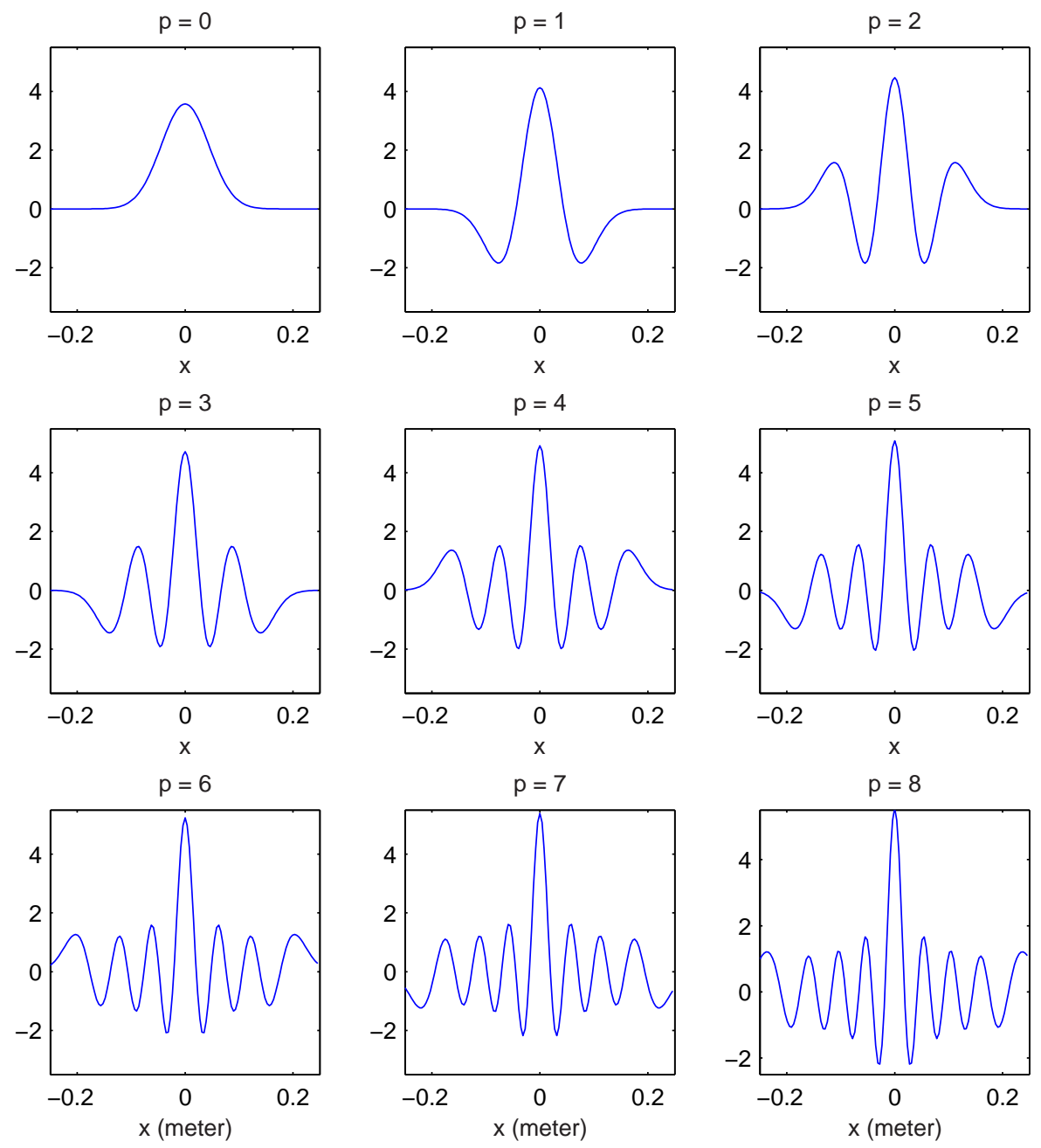

Figure 5.3: One dimensional Laguerre-Gaussian beams $f_{p, m}(x)$ with $m=0$. 


\begin{tabular}{c|c|c|c}
\hline \hline$p$ & $\xi_{p}$ & $\mu_{p}$ & $S_{p}$ \\
\hline 0 & 0.8981 & 0.6296 & 0.1285 \\
1 & 0.3743 & 0.4291 & 0.0350 \\
2 & 0.2810 & 0.3062 & 0.0745 \\
3 & 0.1343 & 0.2353 & 0.0755 \\
4 & 0.1564 & 0.1920 & 0.0574 \\
5 & 0.1601 & 0.1633 & 0.0427 \\
6 & 0.1157 & 0.1416 & 0.0376 \\
7 & 0.1129 & 0.1210 & 0.0375 \\
8 & 0.1030 & 0.0992 & 0.0387 \\
9 & 0.1007 & 0.0832 & 0.0358 \\
\hline \hline
\end{tabular}

Table 5.5: Fractional integrated intensity $\xi_{p}$ in free space, fractional average integrated intensity $\mu_{p}$ through turbulence, and the scintillation index $S_{p}$ of the received fields by transmitting single Laguerre-Gaussian modes $f_{p, 0}(x)$.

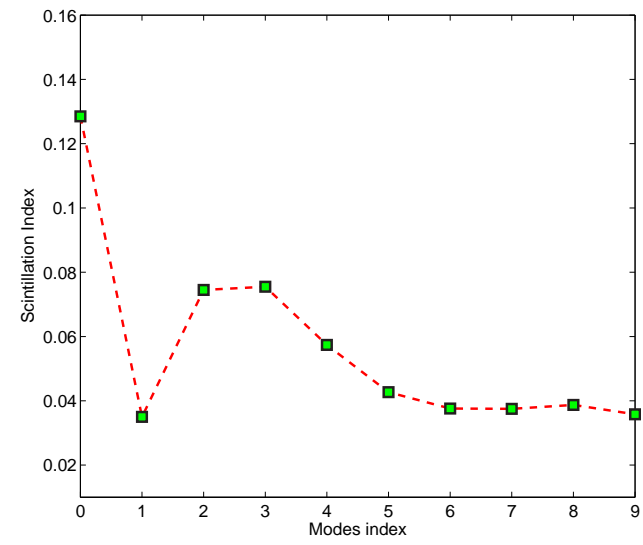

(a)

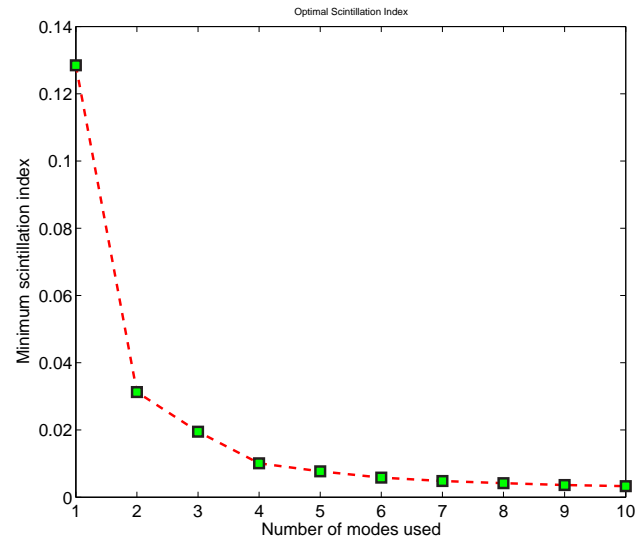

(b)

Figure 5.4: Scintillation index by transmitting Laguerre-Gaussian modes: (a) Scintillation index by transmitting single modes. (b) Reduction of the optimal scintillation index when the number of Laguerre-Gaussian modes increased 


\begin{tabular}{c|c|c|c|c|c|c|c|c}
\hline \hline$N$ & 1 & 2 & 3 & 4 & 5 & 6 & 7 & 8 \\
\hline$\alpha_{0}$ & 1.0000 & 0.1177 & 0.2403 & 0.2019 & 0.1579 & 0.1116 & 0.0935 & 0.0840 \\
$\alpha_{1}$ & & 0.8823 & 0.0718 & 0.0000 & 0.0367 & 0.0904 & 0.0835 & 0.0732 \\
$\alpha_{2}$ & & & 0.6879 & 0.3174 & 0.1838 & 0.0924 & 0.0910 & 0.0822 \\
$\alpha_{3}$ & & & & 0.4807 & 0.2203 & 0.1473 & 0.0577 & 0.0727 \\
$\alpha_{4}$ & & & & & 0.4013 & 0.0555 & 0.1783 & 0.0844 \\
$\alpha_{5}$ & & & & & & 0.5029 & 0.0000 & 0.0623 \\
$\alpha_{6}$ & & & & & & & 0.4960 & 0.2121 \\
$\alpha_{7}$ & & & & & & & & 0.3291 \\
\hline$\mu_{\mathcal{I}}$ & 0.6296 & 0.4527 & 0.3927 & 0.3374 & 0.3003 & 0.2648 & 0.2406 & 0.2228 \\
$S_{\mathcal{I}}$ & 0.1285 & 0.0313 & 0.0195 & 0.0103 & 0.0077 & 0.0058 & 0.0048 & 0.0042 \\
\hline \hline
\end{tabular}

Table 5.6: Optimal weighting $\alpha_{p}$, scintillation index $S_{\mathcal{I}}$, and the fractional average integrated intensity $\mu$ when the first $N$ Laguerre-Gaussian modes $f_{p, 0}(u)$ are used to generate the optimal pupil plane partially coherence.

The minimum scintillation index $S_{\mathcal{I}}$ and corresponding optimal weighting $\alpha_{p}$ are computed numerically when multiple Laguerre-Gaussian modes are used to produce the optimal partially coherence in the pupil plane.

\subsection{Monte-Carlo Simulation}

Because the multiple integral expressions in equation (5.20) and equation (5.29) can not be solved in closed form, analytical calculation of higher order statistics of the integrated intensity becomes very difficult. In our case, the numerical computation of the second moment of the integrated intensity is beyond the capacity of today's high speed computers, especially for two dimensional propagation cases. So we turn to Monte-Carlo simulation, which is widely used in the problem of optical beam propagation through turbulence.

In this simulation study, we generate random realizations of the phase 
screen in the pupil plane according to the structure function $D_{\phi}\left(\vec{u}_{1}, \vec{u}_{2}\right)$. By calculating the integrated intensity of different input beams for each realization of the pupil phase and accumulating the results, statistical properties (first and second moments) of the received intensity can be obtained.

To measure the accuracy of the Monte-Carlo simulation, we define a criterion, the mean square error $\epsilon^{2}$, as follows

$$
\epsilon^{2}=\frac{\sum\left(\frac{V_{m}-V_{t}}{V_{t}}\right)^{2}}{(M \times N)},
$$

where $V_{m}$ is the performance estimated from Monte-Carlo simulation and $V_{t}$ is the theoretical calculation result. The summation is taken over all elements in the matrices $V_{M \times N}$ and the error is measured per array elements by dividing $(M \times N)$, the number of elements in the array $V$.

We set up the propagation simulation by taking the same physical parameters that we used in Table 5.1, so that the Monte-Carlo simulation results can be compared with the theoretical calculation results that we obtained in the previous section.

\subsubsection{Phase screen generation}

There are many approaches that can be used to generate the random phase screens with proper statistical properties. A variety of phase screen generators have been introduced in the literature [58, 15, 29]. One simple approach based on linear combination of orthonormal basis functions gives good performance in terms of statistics matching. We use this approach in our simulation 


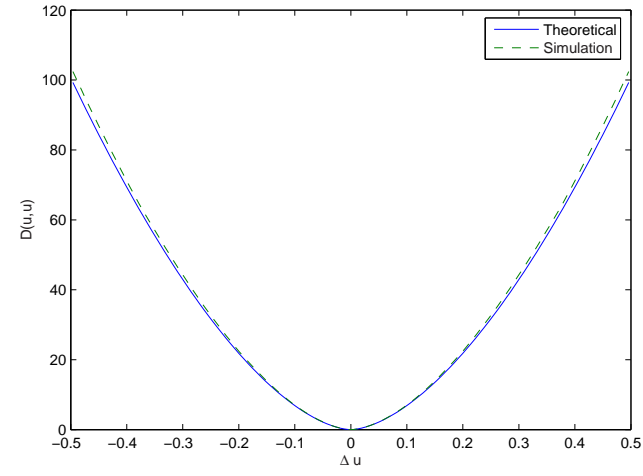

(a)

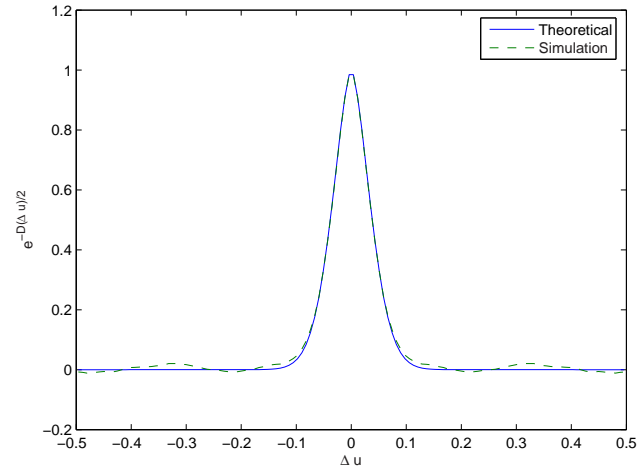

(b)

Figure 5.5: Theoretical and Monte-Carlo simulation of the phase structure function $D_{\phi}(\Delta u)$ and $\left\langle\exp \left[\phi\left(u_{1}\right)-\phi\left(u_{2}\right)\right]\right\rangle$ of the random phase screen

to generate random phase screens with the structure function expressed as

$$
D_{\phi}\left(u_{1}, u_{2}\right)=6.88\left(\frac{\left|u_{1}-u_{2}\right|}{r_{0}}\right)^{5 / 3}
$$

where $r_{0}$ is the Fried parameter. More details about this approach can be found in Section 3.7 of reference [58].

It is straight forward to see that the accuracy of the final simulation results depend on the accuracy of the random phase screens generated. The structure function $D_{\phi}\left(u_{1}, u_{2}\right)$ of the random phase and $\left\langle\exp \left[\phi\left(u_{1}\right)-\phi\left(u_{2}\right)\right]\right\rangle$ are taken as performance metrics and the mean square error can be calculated as in equation (5.38). Taking the computation across a data set of 1,000 random draws, the theoretical and Monte-Carlo simulation results are shown in Figure 5.5 and the square errors are shown in Table 5.7. 


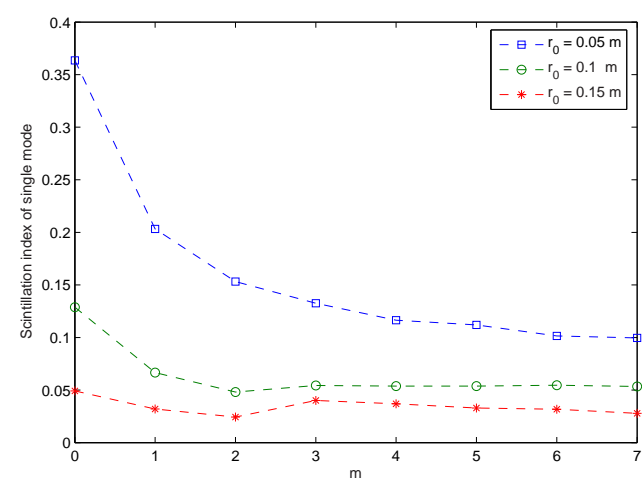

(a)

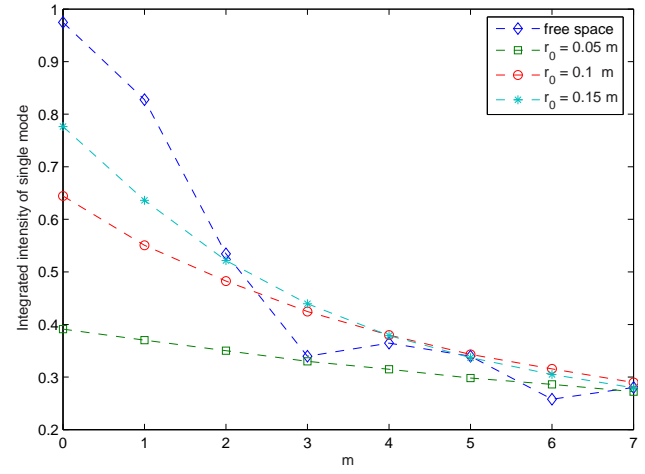

(b)

Figure 5.6: Monte-Carlo simulation by transmitting single one dimensional Hermite-Gaussian modes $f_{m}(x)$ : (a) Scintillation index, (b) average integrated intensity.

\subsubsection{Pupil plan phase screen simulation}

Monte-Carlo simulation for the pupil plane phase screen model is taken first since the theoretical calculation results in one dimensional propagation are available which can be used for comparison with the simulation results.

\section{One-dimensional Monte-Carlo simulation}

By transmitting a single one dimensional Hermite-Gaussian mode $f_{m}(u)$ through the simulated random phase screens, the average integrated intensity and the scintillation index at the receiver are shown in Figure 5.6.

When multiple Hermite-Gaussian modes are used to synthesize the optimal pupil plane coherence and propagate through the turbulence, the average integrated intensity and the scintillation index are shown in Figure 5.7. Evident reduction of the scintillation index can be seen. 


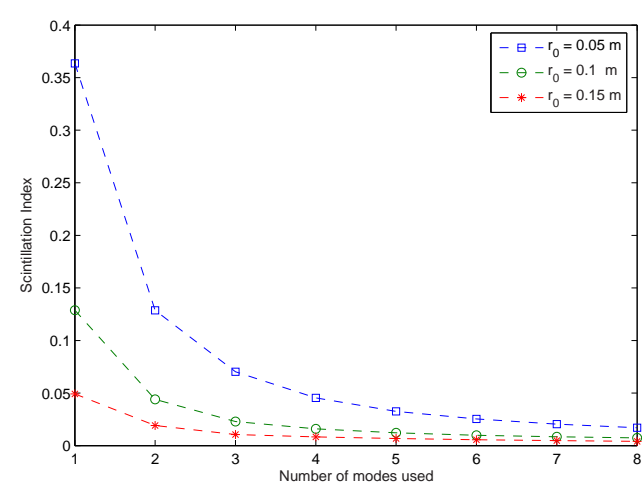

(a)

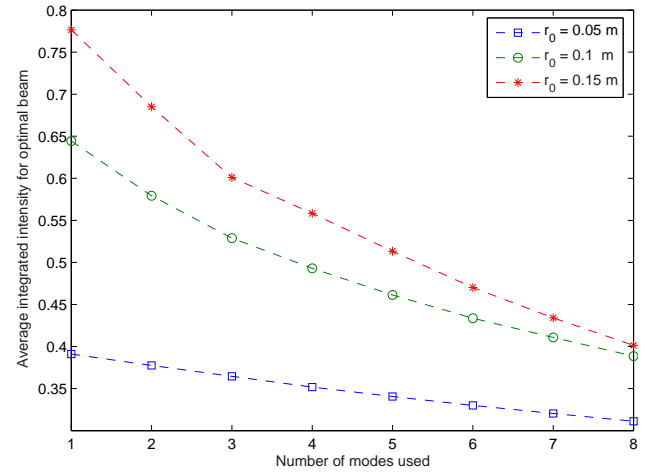

(b)

Figure 5.7: Monte-Carlo simulation by transmitting the optimal coherence composed of multiple one dimensional Hermite-Gaussian modes $f_{m}(x)$ : (a) Scintillation index, (b) average integrated intensity.

\begin{tabular}{c|c|c|c|c}
\hline \hline & $D_{\phi}$ & $\mu_{m}$ & $S_{m}$ & $\left\langle\mathcal{I}_{m} \mathcal{I}_{n}\right\rangle$ \\
\hline$\epsilon^{2}$ & $1.4835 \times 10^{-4}$ & $4.4090 \times 10^{-5}$ & $2.1388 \times 10^{-4}$ & $7.8846 \times 10^{-4}$ \\
\hline \hline
\end{tabular}

Table 5.7: Mean square error $\epsilon^{2}$ of the Monte-Carlo simulation for different performance values: $\mu_{m}, S_{m}$ and $\left\langle\mathcal{I}_{m} \mathcal{I}_{n}\right\rangle$.

Compare the above simulation results with the theoretical calculations in Section 5.4.1, the mean square error of the fractional average integrated intensity $\mu_{m}$, of the scintillation index of single transmitted mode $S_{m}$, of the average mutual received intensity array are shown in Table 5.7.

The above results show that Monte-Carlo simulation matches the theoretical calculations very well for the first and second moments of the integrated intensities.

The simulation results, achieved by transmitting one dimensional LaguerreGaussian beams and the performance of the optimal coherence composed of multiple Laguerre-Gaussian modes, are shown in Figure 5.8 and Figure 5.9 


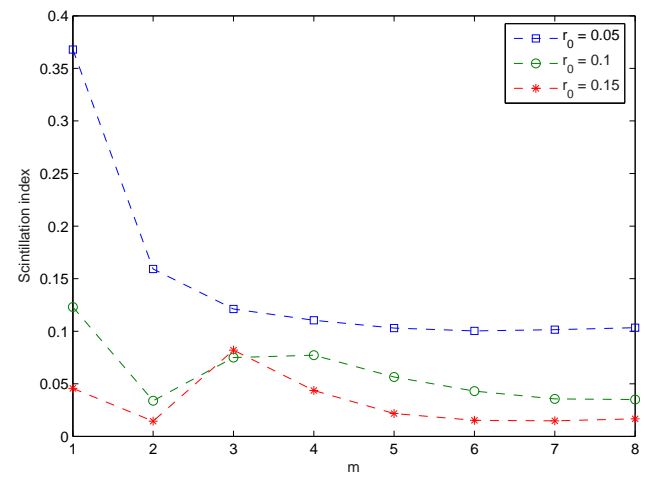

(a)

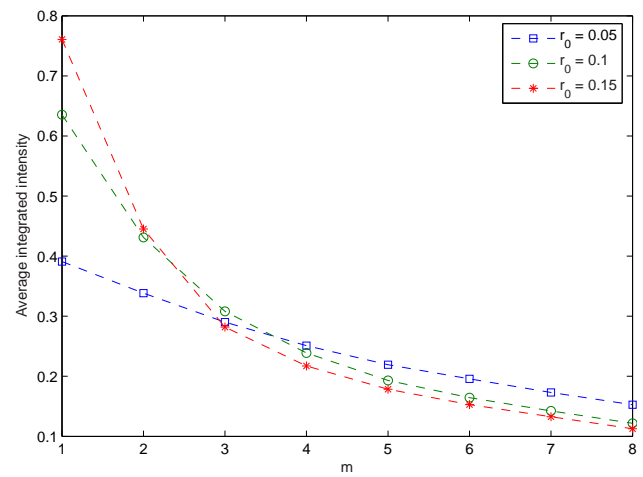

(b)

Figure 5.8: Monte-Carlo simulation by transmitting single one dimensional Laguerre-Gaussian modes $f_{m, 0}(x)$ : (a) Scintillation index, (b) average integrated intensity.

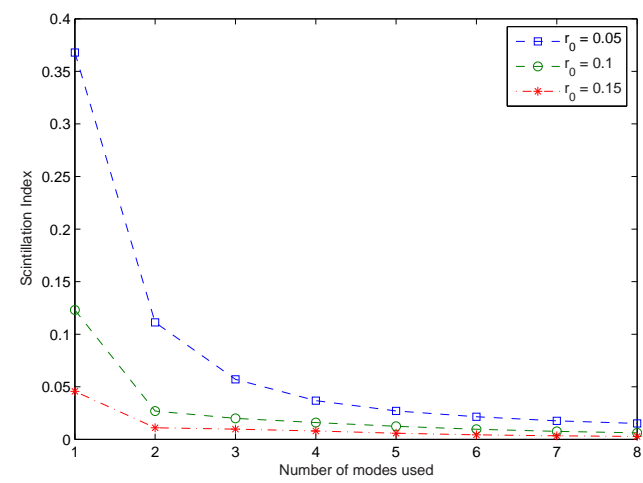

(a)

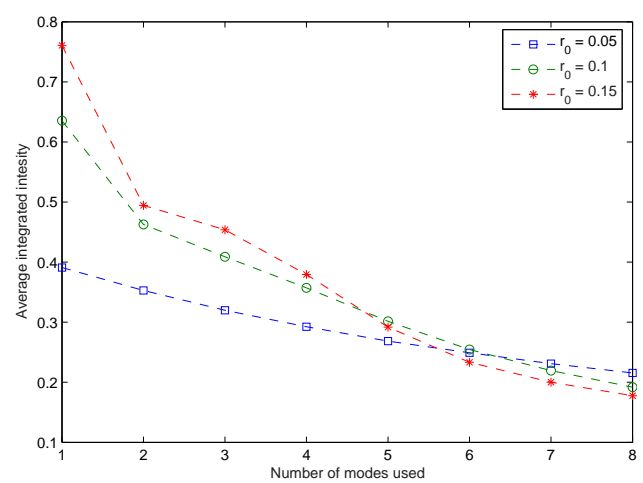

(b)

Figure 5.9: Monte-Carlo simulation by transmitting the optimal coherence composed of multiple one dimensional Laguerre-Gaussian modes $f_{m, 0}(x)$ : (a) Scintillation index, (b) average integrated intensity. 


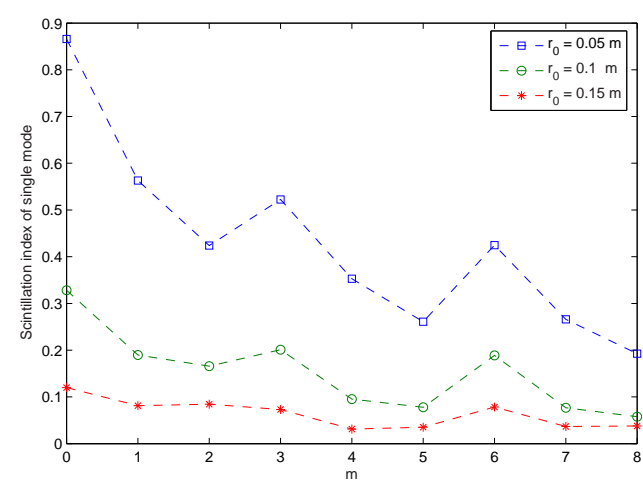

(a)

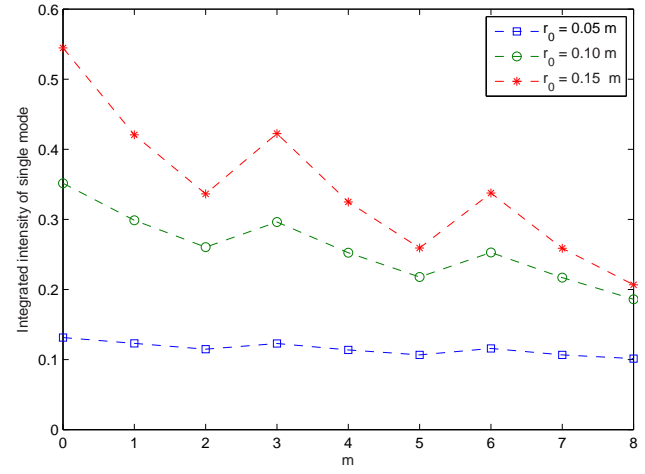

(b)

Figure 5.10: Monte-Carlo simulated performance of transmitting single two dimensional Hermite-Gaussian beam: (a) Scintillation index, (b) average integrated intensity.

\section{Two-dimensional Monte-Carlo simulation}

Because the Monte-Carlo simulation in one dimensional case showed good performance in terms of matching theoretical calculations well, it is natural to extend it to two dimensional cases. Without changing the physical propagation parameters, the first and second moments of the integrated intensity, the minimum scintillation index, and the associated optimal weighting are shown in the following figures and table. As in the one dimensional case, evident reduction of the scintillation index can be seen when the optimal pupil plane coherence generated by multiple beams are propagated to the target. But the average integrated intensity drops down also with the increasing of the number of coherent modes used.

Monte-Carlo simulation by transmitting two dimensional Hermite-Gaussian beams and the optimal pupil plane coherence composed of Hermite-Gaussian 


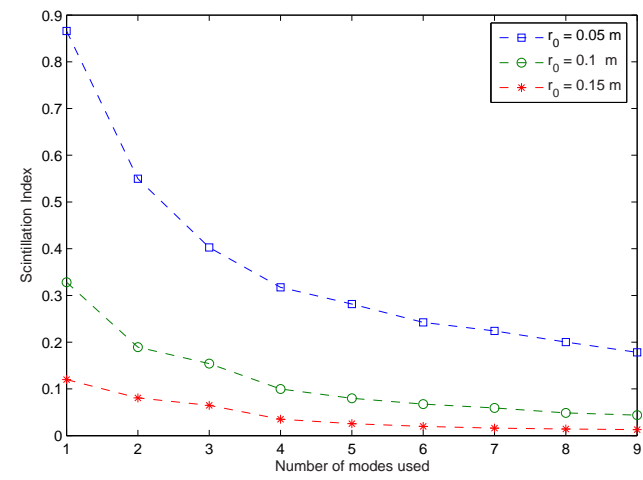

(a)

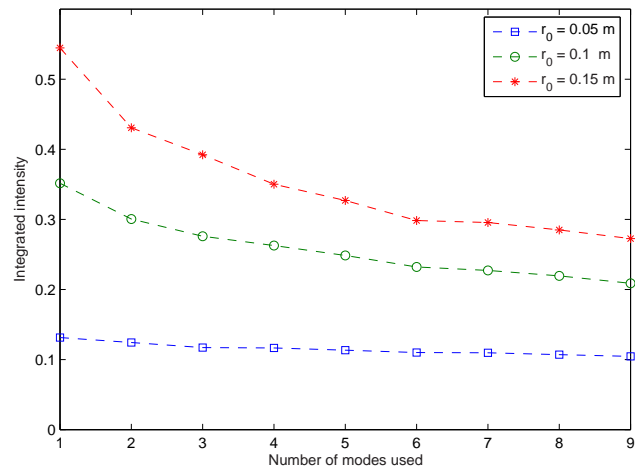

(b)

Figure 5.11: Monte-Carlo simulated performance of transmitting the optimal pupil plane coherence composed of multiple two dimensional HermiteGaussian beams: (a) Scintillation index, (b) average integrated intensity.

modes are shown in Figure 5.10 and Figure 5.11. Simulation results of transmitting two dimensional Laguerre-Gaussian beams and the optimal pupil plane coherence composed of Laguerre-Gaussian modes are shown in Figure 5.12 and Figure 5.13.

\subsubsection{Multiple phase screen simulation}

We set up the Monte-Carlo simulation by using multiple phase screens to simulate the turbulent propagation path. The von Karman spectrum is used and four phase screens are set equally distanced from the transmitter pupil to the target. In this simulation, the FFT method are used to generate multiple phase screens following von Karman turbulence spectrum. Four random phase screens are put in the propagation path from pupil to receiver with equal distance. Physical parameters used in the simulation are shown 


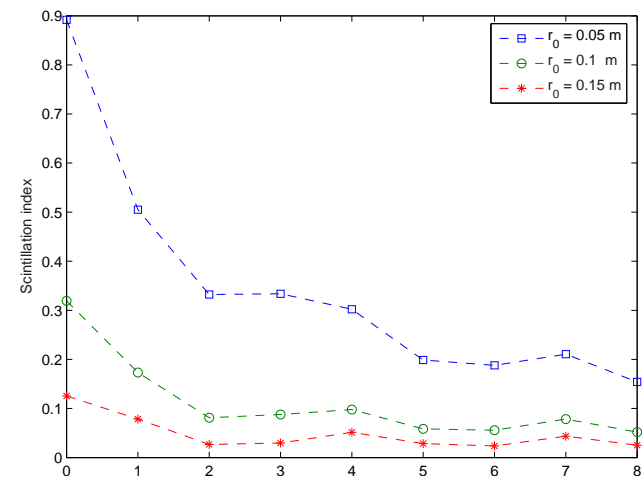

(a)

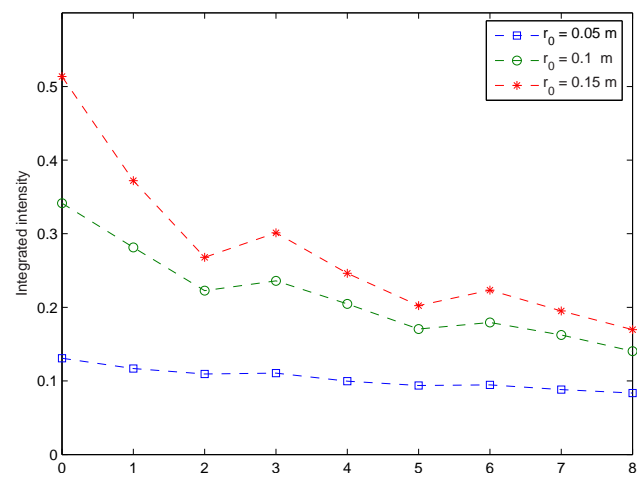

(b)

Figure 5.12: Monte-Carlo simulated performance of transmitting single two dimensional Laguerre-Gaussian beams: (a) scintillation index, (b) average integrated intensity.

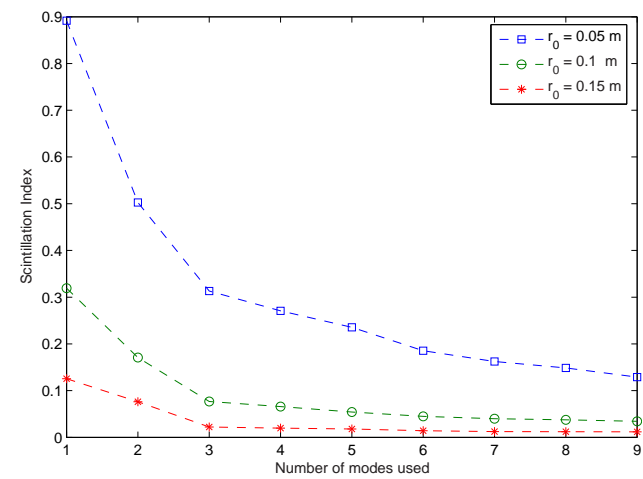

(a)

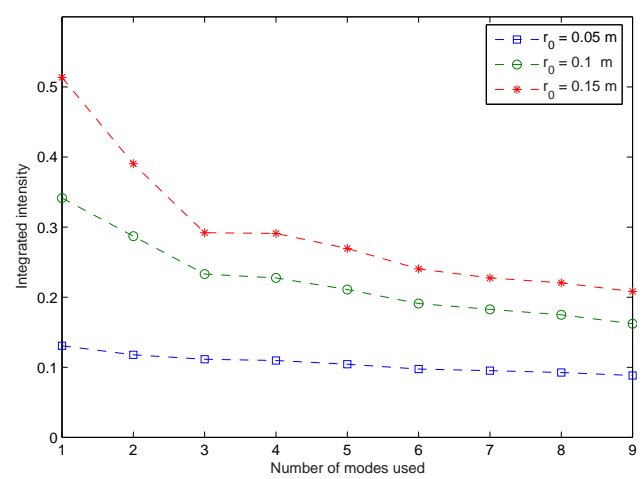

(b)

Figure 5.13: Monte-Carlo simulated performance of the optimal coherence composed of multiple two dimensional Laguerre-Gaussian modes: (a) scintillation index, (b) associated average integrated intensity. 


\begin{tabular}{lcrl}
\hline \hline Physical parameter & Symbol & Value(s) \\
\hline transmitter diameter & $D$ & 0.5 & $\mathrm{~m}$ \\
propagation distance & $d$ & 20 & $\mathrm{~km}$ \\
wave length & $\lambda$ & 1.0 & $\mu \mathrm{m}$ \\
beam waist & $w_{0}$ & 5 & $\mathrm{~cm}$ \\
receiver radius & $r$ & 8 & $\mathrm{~cm}$ \\
inner scale & $l_{0}$ & $10 \mathrm{~m}$ \\
outer scale & $L_{0}$ & $\mathrm{~m}$ \\
structure constant & $C_{n}^{2}$ & $2,5,9 \times 10^{-16}$ & $\mathrm{~m}^{-2 / 3}$ \\
\hline \hline
\end{tabular}

Table 5.8: Parameters used in the Monte-Carlo simulation of laser beams propagate through multiple random phase screens.

in Table 5.8.

By using two dimensional Hermite-Gaussian beams, the scintillation index and average integrated intensity by transmitting single coherent modes are shown in Figure 5.14. Corresponding performances by transmitting the optimal partial coherence are shown in Figure 5.15. It is easy to see that we get similar results as what we achieved in the pupil plane phase screen model. Monte-Carlo simulation results by using two dimensional Laguerre-Gaussian beams set are shown in Figure 5.16 and Figure 5.17.

\subsection{Aperture Averaging of Scintillation}

It is noticed that when the receiver aperture has a finite size $\mathcal{R}$ rather than a point receiver, the intensity fluctuations $S_{\mathcal{I}}(\mathcal{R})$ drop because of the averaging process over the aperture. This phenomenon is called aperture averaging, and has been studied by many researchers [73, 26, 79, 57]. Aperture averaging effect (also called aperture smoothing) can help us to understand the well 


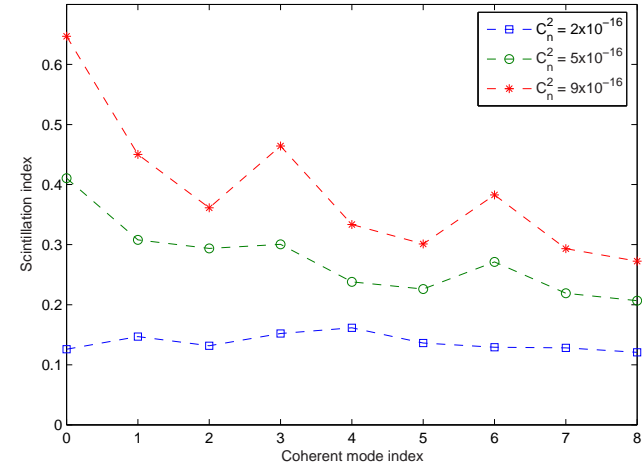

(a)

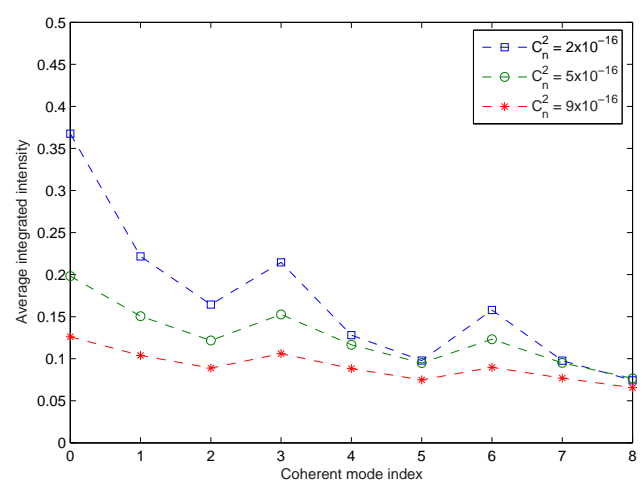

(b)

Figure 5.14: Monte-Carlo simulated performance of transmitting single two dimensional Hermite-Gaussian beams through multiple phase screens: (a) scintillation index, (b) average integrated intensity.

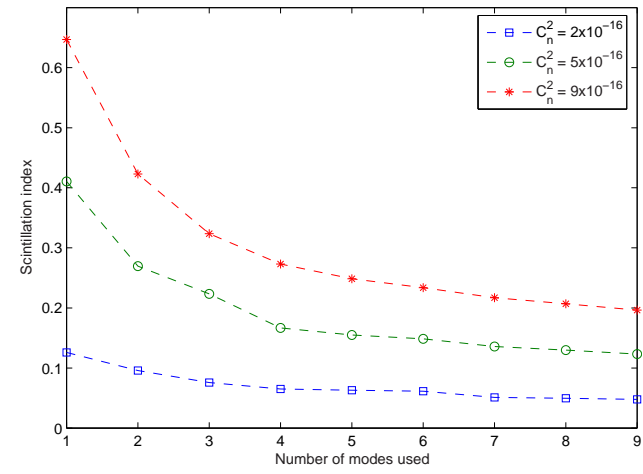

(a)

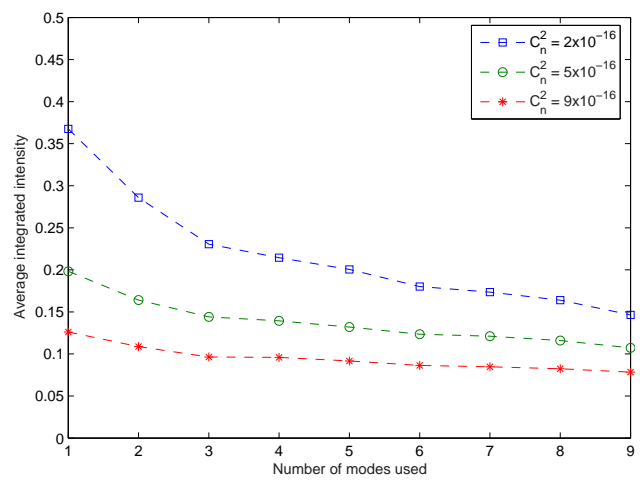

(b)

Figure 5.15: Monte-Carlo simulated performance of transmitting optimal partial coherence synthesized by two dimensional Hermite-Gaussian beams through multiple phase screens: (a) scintillation index, (b) average integrated intensity. 


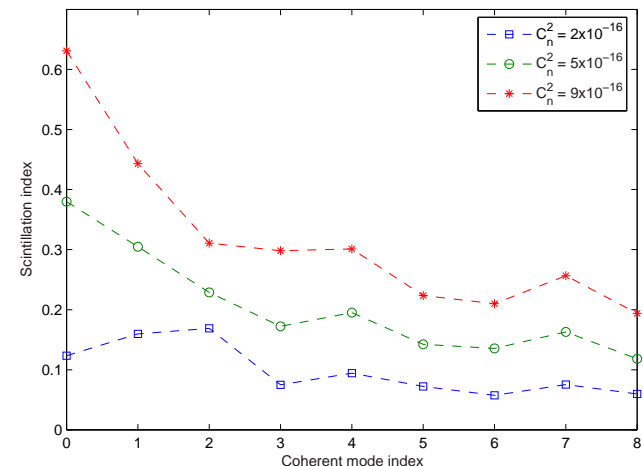

(a)

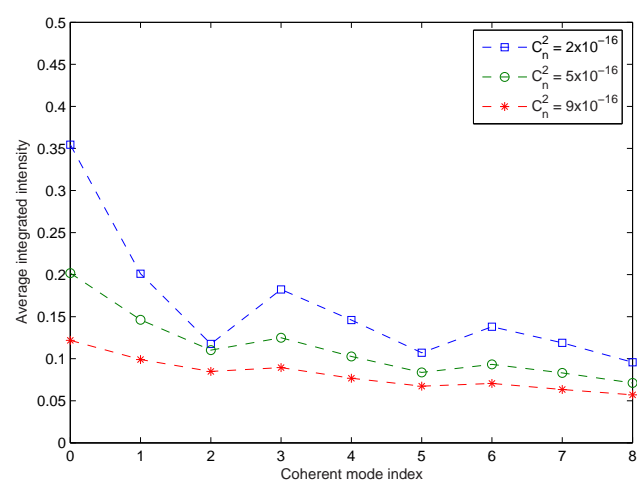

(b)

Figure 5.16: Monte-Carlo simulated performance of transmitting single two dimensional Laguerre-Gaussian beams through multiple phase screens: (a) scintillation index, (b) average integrated intensity.

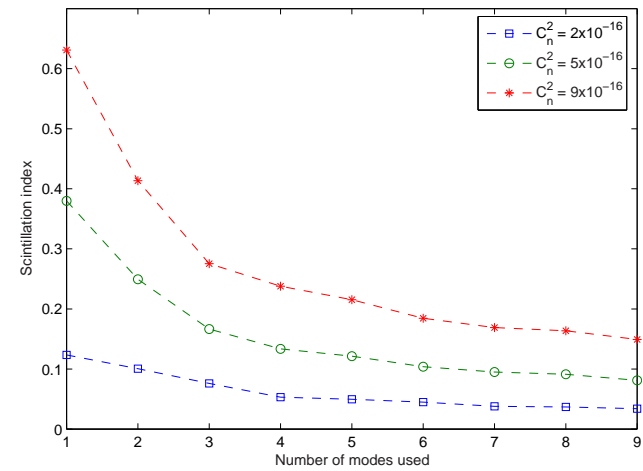

(a)

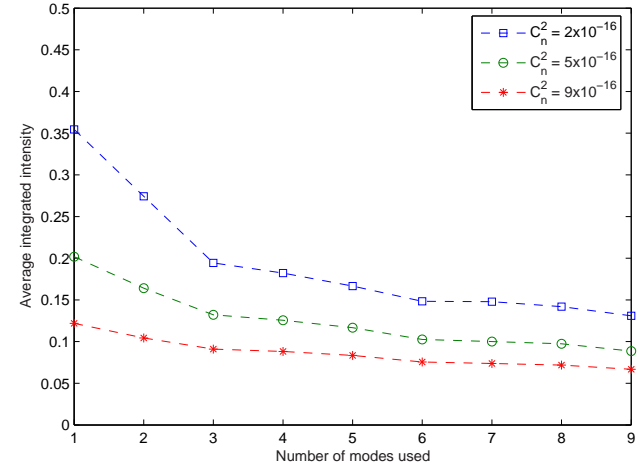

(b)

Figure 5.17: Monte-Carlo simulated performance of transmitting optimal partial coherence synthesized by two dimensional Laguerre-Gaussian beams through multiple phase screens: (a) scintillation index, (b) average integrated intensity. 
known fact that planets scintillate less than stars when viewed at the same zenith angle [80].

The ratio

$$
A(\mathcal{R})=\frac{S_{\mathcal{I}}(\mathcal{R})}{S_{\mathcal{I}}(0)}
$$

is defined as the aperture averaging factor [73], which is used to measure the intensity fluctuation decrease as the receiver aperture size increases. In this section, we will show how the aperture averaging process affects the scintillation index reduction when the optimal pupil plane coherence are used in this section.

A simulation of one dimensional propagation in the random phase screen model was set up with the same physical parameters as were used in the previous two sections. When single Hermite-Gaussian modes are transmitted, the scintillation index changes vs. the receiver size that has been shown in Figure 5.18 (a). It can be seen that there are evident aperture averaging effects for every coherent mode when the receiver radius increased.

When multiple Hermite-Gaussian modes are used to synthesize the optimal pupil plane coherence, the changes of scintillation index vs. the receiver size has been shown in Figure 5.18 (b). It can be seen that the aperture averaging effect is evident when only one or two Hermite modes are used to synthesize the optimal coherence. But when the number of coherent modes is increased, the scintillation index does not change much with the aperture size, which means there is not much aperture averaging effect. It should be noticed that for certain aperture sizes, the scintillation index values in the 


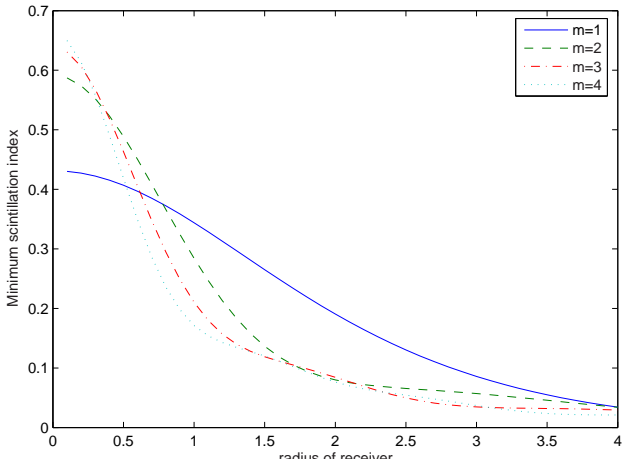

(a)

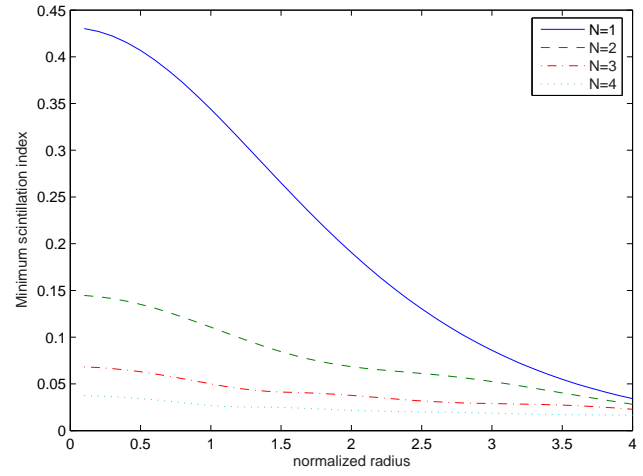

(b)

Figure 5.18: Aperture averaging effect in the pupil plane phase screen model: (a) Scintillation index vs. the normalized receiver radius for single Hermite Gaussian modes $f_{m}(x)$. (b) Optimal scintillation index vs. the normalized receiver radius for optimal beams synthesized by $N$ Hermite-Gaussian modes.

figure are the minimum one since the optimal pupil plane coherence are used. So we can conclude that by using the optimal pupil plane coherence, increasing receiver aperture size will not help to cancel optical field scintillation. 


\section{Chapter 6}

\section{Summary}

\subsection{Conclusions}

In this work, we investigated the optimal pupil plane field for two different performance metrics to compensate for the turbulence effects on laser beam propagation through turbulence.

To compensate for laser beam spreading, the optimal beam to maximize the averaged integrated intensity has been investigated and shown to be fully coherent. In this study,

1. We have derived the averaged kernel $\left\langle\mathcal{H}\left(u, u^{\prime}\right)\right\rangle$ over turbulence for two different turbulence models: the turbulence layer model and the extended Huygens-Fresnel principle model. A similarity relationship between the two models has been shown.

2. An equivalence between the average integrated intensity in turbulence propagation and the integrated intensity in free space propagation with a new weighting function has been established. 
3. We have shown that the optimal coherent beam to maximize the average integrated intensity $\langle\mathcal{I}\rangle$ does not provide evident improvement. Mathematical analysis demonstrates that the eigenvalues of $\left\langle\mathcal{H}\left(u, u^{\prime}\right)\right\rangle$ distributed much more evenly because of the turbulence induced changes. There is not one beam that can perform much better than others. Short-term compensation is thus suggested.

The optimal pupil plane coherence to reduce the beam scintillation has been shown to be partially coherent in general cases. In this study,

1. We have derived the first and second moments of the received intensity by transmitting coherent modes in two turbulence models: the turbulence layer model and the extended Huygens-Fresnel principle model.

2. We have used the Hermite-Gaussian and Laguerre-Gaussian beams as the coherent beam set to synthesize the optimal partially coherent source. We derived the optimal weights $\alpha_{k}$ for coherent modes $\psi_{k}(u)$ of the optimal pupil plane mutual intensity $J\left(u, u^{\prime}\right)$ and have shown evident reduction of the scintillation index when optimal partially coherent sources are used. We also showed the trade-off effect, decreasing of the average integrated intensity.

3. We have shown the aperture averaging effects of the optimal beams. Comparison with the coherent beam showed the decreasing of aperture averaging when optimal partially coherent beams were used. We 
showed that increasing the receiver aperture size will not help when optimal pupil plane coherence are used.

\subsection{Future work}

It has been shown that partially coherent sources can effectively reduce the laser beam scintillation. But an important issue in practice is that a scheme to generate the partially coherent beams with desired optimal coherence is still not available.

In multi-mode laser cases, the lack of phase correlations between the modes can be used to generate partially coherent fields. Another possibility is to overlap multiple beams with independent phases. Recently, a new scheme [76] is proposed based on random phase modulation of a coherent beam source. In this method, the coherent beam passes through a phase diffusor with a limited number of spatial correlation zones. Along with the inherent time averaging of the photo-detection processes, a beam with partially coherent properties can be produced. In another approach [78], the non-linear effect in a single mode fiber is used to reduce the temporal coherence of the transmitted beam, and its high spatial coherence is then reduced.

Although multiple schemes have been proposed to generate partially coherent beams, there is one important unsolved problem with the existing scheme: it is difficult to control the generated coherence. To achieve the desired pupil plane partial coherence is still a difficult problem. We will investigate this problem in the future. 


\section{Appendix A}

\section{Glossary of Symbols}

$\begin{array}{ll}\mathcal{A} & \text { Transmitter pupil } \\ a(x) & \text { Aperture function } \\ c & \text { Speed of light } \\ C_{n}^{2} & \text { Structure constant of refractive index } \\ D & \text { Transmitter aperture diameter } \\ D_{f}(x) & \text { Structure function of fluctuation } f \\ d & \text { Propagation distance } \\ f(u) & \text { Optical field function } \\ H_{n}(x) & \text { Hermite polynomial of order } n \\ \mathcal{H} & \text { Hermitian kernel } \\ h(v, u) & \text { Linear system spatial impulse function } \\ \mathcal{I} & \text { Integrated intensity } \\ \mathcal{I}_{0} & \text { Integrated intensity of the transmitted field } \\ i(x) & \text { Field intensity } \\ j & =\sqrt{-1} \\ J\left(r_{1}, r_{2}\right) & \text { Mutual intensity } \\ k & \text { Optical wave number } \\ l_{0} & \text { Inner scale of turbulence } \\ L_{0} & \text { Outer scale of turbulence }\end{array}$




\begin{tabular}{|c|c|}
\hline$L_{p}^{m}(x)$ & Laguerre polynomial \\
\hline$n$ & Index of refraction \\
\hline$n_{s}$ & Fluctuation of the index of refraction \\
\hline $\mathcal{R}$ & Receiver region \\
\hline$r$ & $=\|\vec{r}\|$ \\
\hline$\vec{r}$ & Position vector $=(x, y, z)$ \\
\hline$r_{0}$ & Fried parameter \\
\hline$T$ & Temperature \\
\hline$t$ & Time \\
\hline$\vec{u}$ & Position vector in transmitter plane $=(x, y)$ \\
\hline$\vec{v}$ & Position vector in receiver plane $=(x, y)$ \\
\hline$w(v)$ & Weighting function at receiver \\
\hline$x$ & Distance in the plane transverse to direction of propagation \\
\hline$y$ & Distance in the plane transverse to direction of propagation \\
\hline$z$ & Distance in direction of propagation \\
\hline$\alpha$ & Coefficients of the coherent modes \\
\hline$\chi$ & Log amplitude \\
\hline$\Gamma_{2}$ & Seconde moment of the field \\
\hline$\Gamma_{4}$ & Fourth moment of the field \\
\hline$\gamma$ & Complex degree of coherence \\
\hline$\kappa$ & Wave number of index-of-refraction fluctuations \\
\hline$\lambda$ & Wave length \\
\hline$\mu$ & Mean of the integrated intensity \\
\hline$\nu$ & Spacial frequency \\
\hline$\omega$ & Radian frequency $=2 \pi f$ \\
\hline$\Phi_{n}$ & Spectrum of the refractive index fluctuations \\
\hline$\phi$ & Phase perturbation of wave field \\
\hline$\psi$ & Complex phase perturbation or coherent modes \\
\hline$\rho_{0}$ & Transverse coherence distance \\
\hline
\end{tabular}


Time shift $=t_{2}-t_{1}$

Propagation angle 


\section{Bibliography}

[1] L. C. Andrews and R. L. Phillips. Laser Beam Propagation Through Random Media. SPIE, 1998.

[2] L. C. Andrews, R. L. Phillips, and C. Y. Hopen. Laser Beam Scintillation with Applications. SPIE, 2001.

[3] Larry C. Andrews. Aperture-averaging factor for optical scintillations of plane and spherical waves in the atmosphere. J. Opt. Soc. Am. A, $9(4): 597-600,1992$.

[4] H. W. Badcock. The possibility of compensating astronomical seeing. Publ. Astron. Soc. Pac., 65:229-236, 1953.

[5] H. W. Badcock. Adaptive optics revisited. Science, 249:253-257, 1990.

[6] V. A. Banakh, G. M. Krekov, V. L. Mironov, S. S. Khmelevtsov, and R. Sh. Tsvik. Focused-laser-beam scintillations in the turbulence atmosphere. J. Opt. Soc. Am., 64(4):516-518, 1974.

[7] Robert R. Beland. Propagation through atmospherc optical turbulence, volume IR/EO Handbook 2, chapter 2, pages 157-232. MI and SPIE Engineering Press, 1993. 
[8] M. Beran. Propagation of a finite beam in a random medium. J. Opt. Soc. Am., 60(4):518-521, 1970.

[9] P. Bergmann. Propagation of radiation in a medium with random inhomogeneities. Phys. Rev., 70:486-492, 1946.

[10] Max Born and Emil Wolf. Principles of Optics. Cambridge U. Press, Cambridge, UK, 7 edition, 1999.

[11] W. Brown. Moment equations for waves propagated in random media. J. Opt. Soc. Am., 62:45-54, 1972.

[12] L. A. Chernov. Wave Propagation in a Random Medium. Dover Publications, 1967.

[13] T. Chiba. Spot dancing of the laser beam propagated through the turbulent atmosphere. Appl. Opt., 10(11):2456-2461, 1971.

[14] James H. Churnside. Aperture averaging of optical scintillations in the turbulent atmosphere. Appl. Opt., 30(15):1982-1994, 1991.

[15] W. A. Coles, J. P. Filice, R. G. Frehlich, and M. Yadlowsky. Simulation of wave propagation in three-dimensional random media. Appl. Opt., 34(12):2089-2101, 1995.

[16] R. Courant and D. Hilbert. Methods of Mathematical Physics, volume 1. Interscience Publishers, Inc., New York, 1953.

[17] D. deWolf. Strong irradiance fluctuations in turbulent air ii: Spherical waves. J. Opt. Soc. Am., 63:1249-1253, 1973. 
[18] D. deWolf. Strong irradiance fluctuations in turbulent air: Plane waves. J. Opt. Soc. Am., 63:360-365, 1973.

[19] Federico Dios, Juan Antonio Rubio, Alejandro Rodriguez, and Adolfo Comeron. Scintillation and beam-wander analysis in an optical group station-satelite uplink. Applied Optics, 43(19):3866-3873, Jul 2004.

[20] J. Dunphy and J. Kerr. Scintillation measurements for large integrated path turbulence. J. Opt. Soc. Am., 63(8):981-986, 1973.

[21] R. Elliott, J.kerr, and P. Pincus. Optical propagation in laboratory generated turbulence. Appl. Opt., 18:3315-3323, 1979.

[22] R. Fante. Propagation of electromagnetic waves through a turbulent plasma using transport theory. IEEE Trans. Antennas Propagat., AP21:750-755, Sep 1973.

[23] Ronald. L. Fante. Electromagnetic beam propagation in turbulent media. In Proc. IEEE, volume 63, pages 1669-1692, 1975.

[24] Ronald L. Fante. Electromagnetic beam propagation in turbulent media: An update. In Proc. IEEE, volume 68, pages 1424-1443, 1980.

[25] Ronald L. Fante. Wave propagation in random media: A system approach. In Emil Wolf, editor, Progress in Optics, volume XXII, chapter VI, pages 343-398. North-Holland Publishing Company, Amsterdam, 1985

[26] D. L. Fried. Aperture averaging of scintillation. J. Opt. Soc. Am., 57(2):169-175, 1967. 
[27] R. M. Gagliardi and S. Karp. Optical Communications. John Wiley Sons, Inc, 2 edition, 1995.

[28] G. Gbur and E. Wolf. Spreading of partially coherent beams in random media. J. Opt. Soc. Am. A, 19(8):1592-1598, 2002.

[29] T. Goldring and L. Carlson. Analysis and implementation of nonkolmogorov phase screens appropriate to structured environments. In SPIE proceedings on Nonlinear Optical Beam Manipulation and High Energy Beam Propagation Through the Atmosphere, volume 1060, pages 244-264. SPIE, 1989.

[30] J. Goodman. Statistical Optics. John Wiley, 1985.

[31] J. Goodman. Introduction to Fourier Optics. John Wiley, 1996.

[32] M. Gracheva, A. Gurvich, and M. Kallistrova. Dispersion of strong atmospheric fluctuations in the intensity of laser radiation. Radiophys. Quantum Electron., 13(1):40-42, 1970.

[33] J. Hardy. Adaptive Optics for Astronomical Telescopes. Oxford University Press, 1998.

[34] G. Homstad, J. strohbehn, R. Berger, and J. Heneghan. Apertureaveraging effects for weak scintillations. J. Opt. Soc. Am., 64(2):162$165,1974$.

[35] R. E. Hufnagel. Propagation through atmospheric turbulence / The Infrared Handbook, chapter 6. US Office of Naval Research, Washington D.C., 1978. 
[36] A. Ishimaru. Flucturations of a focused beam wave for atmospheric turbulence probing. In Proc. IEEE, volume 57, pages 407-414. IEEE2, 1969.

[37] A. Ishimaru. Wave Propagation and Scattering in Random Media. Academic Press, 1978.

[38] P. Jacquinot and Mme B. Roizen-Dozzier. Apodisation. In Emil Wolf, editor, Progress in Optics, volume III, chapter II, pages 31-186. NorthHolland Publishing Company, Amsterdam, 1964.

[39] A T Jones and J A McMordie. Thermal blooming of continuous wave laser radiation. J. Phys. D: Appl. Phys, 14:163-172, 1981.

[40] Avinash C. Kak and Malcolm Slaney. Principles of Computerized Tomographic Imaging. SIAM, 2001.

[41] J. B. Keller. Accuracy and validation of the born and rytov approximation. J. Opt. Soc. Am, 59:1003-1004, 1969.

[42] J. Kerr. Experiments on turbulence characteristics and multi-wavelength scintillation phenomena. J. Opt. Soc. Am, 62(9):1040-1051, 1972.

[43] J. Kerr and J. Dunphy. Experimental effects of finite transmitter apertures on scintillations. J. Opt. Soc. Am., 63(1):1-7, 1973.

[44] A. N. Kolmogorov. Local structure of turbulence in an incompressible fluid at very high reynolds numbers. Dokl. Akad. Nauk SSSR, 30(4):2991941. 
[45] A. N. Kolmogorov. Turbulence, Classic Papers on Statistical Theory. Wiley-Interscience, New York, 1961.

[46] Fridedrich Kottler. The elements of radiative transfer. In Emil Wolf, editor, Progress in Optics, volume III, chapter I, pages 3-28. NorthHolland Publishing Company, Amsterdam, 1964.

[47] R. S. Lawrence and J. W. Strohbehn. A survey of clear-air propagation effects relevent to optical communications. In Proc. IEEE, volume 58, page 1523,1970 .

[48] R. K. Luneberg. Mathematical Theory of Optics. University of California Press, Berkeley, CA, 1944.

[49] R. F. Lutomirski and H. T. Yura. Propagation of a finite optical beam in an inhomogeneous medium. Applied Optics, 10(7):1652-1658, 1971.

[50] L. Mandel and E. Wolf. Optical Coherence and Quantum Optics. Cambridge University Press, 1995.

[51] G. Mie. Beiträge zur optik trüber medien, speziell kolloidaler metallösungen. Ann. Phys., 25:377-445, 1908.

[52] J. Mollyneux. Propagation of nth order coherence functions in a random medium. J. Opt. Soc. Am., 61(9):1134-1138, 1971.

[53] J. C. Owens. Optical refractive index of air: Dependence on pressure, temperature and compositions. Appl. Opt., 6:51-59, 1967. 
[54] C. Primmerman and D. Fouche. Thermal blooming compensation: experimental observation of a deformable mirrow system. Applied Optics, 15:990-995, 1976.

[55] Lord Rayleigh. On the light from the sky, its polarization and colour. Philos. Mag., 41:107-120, 274-279, 1871. Reprinted in Scinentific Papers by Lord Rayleigh, Vol. I: 1869-1881, No. 8, Dover, New York, 1964.

[56] Jennifer C. Ricklin and Frederic M. Davidson. Atmospheric turbulence effects on a partially coherent gaussian beam: implications for free-space laser communication. J. Opt. Soc. Am. A, 19(9):1794-1802, 2002.

[57] Jennifer C. Ricklin and Frederic M. Davidson. Atmospheric optical communication with a gaussian schell beam. J. Opt. Soc. Am. A, 20(5):856866, 2003.

[58] Michael C. Roggemann and Byron Welsh. Imaging Through Turbulence. CRC Press, 1996.

[59] Sergei M. Rytov, Yurii A. Kravtsov, and Valeryan I. Tatarskii. Principles of Statistical Radiophysics, volume 1-4. Springer, 1987.

[60] Bahaa E. A. Saleh and Malvin C. Teich. Fundamentals of Photonics. John Wiley \& Sons, Inc, 1991.

[61] R. A. Schmeltzer. Means, variance and covariance for laser beam propagation through a random medium. Q. Appl Math., 24:339-354, 1966.

[62] Timothy J. Schulz. Iterative transform algorithm for the computation of optimal beams. J. Opt. Soc. Am. A, 21(10):1970-1974, 2004. 
[63] Timothy J. Schulz. Optimal beams for propagation through random media. Optical Letters, 30(10):1093-1095, 2005.

[64] J. Shapiro. Reciprocity of the turbulent atmosphere. J. Opt. Soc. Am., 61(4):492-495, 1971.

[65] Tomohiro Shirai, Aristide Dogariu, and Emil Wolf. Mode analysis of spreading of partially coherent beams propagating through atmorspheric turbulence. J. Opt. Soc. Am. A, 20(6):1094-1102, 2003.

[66] Nicholas M. Short. Remote sensing tutorial. http://rst.gsfc.nasa.gov/, 2006 .

[67] A. Siegman. Lasers. University Science Books, 1986.

[68] D. Slepian. Analytic solution of two apodization problems. J. Opt. Soc. Am., 55(9):1110-1115, 1965.

[69] D. Slepian and H. O. Pollak. Prolate spheroidal wave function, fourier analysis and uncertainty - i. Bell Syst. Tech. J., 40(43), 1961.

[70] D. Smith. High power laser propagation: Thermal blooming. Proc. IEEE, 65:1345-1358, 1977.

[71] R. Straubel. Pieter Zeeman. Verhandelingen op 25 Mei 1935 Aangeboden .aan Prof. Dr. P. Zeeman. Martinus Nijhoff, Hague, Netherlands, 1935.

[72] J. W. Strohbehn. Line-of-sight wave propagation through the turbulent atmosphere. In Proceedings of the IEEE, volume 56, pages $1301-1318$, 1986. 
[73] V. I. Tatarski. Waves Propagating in a Turbulent Medium. Dover publications, New York, 1967.

[74] V. I. Tatarski. Light propagation in a medium with random refractive index inhomogeneites in the markov approximation. Sov. Phy. JETP, 29:1133-1138, Dec 1969.

[75] Robert K. Tyson. Principles of Adaptive Optics. Academic Press, 1997.

[76] D Voelz and Kevin Fitzhenry. Pseudo-partially coherente beam for freespace laser communications. 2005.

[77] Mikhail A. Vorontsov and Valeriy Kolosov. Target-in-the-loop beam control: basic considerations for analysis and wave-front sension. $J$. Opt. Soc. Am. A, 22(1):126-141, 2005.

[78] Qingsong Wang and Michael Giles. Coherence reduction using optical fibers. In D. Voelz and J. Ricklin, editors, Free Space Laser Communications $V$, volume 5892. SPIE, 2005.

[79] S. J. Wang, Y. Baykal, and M. A. Plonus. Receiver-aperture averaging effects for the intensity fluctuations of a beam in the turbulent atmorsphere. J. Opt. Soc. Am., 73(6):831-837, 1983.

[80] Hugo Weichel. Laser Beam Propagation in the Atmorsphere. SPIE Press, 1990.

[81] Eric W. Weisstein. Millimeter/Submillimeter Fourier Transform Spectroscopy of Jovian Planet Atmospheres. PhD thesis, California Institute of Technology, Pasadena, California, 1996. 
[82] H. T. Yura. Mutual coherence function of a finite cross section optical beam propagating in a turbulent medium. Appl. Opt., 11(6):1399-1406, 1972. 